\title{
Multicomponent Domino Reaction in the Asymmetric Synthesis of Cyclopentan[c]pyran Core of Iridoid Natural Products
}

\author{
Alejandro Manchado, Victoria Elena Ramos, David Díez@ and Narciso M. Garrido *(1) \\ Dpto. de Química Orgánica, Facultad de Ciencias Químicas, Universidad de Salamanca, Plaza de los Caídos 1-5, \\ 37008 Salamanca, Spain; alex92mc@usal.es (A.M.); velenaramos@yahoo.com (V.E.R.); ddm@usal.es (D.D.) \\ * Correspondence: nmg@usal.es; Tel.: +34-6665-89065; Fax: +34-9232-94574
}

Received: 13 February 2020; Accepted: 8 March 2020; Published: 13 March 2020

\begin{abstract}
The asymmetric synthesis of a compound with the cyclopentan[c]pyran core of iridoid natural products in four steps and $40 \%$ overall yield is reported. Our methodology includes a one-pot tandem domino reaction which provides a trisubstituted cyclopentane with five new completely determined stereocenters, which were determined through 2D homo and heteronuclear NMR and n.O.e. experiments on different compounds specially designed for this purpose, such as a dioxane obtained from a diol. Due to their pharmaceutical properties, including sedative, analgesic, anti-inflammatory, CNS depressor or anti-conceptive effects, this methodology to produce the abovementioned iridoid derivatives, is an interesting strategy in terms of new drug discovery as well as pharmaceutical development.
\end{abstract}

Keywords: chiral lithium amide; asymmetric aza-Michael addition; asymmetric domino reaction; multicomponent reaction; cyclopentan[c]pyran; iridoid; nepetalactone

\section{Introduction}

Iridoids are a very extensive family of secondary metabolites. They are found in both terrestrial and marine flora and fauna [1-3]. Although usually found in their glucoside form, free iridoids as well as secoiridoids are also abundant in Nature. As free iridoids, they are precursors of biologically active alkaloids and several studies have demonstrated their interesting pharmaceutical properties, as hematoprotective or $\mathrm{N}$-oxide inhibitor agents [4-6]. As glycosides, they are commonly found in plants, such as in the genus Nepeta, and, since they are structurally cyclopentane pyran monoterpenoids, they represent a link between terpenes and alkaloids [7]. Recent studies also suggest they are cell proliferation inhibitors, opening an interesting window into cancer treatment $[8,9]$ as well as viral protein P (Vpr) inhibition [10]. Finally, as secoiridoids, they possess lots of biological activities such as antioxidant, anti-inflammatory, or anti-atherogenic properties, among others [11-13]. This family of compounds has also been extensively used in folk medicine plant treatments, all around the globe, as a remedy against coughs, wounds or skin disorders, as well as bitter tonics, antipyretics or sedatives [14,15].

The genus Nepeta, with its bigger diversity located in the Mediterranean area, have demonstrated, in recent studies, that some extracts from different plants possess very interesting therapeutic properties, such as anti-inflammatory or analgesic effects, due to the abundant presence of nepetalactone derivatives [16,17]. This opens a promising researching area, since morphine use, nowadays, is responsible a lot of dependency and deaths. Also, recent studies have demonstrated the high diversity of pharmacological properties of nepetalactone compounds $[8,18,19]$. They show both sedative and analgesic properties, as well as anti-inflammatory and CNS depressor effects, as new studies suggest 
this kind of compound is able to pass through the blood brain barrier [16]. Plus, recent studies have demonstrated nepetalactone derivatives present anticonceptive activity as well as insect repellent ability [19-22].

We have developed an asymmetric synthesis-based route, which enabled us to obtain the $4 \beta, 4 \mathrm{a} \alpha, 7 \alpha, 7 \mathrm{a} \alpha$-dihydronepetalactone analogue 26 (Figure 1,) in very good yield while controlling all chiral centers. This compound is an advanced intermediate towards nepetalactone and iridoid skeletons which have been demonstrated to possess very interesting analgesic properties. Some examples of nepetalactones and iridoids accessible using our methodology are illustrated in Figure 1, where the relevance of the recently isolated secoiridoid I from Fraxinus americana L.[23] with an identical cyclopentane core can be mentioned, highlighting the importance of $\mathbf{2 6}$ for its synthesis [23-25].

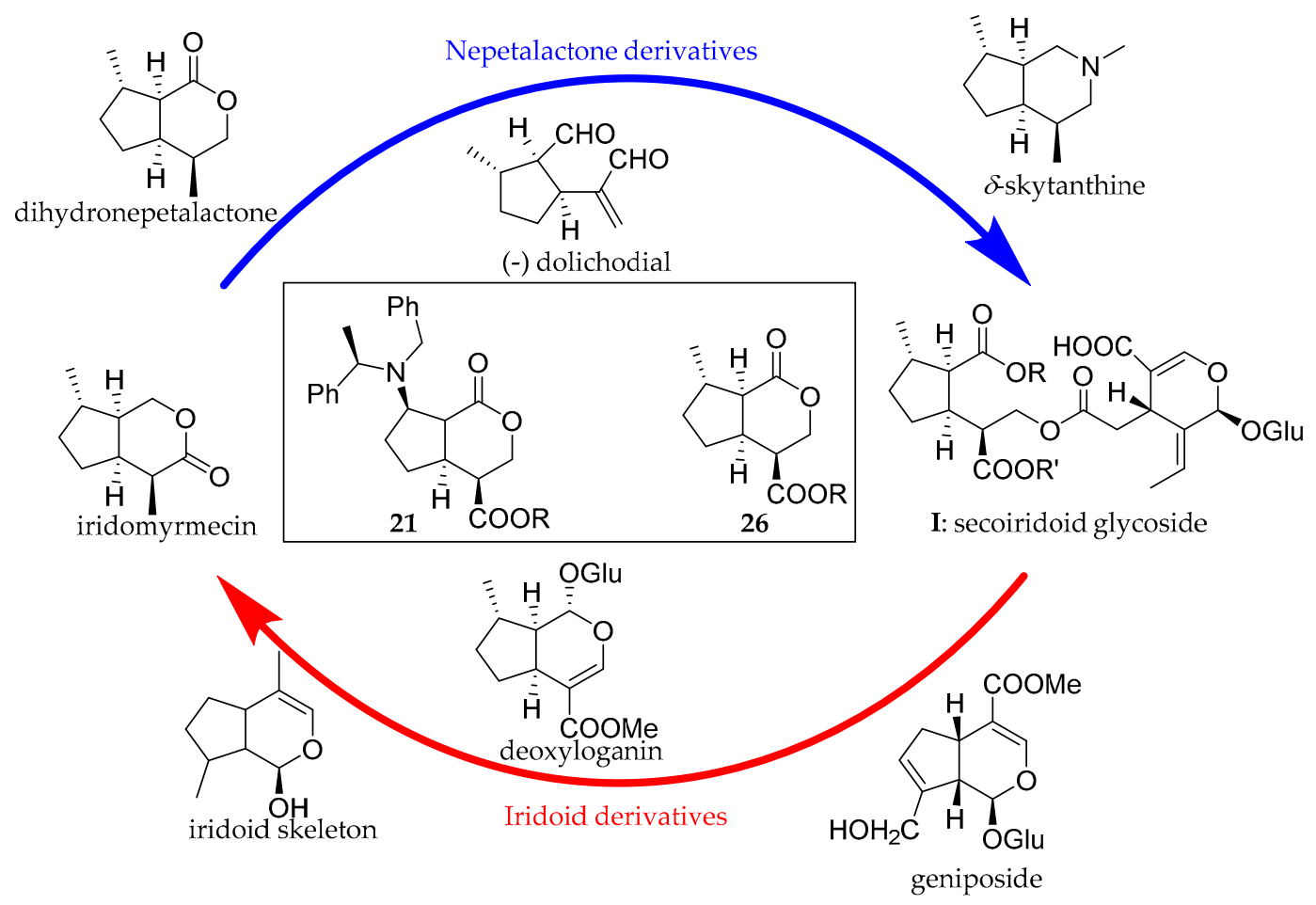

Figure 1. Some main nepetalactone and iridoid skeletons.

Davies et al. have published a comprehensive review concerning the development, scope and applications of the conjugate additions of enantiomerically pure lithium amides (which act as chiral ammonia equivalents) in 2005, and an update covering 2005-2011 was published in 2012. A further update was recently published in 2017 [26-28], dealing with all the characteristics of the asymmetric addition, and recently, we have published a chapter describing methods for the synthesis of lithium amides and their applications in C-N and C-C bond formation reactions, including stereoselective transformations [29].

We have demonstrated the use of chiral lithium ( $\alpha$-methylbenzyl)benzylamide $(R)$ - or $(S)-\mathbf{1})$ in different domino reactions. We first published that a chiral lithium amide could initiate an asymmetric conjugate addition cyclization of nona-2,7-diendioate to generate chiral cyclohexane derivatives (Scheme 1, II) [30-33], and applied it to the synthesis of $(1 R, 5 R, 9 R)$-2-azabicyclo-[3.3.1]nonane-9-carboxylic acid (morphanic acid), with a morphan scaffold [34], which was used in the synthesis of a new class of opioid receptor ligands [32]. In the same way, when octa-2,6-diendioate was used we stereoselectively obtained the 2-amino-5-carboxy-methyl-cyclopentane-1-carboxylate skeleton (Scheme 1, III) [35-37], and applied it to the synthesis of $(R)$ and $(S)$-methyl(-methoxycarbonylcyclopent-2-enyl)acetate IV and $(R)$ - and (S)-2-(2-hydroxymethyl-cyclopent-2-enyl)ethanol, useful homochiral synthons for monoterpenes [35] and to the asymmetric synthesis of all the stereoisomers of 2-amino-5- 
carboxymethyl-cyclopentane-1-carboxylic acid [36]. We have later shown a novel domino reaction: allylic acetate rearrangement stereoselective Ireland-Claisen rearrangement and asymmetric Michael addition [38-41]. A protocol starting from Baylis-Hillman adducts using chiral lithium amide $(R)-\mathbf{1}$ to afford $\delta$-aminoacids, which can be transformed into piperidines [40] Furthermore we get ready access to phenethylamines from ( $N$ - $\alpha$-methylbenzyl)- $N$-benzyl $\beta$-aminoacids obtained by Michael addition of $(R)-1$ to $\alpha$ - $\beta$-unsaturated ester, by domino reaction initiated in a Barton decarboxylation followed by a 1,4-phenyl radical rearrangement (1,4-PhRR) [42].

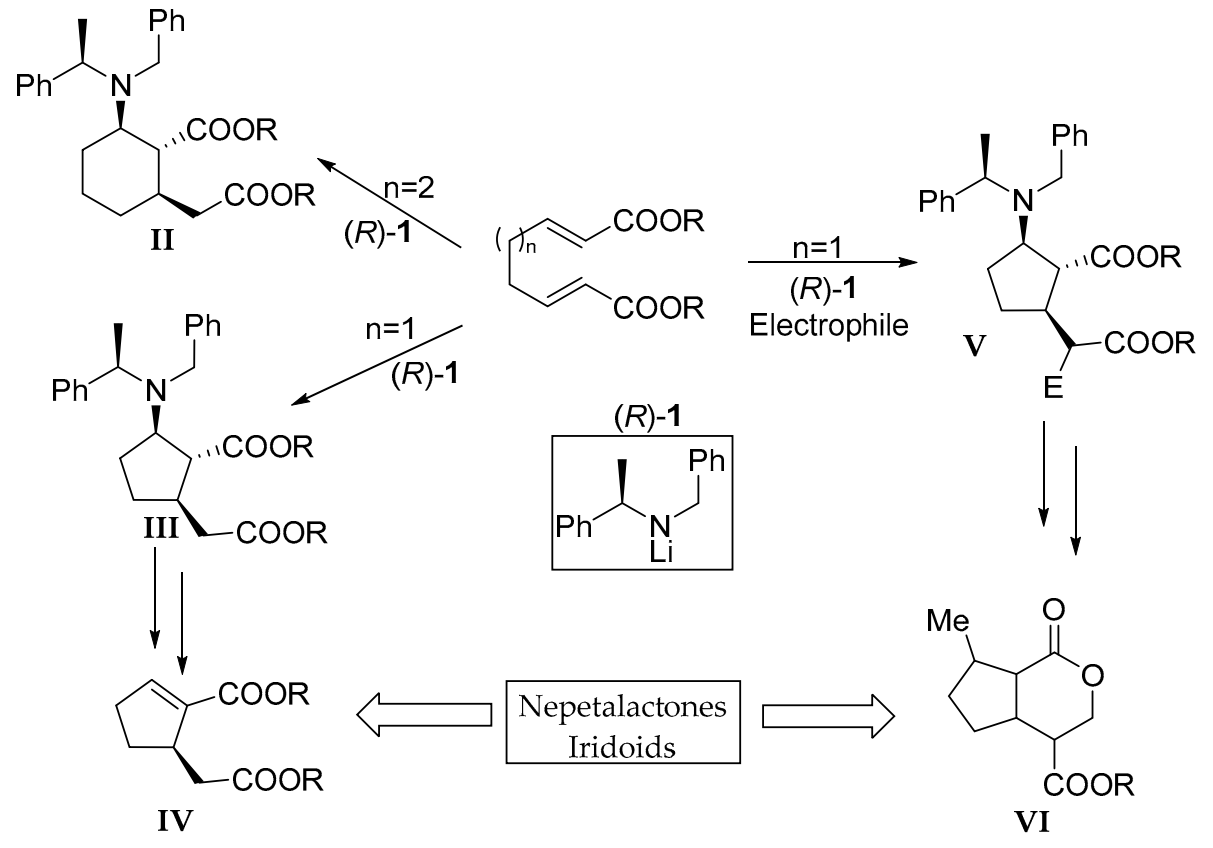

Scheme 1. Previous and current studies of domino reactions.

Herein, as shown in retrosynthetic Scheme 2, following the aforementioned domino reaction access to cyclopentane derivatives, now in a three component version, we report the synthesis of highly functionalized cyclopentanes VIII with total stereocontrol of the four new stereocenters generated in one-pot and their application via cyclization (VII) to the synthesis of important derivatives from the point of view of their pharmaceutical activities, such as nepetalactones and iridoids.

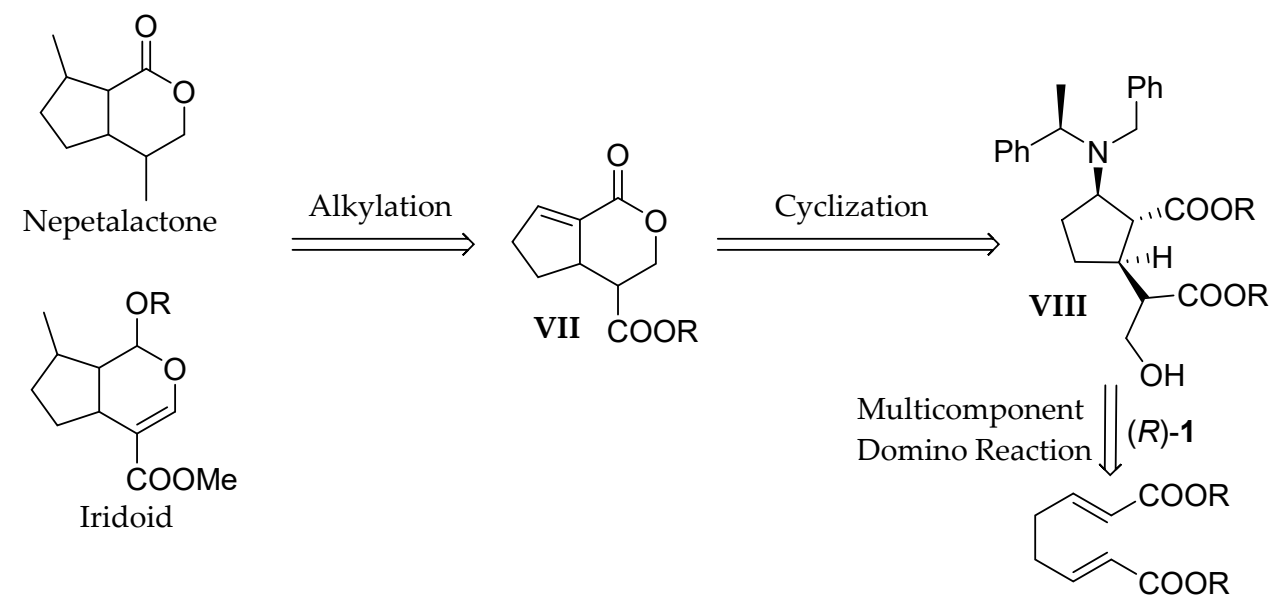

Scheme 2. Retrosyntheses of nepetalactones and iridoids from octadiendioate. 


\section{Results and Discussion}

\subsection{Domino and Tandem Reaction with Benzaldehyde}

Cyclopentane derivative III has been synthesized, as already mentioned, by adding (R)-1 to 2 without a subsequent electrophile addition [35-37]. We wanted to introduce an additional carbon atom in the $\alpha$-position of the alkoxycarbonylmethyl group, so an electrophile was necessary after performing the abovementioned domino reaction in a tandem multicomponent reaction protocol. Thus, we decided initially to use benzaldehyde as electrophile so the reaction scope, as well as the stereochemistry of the two new generated stereocenters, could be studied.

When the addition of $(R)-\mathbf{1}(1.6 \mathrm{eq})$ to di-3-pentyl octa-2,6-diendioate is performed at $-78^{\circ} \mathrm{C}$ and then after one hour, benzaldehyde is added and the reaction allowed to reach room temperature, a mixture from which alcohol 3 (23\%) and the C-1"' epimer 4 (45\%) (Scheme 3b, showing the numbering of these derivatives) are isolated by column chromatography is obtained. The 3-pentyl ester was chosen as it largely prevents the 1,2 addition reactions of the lithium amide to the ester (methyl) group, leading to the corresponding amide and, at the same time, it is easy to hydrolyze under basic conditions. In addition, with the pentyl ester in the domino reaction to obtain the cyclic compound III, we have observed an increase in d.r.: 92:8 vs. 91:9 when using the methyl ester [36].
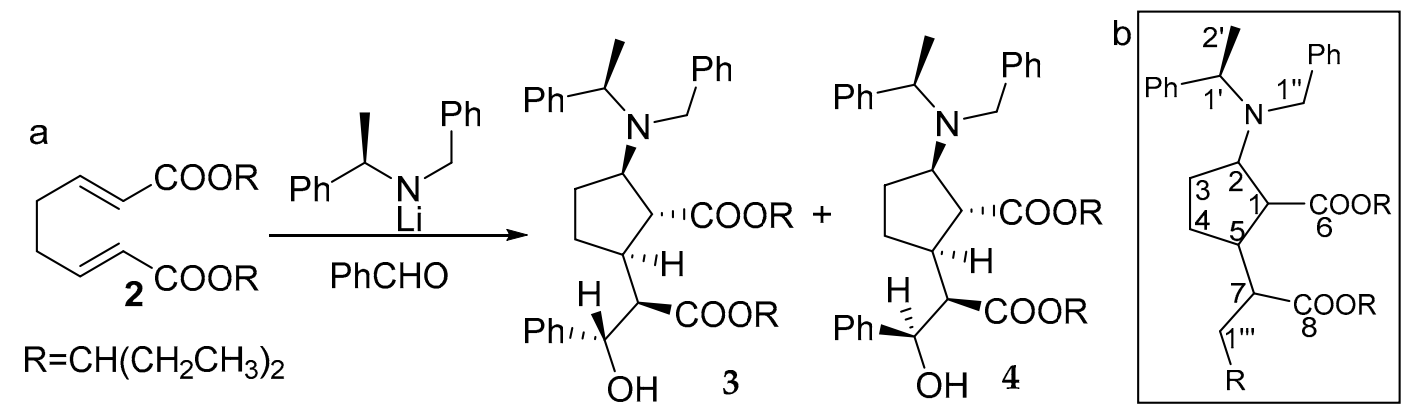

Scheme 3. (a) Multicomponent domino reaction towards 3 and 4. (b) Numbering used for these compounds.

The results of the ${ }^{1} \mathrm{H}^{-13} \mathrm{C}$ heteronuclear correlation experiments at one and several bonds (normal and long range HMQC and HMBC), shown in Table 1 and in Supplementary Materials, allow to corroborate their structure and the full assignment of the ${ }^{1} \mathrm{H}$ - and ${ }^{13} \mathrm{C}-\mathrm{NMR}$ data.

The observed n.O.e (Figure 2a) between $\mathrm{H} 2$ and $\mathrm{H} 5$ for these compounds and the coupling constant in 4 for $\mathrm{H} 1$ at $3.23 \mathrm{ppm}(\mathrm{dd}, J=10.1$ and $9.3 \mathrm{~Hz})$ confirm the predicted trans, transtrisubstituted cyclopentane ring. Compounds 3 and 4 show very similar ${ }^{1} \mathrm{H}$ and ${ }^{13} \mathrm{C}$ data according to the $\mathrm{C}^{\prime \prime \prime}$ different configuration, but full stereochemical characterization was possible by chemical transformations and spectroscopic analysis, as it will be detailed later.

Once the stereochemistry of all sterocenters of $\mathbf{3}$ and $\mathbf{4}$ was known (vide infra), it was possible to explain the experimental observations, such as the $J$ of the hydroxyl hydrogen at $3.92 \mathrm{ppm}(\mathrm{d}, J=9.1)$ and $\mathrm{H}^{\prime \prime \prime}$ at $4.87 \mathrm{ppm}(\mathrm{dd}, J=9.1$ and $3.7 \mathrm{~Hz})$ in 3 and $3.04 \mathrm{ppm}(\mathrm{d}, J=3.5 \mathrm{~Hz}) ; 4.64 \mathrm{ppm}(\mathrm{dd}, J=10.0$ and $3.5 \mathrm{~Hz}$ ) respectively for 4 . 
Table 1. One bond and long-range $2 \mathrm{D}^{1} \mathrm{H}^{13} \mathrm{C}$ correlation for compounds 4,5 and 6 .

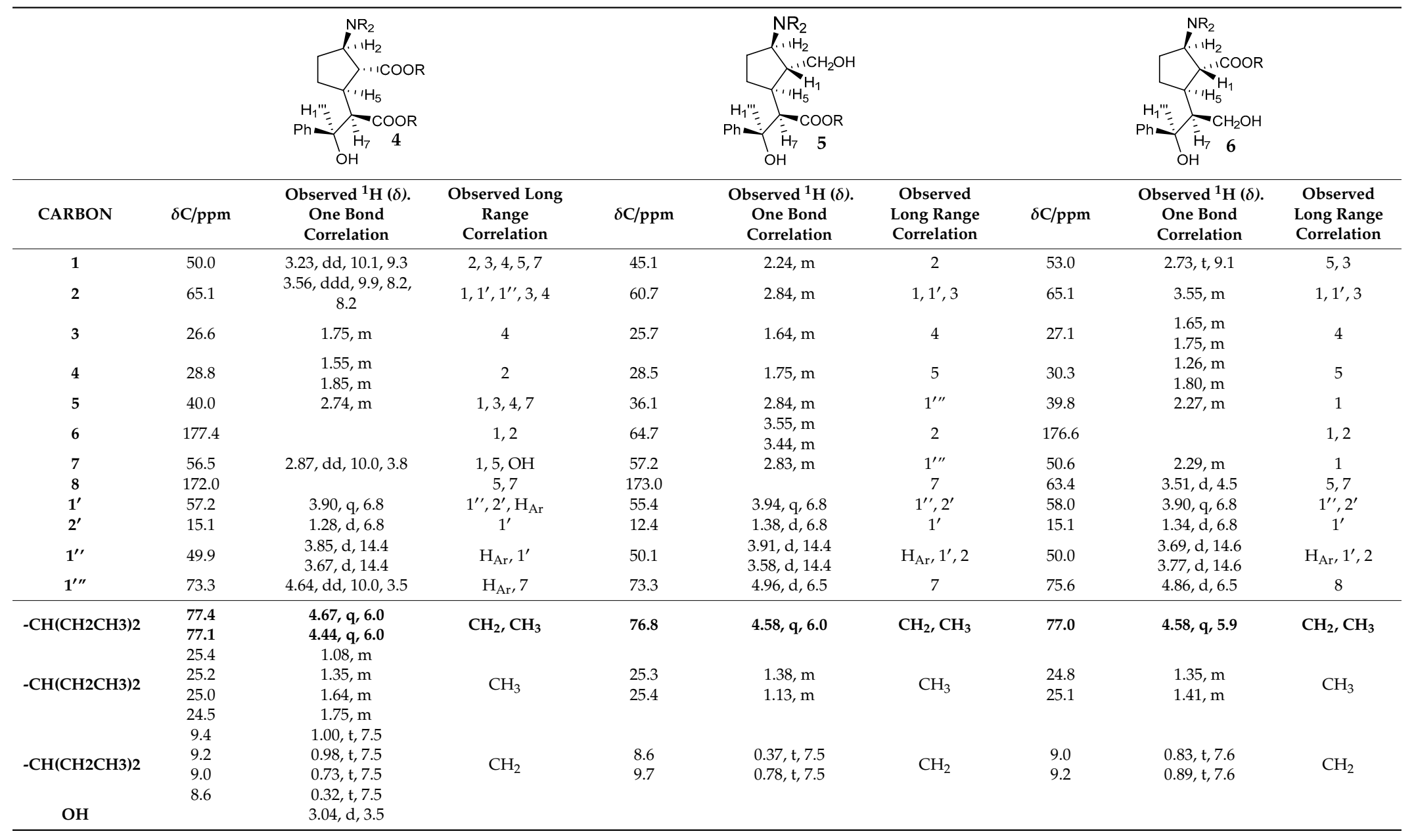


a

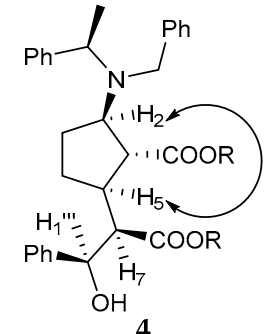

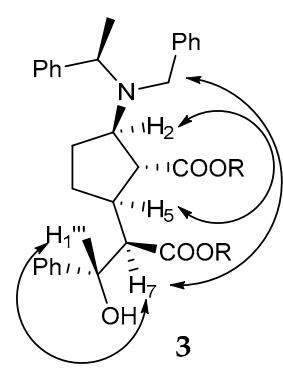

$\mathrm{b}$

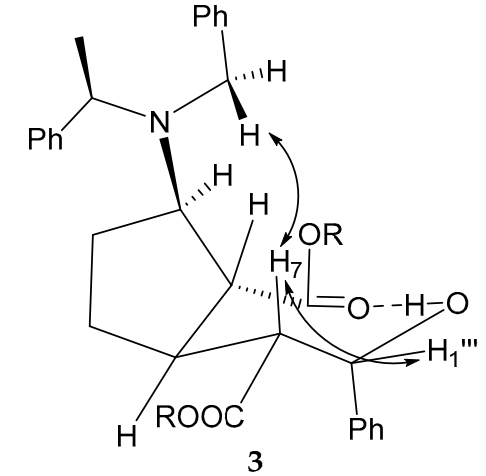

Figure 2. (a) ${ }^{1} \mathrm{H} N M R$ stereochemical analysis of major and minor diastereoisomers 4 and 3. (b) Proposed intramolecular H-bond for 3.

The n.O.e with $\mathrm{H} 1$ by saturation of $\mathrm{H7}$ and a hydrogen of the benzyl C2' in 3 allowed us to conclude the existence of a hydrogen bridge in a cyclooctane system formed between the hydroxyl group and the C6 carbonyl as shown in Figure 2b. These observations could be used for the stereochemical determination of the centers generated in the aldol condensations of these systems.

Then, different reductions were performed as shown in Scheme 4 . When 4 was treated both with $\mathrm{LiAlH}_{4}$ (2 eq.) at $0{ }^{\circ} \mathrm{C}$ and with DIBALH ( 3 eq.) at $-78^{\circ} \mathrm{C} ; 5,6$ and the triol 7 were obtained. HMQC and HMBC studies (Table 1) show correlation between H7 and C-8 ester in diol 5, and correlations between C-6 and $\mathrm{H} 1$ and $\mathrm{H} 2$ in $\mathbf{6}$. Thus, suggesting that the formation of the proposed hydrogen bond (Figure 2b) is favorable at low temperatures, because of both entropic contributions and the Boltzmann distribution, and, therefore, favors the reduction of the C6 ester and, additionally, because of the C-8 ester within this structure is blocked by the phenyl and cyclopentyl groups, bringing on the formation of 5 .
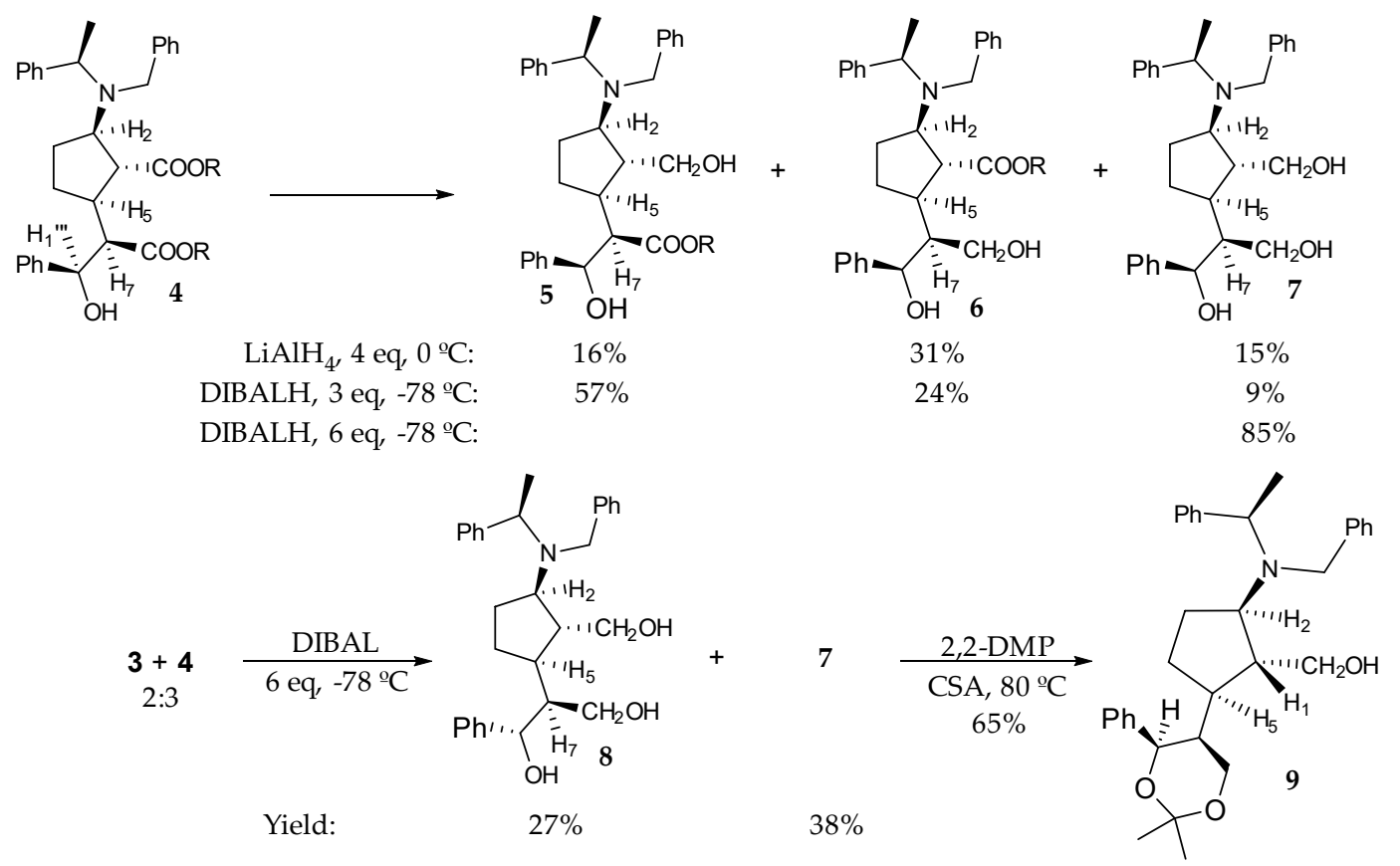

Scheme 4. Reduction reactions of 3 and 4 epimers and dioxane 9 formation.

When the reduction of the mixture $3+4$ (2:3 ratio) was performed with excess DIBALH, the triols 7 and 8 were obtained accordingly, and, under these conditions, reduction of 4 afforded 7 in $85 \%$ isolated yield that was converted in dioxane derivative 9 (61\%) under standard condition when it was treated with dimethoxypropane (Scheme 4). 
Homonuclear COSY and n.O.e. (Figure 3) experiments allowed to determine the stereochemistry in the newly generated centers within the dioxane ring (see Supplementary Materials). Coupling constants $J=12.1 \mathrm{~Hz}$ for $\mathrm{H} 8 \beta$ and $\mathrm{H} 8 \alpha$ and 0 and $2.9 \mathrm{~Hz}$, respectively, for $\mathrm{H} 7$, therefore indicating an equatorial disposition for these protons ( $\mathrm{H} 7$ and $\mathrm{H} 8 \alpha$ ). The most relevant n.O.es: H1-H7, H7-H1'", $\mathrm{H} 1^{\prime \prime \prime}-\mathrm{H} 8 \beta, \mathrm{H} 1^{\prime \prime \prime}-\mathrm{Me} \beta, \mathrm{H} 8 \beta-\mathrm{Me} \beta$ and $\mathrm{H} 7-\mathrm{H} 8 \beta$, indicate a cis arrangement for $\mathrm{H} 7, \mathrm{H} 1^{\prime \prime}, \mathrm{H} 8 \beta$ and $\mathrm{Me} \beta$ (1.49 ppm), thus, fixing all stereocenters for 9 as: $\left(1 R, 2 R, 5 S, 7 R, 1^{\prime} R, 1^{\prime \prime \prime} R\right)$ and the same remains for compounds 7 and 4 .

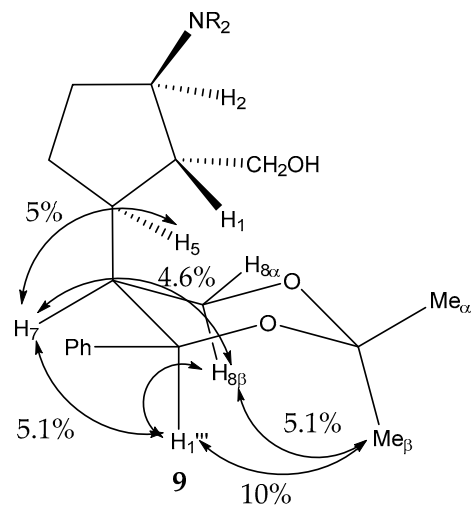

Figure 3. Significant n.O.e correlations observed for $\mathbf{9}$.

As 3 (minor adduct) was presumed to be $\mathrm{C}^{\prime \prime \prime}$ epimer of 4, both the mixture and each compound separately was oxidized with TPAP, always providing the ketone $\mathbf{1 0}$ quantitatively, as shown in Scheme 5 , so the configuration in 3 was established as $\left(1 R, 2 R, 5 S, 7 R, 1^{\prime} R, 1^{\prime \prime \prime} S\right)$. When a mixture of 3 and 4 was oxidized with PDC the diketone 11 was obtained together with 10, due to further oxidation of the benzylic carbon.

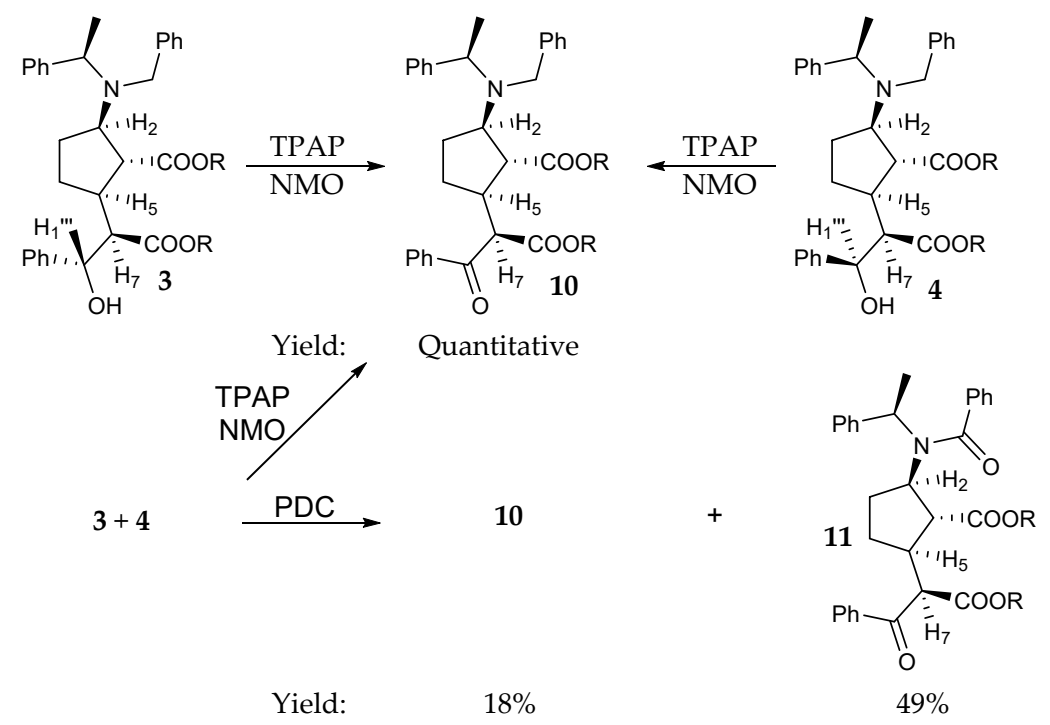

Scheme 5. Oxidation reactions of 3 and 4.

Thus, in summary, we have corroborated by n.O.e experiments the stereochemistry of the three stereocenters of the cyclopentane (achieved by, and related to, the auxiliar chiral (R)-1), that we have already described and settled by $x$-ray crystallography [36]). Likewise, the stereochemistry of the two new stereocenters generated in the subsequent aldol reaction were established by n.O.e experiments on a dioxane derivative of the major diastereoisomer. The other diastereoisomer was the $\mathrm{C} 1^{\prime \prime}$ " epimer of the previous one, since the same compound was obtained by oxidation of this center in both epimers. 


\subsection{Proposed Mechanism}

We have reported an exhaustive mechanistic revision [43] of the originally proposed mechanism [44], developing a quantum mechanics/molecular mechanics protocol for the asymmetric aza-Michael reaction of homochiral lithium benzylamides to $\alpha$ - $\beta$-unsaturated esters resulting in a Z-enolate prior to electrophilic quenching [45].

The second Michael addition in the domino reaction gives rise to the living Z-enolate (IX, Scheme 6) with the Si face accessible for the electrophile. Two approaches are possible for the incoming benzaldehyde: like, throughout its Si face $(\mathbf{X})$ or unlike, Re face of the aldehyde (XI), a 1:2 ratio for 3/4 is observed, probably, due to the contrast of unfavourable axial position of the phenyl group in $\mathbf{X}$, over steric impediment of the cycle substituents in XI. This ratio changes with the size of the aldehyde (vide infra).

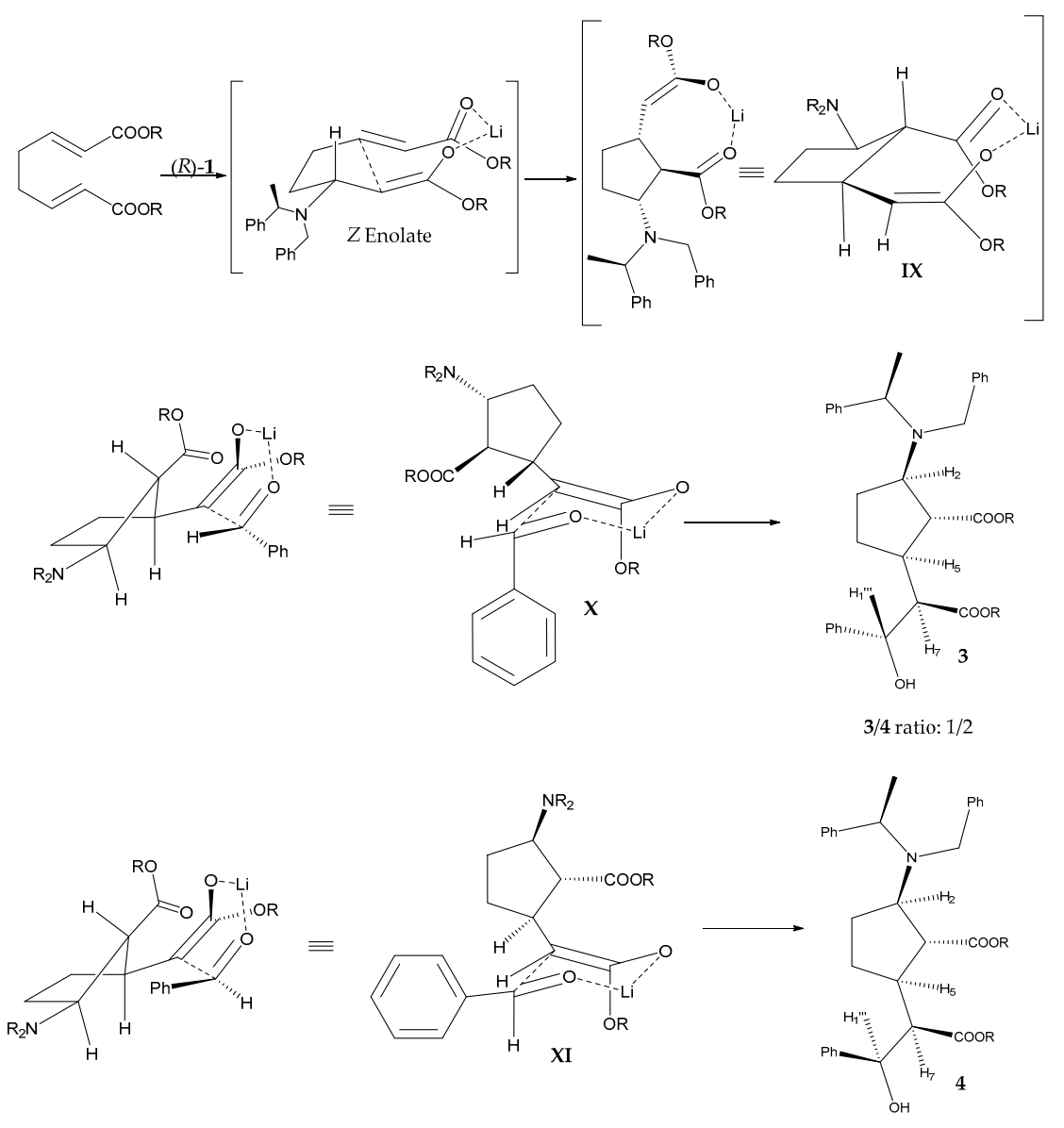

Scheme 6. Mechanistic explanation of the different TS toward 3 and 4 (1:2 ratio).

\subsection{Reaction Scope}

The next step was to react new electrophiles such as different aldehydes, ketones, epoxide and chloroformate to explore the reaction scope. Formaldehyde is an interesting substrate in regards to this methodology due to the possibility of its applications to the synthesis of iridoid natural products.

Results are shown in the following table.

When using cinnamaldehyde as electrophile (Table 2, entry 2), 12 (30\%) and 13 (27\%) were obtained. The NMR signals, taking into account the additional double bond, are similar to those of $\mathbf{3}$ and $\mathbf{4}$, and, based on the spectroscopic considerations established above, especially the hydrogen bridge bond, allow us to establish the stereochemistry of these compounds, the hydroxyl proton in 12 at 3.33 ppm (d, $J=10 \mathrm{~Hz})$ and in $\mathbf{1 3}$ at $2.85 \mathrm{ppm}(\mathrm{d}, J=2.5 \mathrm{~Hz})$ accordingly. In this case the epimer ratio is close to 1:1, in accordance to the proposed mechanism (Scheme 6) due to increased interaction in TS XI. 
Table 2. Electrophile additions.

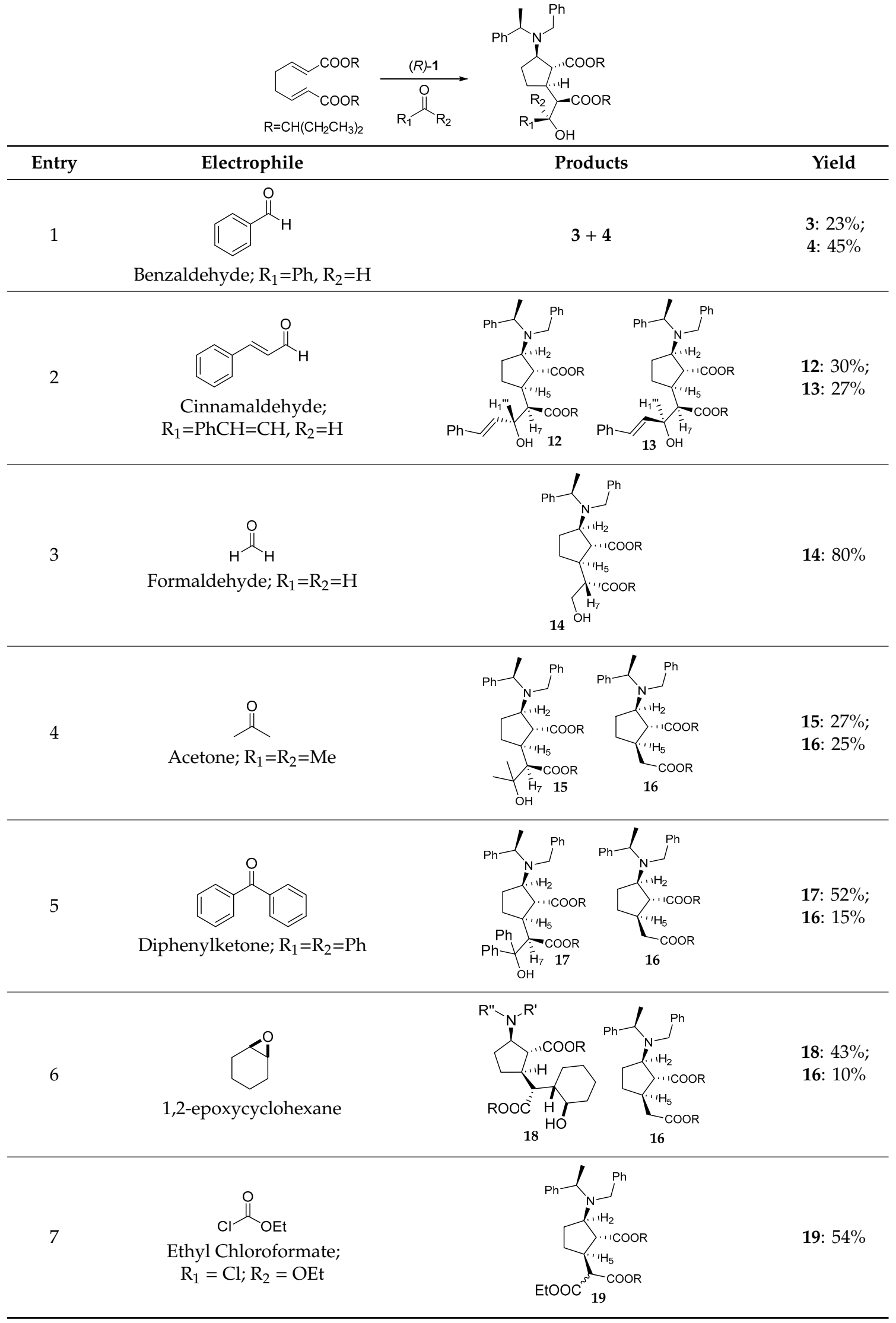


When formaldehyde was used (entry 3), 14 (80\%) was the only compound isolated after column chromatography. Now the C6 configuration is $S$, contrary to previous one, as determined in subsequent derivatives (vide infra). Due to the small size of the formaldehyde molecule, it approaches the $R e$ face probably in a tricoordinate Li TS within IX, producing $\mathbf{1 4 .}$

To explore the reaction scope acetone and diphenylketone (entry 4 and 5) were used, giving rise to the multicomponent adducts $15(27 \%)$ and $17(52 \%)$ respectively, together with the reported [36] domino adduct 16 in 25\% and 15\% respectively, due to the lower reactivity of ketones.

Similarly, $18(43 \%)$ and $16(10 \%)$ were obtained when the reaction was performed with 1,2-epoxycyclohexane as electrophile (entry 6).

Finally, when ethyl chloroformate was used as electrophile, the mixture of epimers in C7 19 (54\%) was obtained as the only isolable compounds, in this case ${ }^{1} \mathrm{H}$ - and ${ }^{13} \mathrm{C}-\mathrm{NMR}$ spectra showed signals corresponding to the mixture. Nevertheless, this could be an interesting triorthogonal derivative to achieve the objectives of the project.

The results obtained indicate that this is an effective methodology capable of supporting the addition of different electrophiles, which allows the incorporation of different potential functionalities in the synthesis of interesting organic molecules and natural products.

\subsection{Application to the Synthesis of the Iridoid Natural Product Core}

It was shown in the retrosynthetic Scheme 2 that iridoid natural products are available from intermediate VII with cyclopentan[c]pyran skeleton, which is available from intermediate VIII, which is the one obtained in the domino reaction and subsequent tandem addition of formaldehyde (compound 14). Key steps towards achieving the objective are: cyclization reaction and substitution of the amine with a methyl group. The first approach was to try cyclization in an acidic medium $(p \mathrm{TsOH})$ but little transformation (5\%) was observed, then, when basic conditions were used, either with $\mathrm{NaOMe}$ or $\mathrm{NaH}$, the dehydration product 20 was obtained quantitatively (Scheme 7), which is an interesting synthon within this methodology. As from our experience the lone pair electrons in amine group prevents reactivity in acidic media, we treated the solution with $\mathrm{HCl}(\mathrm{g})$ prior to the $p \mathrm{TsOH}$ acid addition to obtain in $30 \%$ yield the lactone 21, the precursor of iridomyrmecin (Figure 1). COSY 2D correlation experiments and significant n.O.es (Figure 4) have allowed us to establish both the absolute stereochemistry and the conformation of the molecule. Relevant n.O.es are $\mathrm{H} 5$ with $\mathrm{H} 9$ and $\mathrm{H} 4$ and this last one with $\mathrm{H} 3 \alpha$, showing that these four hydrogens are cis. Also, the $\mathrm{H} 9$ with $\mathrm{H} 3 \beta$ n.O.e is due to a boat conformation for the $\delta$-lactone with the ester group equatorial and $\mathrm{H} 4$ axial (3.08 ppm, ddd, $J=9.8,9.8$ and $7.5 \mathrm{~Hz}$ ), which explains its coupling constants and those of $\mathrm{H} 3 \alpha$ and $\mathrm{H} 3 \beta$. The $\left(4 S, 5 S, 8 R, 9 R, 1^{\prime} R\right)$ stereochemistry assigned for $\mathbf{2 1}$ matches those from $\mathbf{1 4}$.

Taking into account that the lone pair electrons in the amine complicate the cyclization reaction, the stereospecific syn concerted elimination reaction of Cope was tried first, so when 14 was treated with $m$ CPBA, compound 22 was obtained in $82 \%$ isolated yield. When the reaction was carried out directly from the diunsaturated diester $\mathbf{2}$ and after the addition of the $(R)-\mathbf{1}$ amide and formaldehyde, and the reaction crude mixture was treated directly with $m \mathrm{CPBA}, 22$ and $\mathbf{2 3}$ were separated by column chromatography with $69 \%$ and $6 \%$ yield, respectively. Then, cyclization of each derivative was performed with $p \mathrm{TsOH}$, and 24 and 25 were obtained in $77 \%$ and $65 \%$ yield respectively. The observed n.O.e (Figure 4) sets the stereochemistry for these compounds, which are C4 epimers [36] and therefore corroborates those deduced from adduct 14.

Finally, the methylation of $\mathbf{2 5}$ was performed with $\mathrm{Me}_{2} \mathrm{CuLi}$, so 26 was obtained stereoselectively in $80 \%$ yield [46]. The shift of the methyl group at $1.20 \mathrm{ppm}$ is consistent with the one described for mitsugashiwalactone [47] at $1.18 \mathrm{ppm}$, which presents this stereochemistry, compared to onikalactone [48] at $0.99 \mathrm{ppm}$ with the opposite and it is the compound synthesized by Tanahashi et al. which proves the stereochemistry of natural secoiridoid glucoside from Fraxinus americana L. (Figure 1: I) [23]. 


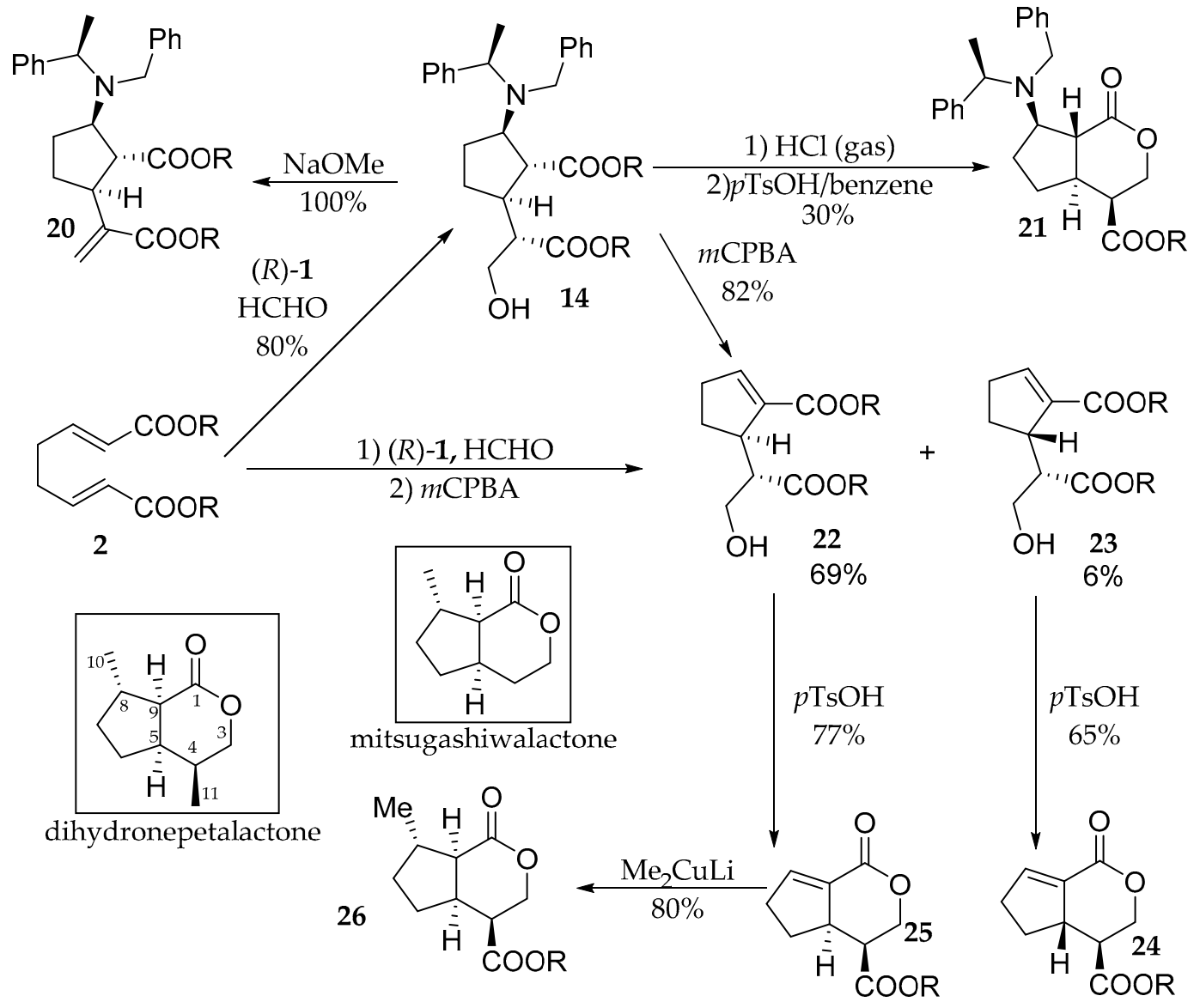

Scheme 7. Synthesis of iridoid derivative 26.
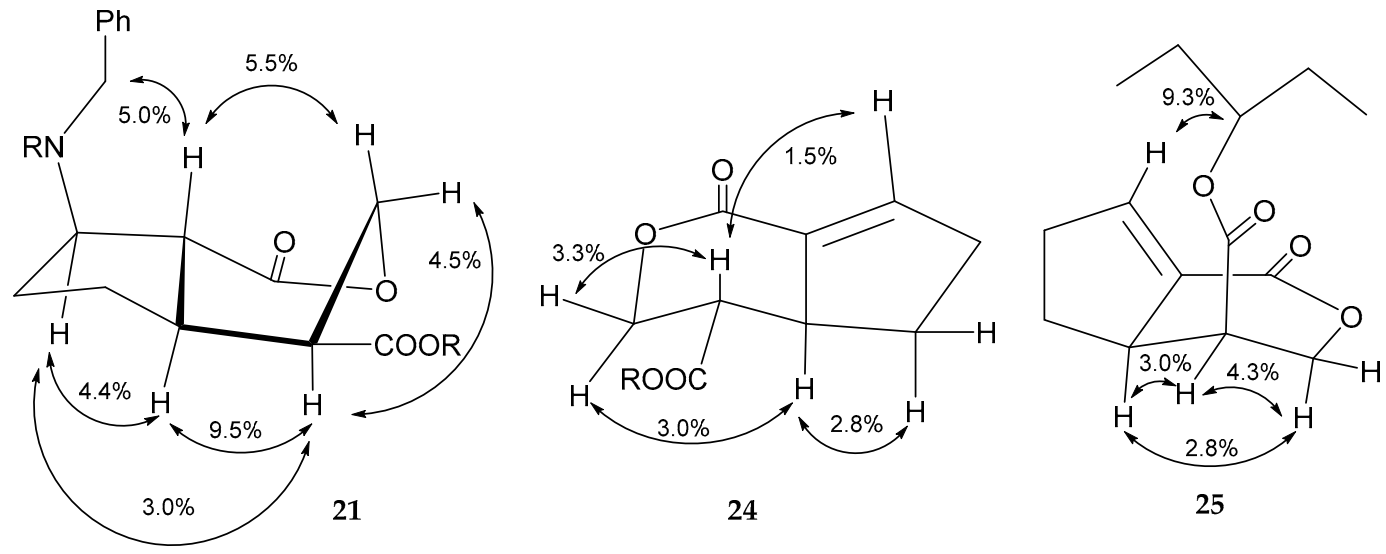

Figure 4. Observed n.O.es for compounds 21, 24 and 25.

\section{Conclusions}

In summary, the total asymmetric synthesis of the iridoid 26, with a methylcyclopentan[c]pyran skeleton, has been carried out in four reaction steps from the affordable octadiendioate 2 and chiral lithium amide $(R)-\mathbf{1}$ and with an overall yield of $40 \%$. This compound is a very advanced analogue of natural products such as: dihydronepetalactone, deoxyloganin or mitsugashiwalactone (by decarboxylation) [42] and it is identical to the cyclopentane system of a new secoiridoid isolated from Fraxinus americana L., so a very effective asymmetric synthesis methodology of iridoidal natural product is developed, providing access to a great diversity of these derivatives. The key step of the synthesis is the initial multicomponent domino reaction, where six sterocenters are developed in 
one pot, the initial four in the domino reaction of Michael addition and intramolecular cyclization and the subsequent two by aldol condensation. Importantly, the analogue series of reactions using the enantiomer of lithium amide $(S)-\mathbf{1}$ in the domino reaction step will allow simple access to the corresponding enantiomers of the aforementioned compounds. Spectroscopic analysis, including homo and heteronuclear two-dimensional correlation experiments have allowed full data assignment and n.O.e and ROESY experiments were performed to determine stereochemistry, especially in derivative products such as dioxolane $\mathbf{9}$, or in the case of formaldehyde as electrophile by obtaining the $\delta$-lactones: 21, 24 and 25. A mechanistic proposal for the reaction course has been postulated and the application to the synthesis of such important natural derivatives as: iridomyrmecin, mitsugashiwalactone and dihydronepetalactone, is underway in our laboratory.

\section{Materials and Methods}

\subsection{General Information}

Nuclear Magnetic Resonance: Both proton NMR $\left({ }^{1} \mathrm{H}-\mathrm{NMR}\right)$ and carbon NMR $\left({ }^{13} \mathrm{C}-\mathrm{NMR}\right)$, as well as 2D homo and heteronuclear experiments, were recorded in deuterated solvents, on spectrometers working on $200 \mathrm{MHz}$ or $400 \mathrm{MHZ}$ for proton and $50 \mathrm{MHz}$ or $100 \mathrm{MHz}$ for carbon, and are shown in the Supplementary Materials. Data shown below is represented as follows: chemical shift, multiplicity, coupling constant, integral and assignment. -Mass Spectroscopy: Mass spectra was recorded using Atmospheric Pressure Chemical Ionization (APCI).

Rotatory Power: Rotatory power data was recorded using $\mathrm{CHCl}_{3}$ as solvent and sodium $\mathrm{D}$ line as polarized light ray.

Infrared Spectrometry: IR data was recorded using liquid IR spectrometer and $\mathrm{NaCl}$ crystal as supporting material.

Column chromatography: Silica column chromatography was performed using silica gel $60 \mathrm{~A}$ (0.060-0.200 mm).

\subsection{General Procedure for the Synthesis of Compounds 3-11}

n-Buli in THF (1.6 M, $1.0 \mathrm{~mL}, 1.60 \mathrm{mmol})$ was added under Ar atmosphere and at $-78{ }^{\circ} \mathrm{C}$ to a solution of $(R)-1,(357 \mathrm{mg}, 1.69 \mathrm{mmol})$ in THF $(7.0 \mathrm{~mL})$. After $50 \mathrm{~min}$ of reaction, a solution of 2 (238 $\mathrm{mg}, 0.77 \mathrm{mmol})$ in THF $(2.5 \mathrm{~mL})$ was added. After $1 \mathrm{~h}$, PhCHO $(2.7 \mathrm{~mL})$ was added and the reaction mixture was stirred until it reached room temperature. Then, a saturated solution of $\mathrm{NH}_{4} \mathrm{Cl}(6 \mathrm{~mL})$ was added. The reaction mixture was dissolved in EtOAc and washed with $\mathrm{H}_{2} \mathrm{O}$, brine and $10 \%$ aqueous $\mathrm{Na}_{2} \mathrm{~S}_{2} \mathrm{O}_{3}$ solution. Then, the mixture was dried with $\mathrm{Na}_{2} \mathrm{SO}_{4}$ and, after being filtered, the solvent was evaporated. The resulting mixture $(4.41 \mathrm{~g})$ was chromatographed and the desired compounds eluted with hexane/EtOAc 95:5. Yield: $141 \mathrm{mg}$ (23\% yield) of 3 and $217 \mathrm{mg}$ (45\% yield) of 4 .

Pentan-3-yl (1R,2R,5S)-2-(benzyl((R)-1-phenylethyl)amino)-5-((1S,2R)-1-hydroxy-3-oxo-3-(pentan3-yloxy) -1-phenylpropan-2-yl)cyclopentane-1-carboxylate $(3):[\alpha]_{\mathrm{D}}^{26}=+5.11\left(\mathrm{CHCl}_{3}, \mathrm{c}=0.89\right)$. IR $\left(\mathrm{cm}^{-1}\right): 3495,2969,1723,1460,748,698$. H.R.M.S.: calcd for $\mathrm{C}_{40} \mathrm{H}_{53} \mathrm{NO}_{5}$ : 627.3924; found: 627.3895 . ${ }^{1} \mathrm{H}-\mathrm{NMR} \delta(\mathrm{ppm})\left(400 \mathrm{MHz}, \mathrm{CDCl}_{3}\right): 0.40\left(3 \mathrm{H}, \mathrm{t}, J=7.3 \mathrm{~Hz}, \mathrm{CH}\left(\mathrm{CH}_{2} \mathrm{CH}_{3}\right)_{2}\right), 0.78(3 \mathrm{H}, \mathrm{t}, J=7.3 \mathrm{~Hz}$, $\left.\mathrm{CH}\left(\mathrm{CH}_{2} \mathrm{CH}_{3}\right)_{2}\right), 0.80\left(3 \mathrm{H}, \mathrm{t}, J=7.3 \mathrm{~Hz}, \mathrm{CH}\left(\mathrm{CH}_{2} \mathrm{CH}_{3}\right)_{2}\right), 0.95\left(3 \mathrm{H}, \mathrm{t}, J=7.3 \mathrm{~Hz}, \mathrm{CH}\left(\mathrm{CH}_{2} \mathrm{CH}_{3}\right)_{2}\right), 1.32$ $\left(3 \mathrm{H}, \mathrm{d}, J=7.0 \mathrm{~Hz}, \mathrm{H}-2^{\prime}\right), 1.55$ (2H, m, H-4), 1.71 (2H, m, H-3), 2.62 (1H, m, H-1), 2.64 (1H, m, H-5), $2.71(1 \mathrm{H}, \mathrm{m}, \mathrm{H}-7), 3.59(1 \mathrm{H}, \mathrm{q}, J=7.6 \mathrm{~Hz}, \mathrm{H}-2), 3.68\left(1 \mathrm{H}, \mathrm{d}, J=14.6 \mathrm{~Hz}, \mathrm{H}-1^{\prime \prime} \mathrm{A}\right), 3.72(1 \mathrm{H}, \mathrm{d}, J=14.6$ $\left.\mathrm{Hz}, \mathrm{H}-1^{\prime \prime} \mathrm{B}\right), 3.86\left(1 \mathrm{H}, \mathrm{q}, J=7.0 \mathrm{~Hz}, \mathrm{H}-1^{\prime}\right), 3.92(1 \mathrm{H}, \mathrm{d}, J=9.1 \mathrm{~Hz}, \mathrm{OH}), 4.57(2 \mathrm{H}$, quintet, $J=6.1 \mathrm{~Hz}$, $\left.\mathrm{CH}\left(\mathrm{CH}_{2} \mathrm{CH}_{3}\right)_{2 \times} 2\right), 4.87\left(1 \mathrm{H}, \mathrm{dd}, J=9.1\right.$ and $\left.3.7 \mathrm{~Hz}, \mathrm{H}-1^{\prime \prime \prime}\right), 7.23-7.29(15 \mathrm{H}, \mathrm{Ar}) .{ }^{13} \mathrm{C}-\mathrm{NMR} \delta(\mathrm{ppm})$ $\left(\mathrm{CDCl}_{3}\right): 8.6\left(\mathrm{CH}_{3}, \mathrm{CH}\left(\mathrm{CH}_{2} \mathrm{CH}_{3}\right)_{2}\right), 9.2\left(\mathrm{CH}_{3}, \mathrm{CH}\left(\mathrm{CH}_{2} \mathrm{CH}_{3}\right)_{2} \times 2\right), 9.5\left(\mathrm{CH}_{3}, \mathrm{CH}\left(\mathrm{CH}_{2} \mathrm{CH}_{3}\right)_{2}\right), 15.2,\left(\mathrm{CH}_{3}\right.$, C-2' $), 25.3\left(\mathrm{CH}_{2}, \mathrm{CH}\left(\mathrm{CH}_{2} \mathrm{CH}_{3}\right)_{2} \times 3\right), 25.6\left(\mathrm{CH}_{2}, \mathrm{CH}\left(\mathrm{CH}_{2} \mathrm{CH}_{3}\right)_{2}\right), 27.3\left(\mathrm{CH}_{2}, \mathrm{C}-4\right), 28.8\left(\mathrm{CH}_{2}, \mathrm{C}-3\right), 41.5$ $(\mathrm{CH}, \mathrm{C}-5), 50.0\left(\mathrm{CH}_{2}, \mathrm{C}-1^{\prime \prime}\right), 52.4(\mathrm{CH}, \mathrm{C}-1), 55.1(\mathrm{CH}, \mathrm{C}-7), 57.3\left(\mathrm{CH}, \mathrm{C}-1^{\prime}\right), 64.8(\mathrm{CH}, \mathrm{C}-2), 72.2(\mathrm{CH}$, $\left.\mathrm{C}-1^{\prime \prime \prime}\right), 76.5\left(\mathrm{CH}, \mathrm{CH}\left(\mathrm{CH}_{2} \mathrm{CH}_{3}\right)_{2}\right), 77.6\left(\mathrm{CH}, \mathrm{CH}\left(\mathrm{CH}_{2} \mathrm{CH}_{3}\right)_{2}\right), 125.6$ - $128.6(\mathrm{CHx} 10, \mathrm{Ar}), 141.4\left(\mathrm{C}, \mathrm{C}_{\mathrm{ipso}}\right)$, $142.4\left(\mathrm{C}, \mathrm{C}_{\mathrm{ipso}}\right), 143.9$ (C, $\left.\mathrm{C}_{\mathrm{ipso}}\right), 174.5$ (COOR, C-6), 182,4 (COOR, C-8). 
Pentan-3-yl (1R,2R,5S)-2-(benzyl((R)-1-phenylethyl)amino)-5-((1R,2R)-1-hydroxy-3-oxo-3-(pentan3-yl- oxy)-1-phenylpropan-2-yl)cyclopentane-1-carboxylate (4): $[\alpha]_{\mathrm{D}}{ }^{26}=+0.90\left(\mathrm{CHCl}_{3}, \mathrm{c}=1.29\right)$. H.R.M.S.: calcd for $\mathrm{C}_{40} \mathrm{H}_{53} \mathrm{NO}_{5}$ : 627.3924; found: 627.3864. IR $\left(\mathrm{cm}^{-1}\right)$ : 3495, 2969, 1723, 1495, 1169, 912, 748, 698. ${ }^{1} \mathrm{H}-\mathrm{NMR} \delta(\mathrm{ppm})\left(400 \mathrm{MHz}, \mathrm{CDCl}_{3}\right): 0.32\left(3 \mathrm{H}, \mathrm{t}, J=7.5 \mathrm{~Hz}, \mathrm{CH}\left(\mathrm{CH}_{2} \mathrm{CH}_{3}\right)_{2}\right), 0.73$ $\left(3 \mathrm{H}, \mathrm{t}, J=7.5 \mathrm{~Hz}, \mathrm{CH}\left(\mathrm{CH}_{2} \mathrm{CH}_{3}\right)_{2}\right), 0.98\left(3 \mathrm{H}, \mathrm{t}, J=7.5 \mathrm{~Hz}, \mathrm{CH}\left(\mathrm{CH}_{2} \mathrm{CH}_{3}\right)_{2}\right), 1.00(3 \mathrm{H}, \mathrm{t}, J=7.5 \mathrm{~Hz}$, $\left.\mathrm{CH}\left(\mathrm{CH}_{2} \mathrm{CH}_{3}\right)_{2}\right), 1.08\left(2 \mathrm{H}, \mathrm{m}, \mathrm{CH}\left(\mathrm{CH}_{2} \mathrm{CH}_{3}\right)_{2}\right), 1.28\left(3 \mathrm{H}, \mathrm{d}, \mathrm{J}=6.8 \mathrm{~Hz}, \mathrm{H}-2^{\prime}\right), 1.35\left(2 \mathrm{H}, \mathrm{m}, \mathrm{CH}\left(\mathrm{CH}_{2} \mathrm{CH}_{3}\right)_{2}\right)$, $1.55(1 \mathrm{H}, \mathrm{m}, \mathrm{H}-4 \mathrm{~A}), 1.64\left(2 \mathrm{H}, \mathrm{m}, \mathrm{CH}\left(\mathrm{CH}_{2} \mathrm{CH}_{3}\right)_{2}\right), 1.65\left(2 \mathrm{H}, \mathrm{m}, \mathrm{CH}\left(\mathrm{CH}_{2} \mathrm{CH}_{3}\right)_{2}\right), 1.75(2 \mathrm{H}, \mathrm{m}, \mathrm{H}-3), 1.85$ $(1 \mathrm{H}, \mathrm{m}, \mathrm{H}-4 \mathrm{~B}), 2.74(2 \mathrm{H}, \mathrm{m}, \mathrm{H}-5), 2.87(1 \mathrm{H}, \mathrm{dd}, J=10.0$ and $3.8 \mathrm{~Hz}, \mathrm{H}-7), 3.04(1 \mathrm{H}, \mathrm{d}, J=10.0 \mathrm{~Hz}, \mathrm{OH})$, $3.23(1 \mathrm{H}, \mathrm{dd}, J=10.1$ and $9.9 \mathrm{~Hz}, \mathrm{H}-1), 3.56(1 \mathrm{H}, \mathrm{ddd}, J=9.9,8.2$ and $8.2 \mathrm{~Hz}, \mathrm{H}-2), 3.67(1 \mathrm{H}, \mathrm{d}, J=14.4$ $\left.\mathrm{Hz}, \mathrm{H}-1^{\prime \prime} \mathrm{A}\right), 3.85\left(1 \mathrm{H}, \mathrm{d}, J=14.4 \mathrm{~Hz}, \mathrm{H}-1^{\prime \prime} \mathrm{B}\right), 3.90\left(1 \mathrm{H}, \mathrm{q}, J=6.8 \mathrm{~Hz}, \mathrm{H}-1^{\prime}\right), 4.44(1 \mathrm{H}$, quintet, $J=6.0 \mathrm{~Hz}$, $\left.\mathrm{CH}\left(\mathrm{CH}_{2} \mathrm{CH}_{3}\right)_{2},\right), 4.64\left(1 \mathrm{H}, \mathrm{dd}, J=10.0\right.$ and $\left.3.5 \mathrm{~Hz}, \mathrm{H}-1^{\prime \prime \prime}\right), 4.67\left(1 \mathrm{H}\right.$, quintet, $\left.J=6.0 \mathrm{~Hz}, \mathrm{CH}\left(\mathrm{CH}_{2} \mathrm{CH}_{3}\right)_{2}\right)$, 7.1-7.6 (15H, Ar-H). ${ }^{13} \mathrm{C}-\mathrm{NMR} \delta(\mathrm{ppm})\left(\mathrm{CDCl}_{3}\right): 8.6\left(\mathrm{CH}_{3}, \mathrm{CH}\left(\mathrm{CH}_{2} \mathrm{CH}_{3}\right)_{2}\right), 9.0\left(\mathrm{CH}_{3}, \mathrm{CH}\left(\mathrm{CH}_{2} \mathrm{CH}_{3}\right)_{2}\right)$, $9.2\left(\mathrm{CH}_{3}, \mathrm{CH}\left(\mathrm{CH}_{2} \mathrm{CH}_{3}\right)_{2}\right) 9.4\left(\mathrm{CH}_{3}, \mathrm{CH}\left(\mathrm{CH}_{2} \mathrm{CH}_{3}\right)_{2}\right), 15,1\left(\mathrm{CH}_{3}, \mathrm{C}-2^{\prime}\right), 24.5\left(\mathrm{CH}_{2}, \mathrm{CH}\left(\mathrm{CH}_{2} \mathrm{CH}_{3}\right)_{2}\right), 25.0$ $\left(\mathrm{CH}_{2}, \mathrm{CH}\left(\mathrm{CH}_{2} \mathrm{CH}_{3}\right)_{2}\right), 25.2\left(\mathrm{CH}_{2}, \mathrm{CH}\left(\mathrm{CH}_{2} \mathrm{CH}_{3}\right)_{2}\right), 25.4\left(\mathrm{CH}_{2}, \mathrm{CH}\left(\mathrm{CH}_{2} \mathrm{CH}_{3}\right)_{2}\right), 26.6\left(\mathrm{CH}_{2}, \mathrm{C}-3\right), 28.8$ $\left(\mathrm{CH}_{2}, \mathrm{C}-4\right), 40.0(\mathrm{CH}, \mathrm{C}-5), 49.9\left(\mathrm{CH}_{2}, \mathrm{C}-1^{\prime \prime}\right), 50.0(\mathrm{CH}, \mathrm{C}-1), 56.5\left(\mathrm{CH}_{2}, \mathrm{C}-7\right), 57.2\left(\mathrm{CH}, \mathrm{C}-1^{\prime}\right), 65.1(\mathrm{CH}$, $\mathrm{C}-2), 73.3\left(\mathrm{CH}, \mathrm{C}-1^{\prime \prime \prime}\right), 77.1\left(\mathrm{CH}, \mathrm{CH}\left(\mathrm{CH}_{2} \mathrm{CH}_{3}\right)_{2}\right), 77.4\left(\mathrm{CH}, \mathrm{CH}\left(\mathrm{CH}_{2} \mathrm{CH}_{3}\right)_{2}\right), 126.5$ - $128.8(\mathrm{CHx} 15, \mathrm{Ar})$, 141.3 (C, C ipso $), 141.6$ (C, C ipso), 144.1 (C, C ipso), 172.0 (COOR, C-8), 177.4 (COOR, C-6).

To a solution of $3(121 \mathrm{mg}, 0.193 \mathrm{mmol})$ in $\mathrm{CH}_{2} \mathrm{Cl}_{2}(3 \mathrm{~mL})$ under an Ar atmosphere and at $-78{ }^{\circ} \mathrm{C}$, $1.5 \mathrm{M}$ DIBALH $(0.4 \mathrm{~mL}, 0.58 \mathrm{mmol})$ was added. After $2 \mathrm{~h}$ of reaction, $\mathrm{H}_{2} \mathrm{O}(1.5 \mathrm{~mL})$ was added and the resulting mixture was washed in an Erlenmeyer flask with $\mathrm{NaHCO}_{3}$ and $\mathrm{Na}_{2} \mathrm{SO}_{4}$ (3 g of each) for $5 \mathrm{~h}$. After being filtered, the solvent was evaporated and $101 \mathrm{mg}$ of crude product were obtained. The desired compounds were subjected to chromatography eluting with hexane/EtOAc 9:1 to afford $59 \mathrm{mg}$ of 5 (yield $57 \%$ ), $25 \mathrm{mg}$ of $\mathbf{6}$ (yield $24 \%$ ) and $8 \mathrm{mg}$ of 7 (yield $9 \%$ ).

Pentan-3-yl (2R,3R)-2-((1S,2R,3R)-3-(benzyl((R)-1-phenylethyl)amino)-2-(hydroxymethyl)cyclopentyl)-3hydroxy-3-phenylpropanoate (5): IR $\left(\mathrm{cm}^{-1}\right)$ : 3368, 2969, 1717, 1456, 1169, 1028, 750, 698. H.R.M.S.: calcd for $\mathrm{C}_{35} \mathrm{H}_{45} \mathrm{NO}_{4}$ : 543.3349; found: 543.3398. ${ }^{1} \mathrm{H}-\mathrm{NMR} \delta(\mathrm{ppm})\left(400 \mathrm{MHz}, \mathrm{CDCl}_{3}\right): 0.37(3 \mathrm{H}, \mathrm{t}, J=7.5$ $\left.\mathrm{Hz}, \mathrm{CH}\left(\mathrm{CH}_{2} \mathrm{CH}_{3}\right)_{2}\right), 0.78\left(3 \mathrm{H}, \mathrm{t}, J=7.5 \mathrm{~Hz}, \mathrm{CH}\left(\mathrm{CH}_{2} \mathrm{CH}_{3}\right)_{2}\right), 1.13\left(3 \mathrm{H}, \mathrm{m}, \mathrm{CH}\left(\mathrm{CH}_{2} \mathrm{CH}_{3}\right)_{2}\right), 1.38(3 \mathrm{H}, \mathrm{d}, J$ $\left.=6.8 \mathrm{~Hz}, \mathrm{H}-2^{\prime}\right), 1.35\left(3 \mathrm{H}, \mathrm{m}, \mathrm{CH}\left(\mathrm{CH}_{2} \mathrm{CH}_{3}\right)_{2}\right), 2.24(1 \mathrm{H}, \mathrm{m}, \mathrm{H}-1), 2.24(1 \mathrm{H}, \mathrm{m}, \mathrm{H}-5), 2.84(1 \mathrm{H}, \mathrm{m}, \mathrm{H}-7)$, $2.84(1 \mathrm{H}, \mathrm{m}, \mathrm{H}-2), 3.44(1 \mathrm{H}, \mathrm{m}, \mathrm{H}-6 \mathrm{~A}), 3.55(1 \mathrm{H}, \mathrm{m}, \mathrm{H}-6 \mathrm{~B}), 3.58$ (1H, d, J = 14.4 Hz, H-1"'A), 3.91 (1H, d, $\left.J=14.4 \mathrm{~Hz}, \mathrm{H}-1^{\prime \prime} \mathrm{B}\right), 3.94\left(1 \mathrm{H}, \mathrm{q}, J=6.8 \mathrm{~Hz}, \mathrm{H}-1^{\prime}\right), 4.70\left(1 \mathrm{H}\right.$, quintet, $\left.J=8 \mathrm{~Hz}, \mathrm{CH}\left(\mathrm{CH}_{2} \mathrm{CH}_{3}\right)_{2}\right), 4.96(1 \mathrm{H}$, $\left.\mathrm{d}, J=11.6 \mathrm{~Hz}, \mathrm{H}-1^{\prime \prime \prime}\right), 7.22-7.40(15 \mathrm{H}, \mathrm{Ar}-\mathrm{H}) .{ }^{13} \mathrm{C}-\mathrm{NMR} \delta(\mathrm{ppm})\left(\mathrm{CDCl}_{3}\right): 8.6\left(\mathrm{CH}_{3}, \mathrm{CH}\left(\mathrm{CH}_{2} \mathrm{CH}_{3}\right)_{2}\right), 9.7$ $\left(\mathrm{CH}_{3}, \mathrm{CH}\left(\mathrm{CH}_{2} \mathrm{CH}_{3}\right)_{2}\right), 12.4\left(\mathrm{CH}_{3}, \mathrm{C}-2^{\prime}\right), 25.3\left(\mathrm{CH}_{2}, \mathrm{CH}\left(\mathrm{CH}_{2} \mathrm{CH}_{3}\right)_{2}\right), 25.4\left(\mathrm{CH}_{2}, \mathrm{CH}\left(\mathrm{CH}_{2} \mathrm{CH}_{3}\right)_{2}\right), 25.7$ $\left(\mathrm{CH}_{2}, \mathrm{C}-3\right), 28.5\left(\mathrm{CH}_{2}, \mathrm{C}-4\right), 36.1(\mathrm{CH}, \mathrm{C}-5), 45.1(\mathrm{CH}, \mathrm{C}-1), 50.1\left(\mathrm{CH}_{2}, \mathrm{C}-1^{\prime \prime}\right), 55.4\left(\mathrm{CH}, \mathrm{C}-1^{\prime}\right), 57.2(\mathrm{CH}$, C-7), $60.7(\mathrm{CH}, \mathrm{C}-2), 64.7(\mathrm{CH}, \mathrm{C}-6), 73.3\left(\mathrm{CH}, \mathrm{C}-1^{\prime \prime \prime}\right), 76.8\left(\mathrm{CH}, \mathrm{CH}\left(\mathrm{CH}_{2} \mathrm{CH}_{3}\right)_{2}\right), 126.8$ - 128.9 (CHx15, Ar), 140.8 (C, $\left.\mathrm{C}_{i p s o}\right), 142.1$ (C, $\left.\mathrm{C}_{i p s o}\right), 144.0\left(\mathrm{C}, \mathrm{C}_{i p s o}\right), 173.0$ (COOR, C-8).

Pentan-3-yl (1R,2R,5R)-2-(benzyl((R)-1-phenylethyl)amino)-5-((1R,2S)-1,3-dihydroxy-1-phenylpropan-2-yl)-cyclopentane-1-carboxylate (6): $[\alpha]_{\mathrm{D}}{ }^{26}=-4.5\left(\mathrm{CHCl}_{3}, \mathrm{c}=0.79\right)$. IR $\left(\mathrm{cm}^{-1}\right): 3422,2969$, 1717, 1486, 1028, 750, 667. H.R.M.S.: calcd for $\mathrm{C}_{35} \mathrm{H}_{45} \mathrm{NO}_{4}$ : 559.3662; found: 543.3293. ${ }^{1} \mathrm{H}-\mathrm{NMR} \delta$ (ppm) $\left(400 \mathrm{MHz}, \mathrm{CDCl}_{3}\right): 0.83\left(3 \mathrm{H}, \mathrm{t}, J=7.6 \mathrm{~Hz}, \mathrm{CH}\left(\mathrm{CH}_{2} \mathrm{CH}_{3}\right)_{2}\right), 0.89\left(3 \mathrm{H}, \mathrm{t}, J=7.6 \mathrm{~Hz}, \mathrm{CH}\left(\mathrm{CH}_{2} \mathrm{CH}_{3}\right)_{2}\right), 1.26$ $(1 \mathrm{H}, \mathrm{m}, \mathrm{H}-4 \mathrm{~A}), 1.31\left(4 \mathrm{H}, \mathrm{m}, \mathrm{CH}\left(\mathrm{CH}_{2} \mathrm{CH}_{3}\right)_{2} \times 2\right), 1.34\left(3 \mathrm{H}, \mathrm{d}, J=6.8 \mathrm{~Hz}, \mathrm{H}-2^{\prime}\right), 1.65(1 \mathrm{H}, \mathrm{m}, \mathrm{H}-3 \mathrm{~A}), 1.75$ (1H, m, H-3B), 1.80 (1H, m, H-4B), $1.99(1 \mathrm{H}, \mathrm{m}, \mathrm{H}-7), 2.27(1 \mathrm{H}, \mathrm{m}, \mathrm{H}-5), 2.73(1 \mathrm{H}, \mathrm{t}, J=9.1 \mathrm{~Hz}, \mathrm{H}-1), 3.51$ $(1 \mathrm{H}, \mathrm{d}, J=4.5 \mathrm{~Hz}, \mathrm{H}-8 \mathrm{~A}), 3.55(1 \mathrm{H}, \mathrm{m}, \mathrm{H}-2), 3.69\left(1 \mathrm{H}, \mathrm{d}, J=14.6, \mathrm{H}-1^{\prime \prime} \mathrm{A}\right), 3.77\left(1 \mathrm{H}, \mathrm{d}, J=14.6 \mathrm{~Hz}, \mathrm{H}-1^{\prime \prime} \mathrm{B}\right)$, $3.82(1 \mathrm{H}, \mathrm{m}, \mathrm{H}-8 \mathrm{~B}), 3.90\left(1 \mathrm{H}, \mathrm{q}, J=6.8 \mathrm{~Hz}, \mathrm{H}-\mathrm{1}^{\prime}\right), 4.58\left(1 \mathrm{H}\right.$, quintet, $\left.J=5.9 \mathrm{~Hz}, \mathrm{CH}\left(\mathrm{CH}_{2} \mathrm{CH}_{3}\right)_{2}\right), 4.86(1 \mathrm{H}$, $\left.\mathrm{d}, J=6.5 \mathrm{~Hz}, \mathrm{H}-1^{\prime \prime} \mathrm{B}\right), 7.2-7.6(15 \mathrm{H}, \mathrm{Ar}-\mathrm{H}) .{ }^{13} \mathrm{C}-\mathrm{NMR} \delta(\mathrm{ppm})\left(\mathrm{CDCl}_{3}\right): 9.0\left(\mathrm{CH}_{3}, \mathrm{CH}\left(\mathrm{CH}_{2} \mathrm{CH}_{3}\right)_{2}\right), 9.2$ $\left(\mathrm{CH}_{3}, \mathrm{CH}\left(\mathrm{CH}_{2} \mathrm{CH}_{3}\right)_{2}\right), 15.1\left(\mathrm{CH}_{3}, \mathrm{C}-2^{\prime}\right), 24.8\left(\mathrm{CH}_{2}, \mathrm{CH}\left(\mathrm{CH}_{2} \mathrm{CH}_{3}\right)_{2}\right), 25.1\left(\mathrm{CH}_{2}, \mathrm{CH}\left(\mathrm{CH}_{2} \mathrm{CH}_{3}\right)_{2}\right), 27.1$ $\left(\mathrm{CH}_{2}, \mathrm{C}-3\right), 30.3\left(\mathrm{CH}_{2}, \mathrm{C}-4\right), 39.8(\mathrm{CH}, \mathrm{C}-5), 50.0\left(\mathrm{CH}_{2}, \mathrm{C}-1^{\prime \prime}\right), 50.6(\mathrm{CH}, \mathrm{C}-7), 53.0(\mathrm{CH}, \mathrm{C}-1), 58.0(\mathrm{CH}$, C-1'), $63.4\left(\mathrm{CH}_{2}, \mathrm{C}-8\right), 65.1(\mathrm{CH}, \mathrm{C}-2), 75.6\left(\mathrm{CH}, \mathrm{C}-1^{\prime \prime \prime}\right), 77.0\left(\mathrm{CH}, \mathrm{CH}\left(\mathrm{CH}_{2} \mathrm{CH}_{3}\right)_{2}\right), 125.3$ - $128.7(\mathrm{CHx} 15$, Ar), 141.5 (C, $\left.C_{\text {ipso }}\right), 142.7$ (C, C ipso $), 143.9$ (C, C ipso $\left._{\text {) }}\right), 176.6$ (COOR, C-6).

$(1 R, 2 S)-2-((1 S, 2 R, 3 R)-3-($ benzyl((R)-1-phenylethyl)amino)-2-(hydroxymethyl)cyclopentyl)-1-phenylpropane-1,3-diol (7): IR $\left(\mathrm{cm}^{-1}\right)$ : 3339, 2940, 1495, 1028, 737, 700. H.R.M.S.: calcd for $\mathrm{C}_{30} \mathrm{H}_{37} \mathrm{NO}_{3}$ : 
459.2773; found: $459.2735{ }^{1} \mathrm{H}-\mathrm{NMR} \delta(\mathrm{ppm})\left(200 \mathrm{MHz}, \mathrm{CDCl}_{3}\right): 1.26(1 \mathrm{H}, \mathrm{m}, \mathrm{H}-7), 1.36(3 \mathrm{H}, \mathrm{d}, J=6.8$ Hz, H-2'), 1.46-1.87 (2H, m, H-3; 1H, m, H-4A, 1H, m, H-5) 1.92 (1H, m, H-4B), 1.95 (1H, m, H-1), 2.83 $(1 \mathrm{H}, \mathrm{q}, J=8 \mathrm{~Hz}, \mathrm{H}-2), 3.28(1 \mathrm{H}, \mathrm{m}, \mathrm{H}-6 \mathrm{~A}), 3.36(1 \mathrm{H}, \mathrm{m}, \mathrm{H}-6 \mathrm{~B}), 3.60(2 \mathrm{H}, \mathrm{m}, \mathrm{H}-8), 3.62(1 \mathrm{H}, \mathrm{d}, J=7.0 \mathrm{~Hz}$, $\left.\mathrm{H}-1^{\prime \prime} \mathrm{B}\right), 3.90\left(1 \mathrm{H}, \mathrm{q}, J=6.8 \mathrm{~Hz}, \mathrm{H}-1^{\prime}\right), 3.94\left(1 \mathrm{H}, \mathrm{d}, J=7.0 \mathrm{~Hz}, \mathrm{H}-1^{\prime \prime} \mathrm{A}\right), 4.94\left(1 \mathrm{H}, \mathrm{d}, J=5.8 \mathrm{~Hz}, \mathrm{H}-1^{\prime \prime \prime}\right)$, 7.21-7.38 (15 H, Ar-H). ${ }^{13} \mathrm{C}-\mathrm{NMR} \delta(\mathrm{ppm})\left(\mathrm{CDCl}_{3}\right): 12.9\left(\mathrm{CH}_{3}, \mathrm{C}-2^{\prime}\right), 25.9\left(\mathrm{CH}_{2}, \mathrm{C}-3\right), 29.0\left(\mathrm{CH}_{2}, \mathrm{C}-4\right)$, $36.1(\mathrm{CH}, \mathrm{C}-5), 46.8(\mathrm{CH}, \mathrm{C}-1), 50.3\left(\mathrm{CH}_{2}, \mathrm{C}-1^{\prime \prime}\right), 56.5\left(\mathrm{CH}, \mathrm{C}-1^{\prime}\right), 50.7(\mathrm{CH}, \mathrm{C}-7), 62.4(\mathrm{CH}, \mathrm{C}-2), 62.8$ $\left(\mathrm{CH}_{2}, \mathrm{C}-8\right), 66.0(\mathrm{CH} 2, \mathrm{C}-6), 75.3\left(\mathrm{CH}, \mathrm{C}-1^{\prime \prime \prime}\right), 126.5-129.3$ (CHx15, Ar), $140.7\left(\mathrm{C}, \mathrm{C}_{i p s o}\right), 143.4\left(\mathrm{C}, \mathrm{C}_{i p s o}\right)$, $143.6\left(\mathrm{C}, \mathrm{C}_{i p s o}\right)$.

Also, to a solution of $3(147 \mathrm{mg}, 0.235 \mathrm{mmol})$ in dry ether $(5 \mathrm{~mL})$ at $0{ }^{\circ} \mathrm{C}, \mathrm{LiAlH}_{4}(24 \mathrm{mg})$ was added and, after $45 \mathrm{~min}$ of stirring, dry ether saturated with water $(1 \mathrm{~mL})$ was added. The reaction mixture was filtered through Celite-silica and washed with ether and $\mathrm{CHCl}_{3}$. The solvent was evaporated and $101 \mathrm{mg}$ of crude product were obtained and chromatographed. $20 \mathrm{mg}$ of 5 (yield 16\%), $39 \mathrm{mg}$ of 6 (yield $31 \%$ ) and $16 \mathrm{mg}$ of 7 (yield $15 \%$ ).

To a solution of $3+4$ (2:3 ratio) $(86 \mathrm{mg}, 0.137 \mathrm{mmol})$ in DCM ( $3 \mathrm{~mL}), 1.5 \mathrm{M}$ DIBALH $(1.12 \mathrm{~mL}$, $1.680 \mathrm{mmol}$ ) was added at $-78{ }^{\circ} \mathrm{C}$ under an Ar atmosphere. After $1 \mathrm{~h}$, the reaction flask was allowed to reach room temperature and $\mathrm{H}_{2} \mathrm{O}(1.5 \mathrm{~mL})$ was added. Then, the reaction mixture was placed in an Erlenmeyer flask with ether, $\mathrm{NaHCO}_{3}(3 \mathrm{~g})$ and $\mathrm{Na}_{2} \mathrm{SO}_{4}(3 \mathrm{~g})$ and the resulting mixture left stirring for $5 \mathrm{~h}$. Then, it was chromatographed and the desired products were eluted with hexane/EtOAc 8:2 to give $26 \mathrm{mg}$ of $\mathbf{7}$ (yield $38 \%$ ) and $18 \mathrm{mg}$ of $\mathbf{8}$ (yield $27 \%$ ).

(1S,2S)-2-((1S,2R,3R)-3-(benzyl((R)-1-phenylethyl)amino)-2-(hydroxymethyl)cyclopentyl)-1-phenylpropane-1,3-diol (8): I.R. $\left(\mathrm{cm}^{-1}\right)$ : 3341, 2930, 1495, 1059, 750, 700. ${ }^{1} \mathrm{H}-\mathrm{NMR} \delta$ (ppm) $\left(400 \mathrm{MHz}, \mathrm{CDCl}_{3}\right)$ : $1.25(1 \mathrm{H}, \mathrm{H}-7), 1.39\left(3 \mathrm{H}, \mathrm{d}, J=6.9 \mathrm{~Hz}, \mathrm{H}-2^{\prime}\right), 1.44(1 \mathrm{H}, \mathrm{m}, \mathrm{H}-5), 1.67(1 \mathrm{H}, \mathrm{m}, \mathrm{H}-4 \mathrm{~A}), 1.74(2 \mathrm{H}, \mathrm{m}, \mathrm{H}-3)$, $1.77(1 \mathrm{H}, \mathrm{m}, \mathrm{H}-5), 1.86(1 \mathrm{H}, \mathrm{m}, \mathrm{H}-4 \mathrm{~B}), 2.11(1 \mathrm{H}, \mathrm{m}, \mathrm{H}-1), 2.83(1 \mathrm{H}, \mathrm{q}, J=8 \mathrm{~Hz}, \mathrm{H}-2), 3.33(2 \mathrm{H}, \mathrm{m}, \mathrm{H}-6)$, $3.63\left(1 \mathrm{H}, \mathrm{d}, J=13.8 \mathrm{~Hz}, \mathrm{H}-1^{\prime \prime} \mathrm{A}\right), 3.64(2 \mathrm{H}, \mathrm{m}, \mathrm{H}-8), 3.78\left(1 \mathrm{H}, \mathrm{d}, J=13.8 \mathrm{~Hz}, \mathrm{H}-1^{\prime \prime} \mathrm{B}\right), 3.92(1 \mathrm{H}, \mathrm{q}, J=6.9$ $\left.\mathrm{Hz}, \mathrm{H}-1^{\prime}\right), 5.00\left(1 \mathrm{H}, \mathrm{d}, J=3.1 \mathrm{~Hz}, \mathrm{H}-1^{\prime \prime \prime}\right), 7.21-7.40(15 \mathrm{H}, \mathrm{Ar}-\mathrm{H}) .{ }^{13} \mathrm{C}-\mathrm{NMR} \delta(\mathrm{ppm})\left(\mathrm{CDCl}_{3}\right): 13.2\left(\mathrm{CH}_{3}\right.$, C-2'), $25.9\left(\mathrm{CH}_{2}, \mathrm{C}-3\right), 28.8\left(\mathrm{CH}_{2}, \mathrm{C}-4\right), 36.5(\mathrm{CH}, \mathrm{C}-5), 47.3(\mathrm{CH}, \mathrm{C}-1), 50.7\left(\mathrm{CH}_{2}, \mathrm{C}-1^{\prime \prime}\right), 56.5\left(\mathrm{CH}, \mathrm{C}-1^{\prime}\right)$, $50.1(\mathrm{CH}, \mathrm{C}-7), 62.3(\mathrm{CH}, \mathrm{C}-2), 60.9\left(\mathrm{CH}_{2}, \mathrm{C}-8\right), 66.0\left(\mathrm{CH}_{2}, \mathrm{C}-6\right), 76.5\left(\mathrm{CH}, \mathrm{C}-1^{\prime \prime \prime}\right), 126.6-129.1$ (CHx15, Ar), 140.8 (C, $\left.\mathrm{C}_{i p s o}\right), 143.9$ (C, $\left.\mathrm{C}_{i p s o}\right), 144.8\left(\mathrm{C}, \mathrm{C}_{i p s o}\right)$.

To a solution of $7(22 \mathrm{mg}, 0.048 \mathrm{mmol})$ in acetone $(5 \mathrm{~mL})$, a catalytic amount of camphorsulfonic acid (CSA) and 2,2-DMP (5 mL) were added. The reaction was heated up to reflux for $7 \mathrm{~h}$. The reaction mixture was solved in ether and washed with $\mathrm{NaHCO}_{3}$ saturated solution, brine and $\mathrm{H}_{2} \mathrm{O}$. The resulting solution was dried with $\mathrm{Na}_{2} \mathrm{SO}_{4}$ and, after being filtered, $42 \mathrm{mg}$ of crude product were obtained and chromatographed. The desired compound was eluted with hexane/EtOAc 95:5, and 12 mg of 9 were obtained (yield $65 \%$ ).

$((1 R, 2 R, 5 S)-2-($ benzyl((R)-1-phenylethyl)amino)-5-((4R,5S)-2,2-dimethyl-4-phenyl-1,3-dioxan-5-yl)cyclopentyl)methanol (9): IR $\left(\mathrm{cm}^{-1}\right)$ : 3332, 2938, 1451, 1200, 739, 700. H.R.M.S.: calcd for $\mathrm{C}_{33} \mathrm{H}_{41} \mathrm{NO}_{3}$ : 499.3086; found: 499.3044. ${ }^{1} \mathrm{H}-\mathrm{NMR} \delta(\mathrm{ppm})\left(400 \mathrm{MHz}, \mathrm{CDCl}_{3}\right): 0.22(1 \mathrm{H}, \mathrm{m}, \mathrm{H}-4 \mathrm{~A}), 0.88(1 \mathrm{H}, \mathrm{m}, \mathrm{H}-5)$, $1.17(1 \mathrm{H}, \mathrm{m}, \mathrm{H}-4 \mathrm{~B}), 1.27\left(3 \mathrm{H}, \mathrm{d}, J=6.8 \mathrm{~Hz}, \mathrm{H}-2^{\prime}\right), 1.28-1.40(1 \mathrm{H}, \mathrm{m}, \mathrm{H}-3 \mathrm{~A}), 1.47\left(3 \mathrm{H}, \mathrm{s},\left(\mathrm{CH}_{3}\right)_{2}\right), 1.49(3 \mathrm{H}$, $\left.\mathrm{s},\left(\mathrm{CH}_{3}\right)_{2}\right), 1.57(1 \mathrm{H}, \mathrm{m}, \mathrm{H}-7), 1.68(1 \mathrm{H}, \mathrm{m}, \mathrm{H}-3 \mathrm{~B}), 1.68(1 \mathrm{H}, \mathrm{m}, \mathrm{H}-1), 2.71(1 \mathrm{H}, \mathrm{q}, J=8, \mathrm{H}-2), 3.08(1 \mathrm{H}, \mathrm{dd}$, $J=10.5$ and $6.5 \mathrm{~Hz}, \mathrm{H}-6 \mathrm{~A}), 3.47\left(1 \mathrm{H}, \mathrm{d}, J=13.5 \mathrm{~Hz}, \mathrm{H}-1^{\prime \prime} \mathrm{A}\right), 3.62(1 \mathrm{H}, \mathrm{dd}, J=10.5$ and $2.4 \mathrm{~Hz}, \mathrm{H}-6 \mathrm{~B})$, $3.69\left(1 \mathrm{H}, \mathrm{d}, J=13.5 \mathrm{~Hz}, \mathrm{H}-\mathrm{1}^{\prime \prime} \mathrm{B}\right), 3.85\left(1 \mathrm{H}, \mathrm{q}, J=6.8 \mathrm{~Hz}, \mathrm{H}-1^{\prime}\right), 3.93(1 \mathrm{H}, \mathrm{d}, J=12.1 \mathrm{~Hz}, \mathrm{H}-8 \mathrm{~A}), 4.17(1 \mathrm{H}$, $\mathrm{dd}, J=12.1$ and $2.9 \mathrm{~Hz}, \mathrm{H}-8 \mathrm{~B}), 5.22\left(1 \mathrm{H}, \mathrm{d}, J=2 \mathrm{~Hz}, \mathrm{H}-1^{\prime \prime \prime}\right), 7.1-7.6(15 \mathrm{H}, \mathrm{Ar}-\mathrm{H}) .{ }^{13} \mathrm{C}-\mathrm{NMR} \delta(\mathrm{ppm})$ $\left(\mathrm{CDCl}_{3}\right): 12.6\left(\mathrm{CH}_{3}, \mathrm{C}-2^{\prime}\right), 18.9\left(\mathrm{CH},\left(\mathrm{CH}_{3}\right)_{2}\right), 25.9\left(\mathrm{CH}_{2}, \mathrm{C}-3\right), 29.5\left(\mathrm{CH},\left(\mathrm{CH}_{3}\right)_{2}\right), 30.1\left(\mathrm{CH}_{2}, \mathrm{C}-4\right), 35.2$ (CH, C-5), $43.2(\mathrm{CH}, \mathrm{C}-1), 46.6(\mathrm{CH}, \mathrm{C}-7), 49.6\left(\mathrm{CH}, \mathrm{C}-1^{\prime \prime}\right), 56.0\left(\mathrm{CH}, \mathrm{C}-1^{\prime}\right), 62.5(\mathrm{CH}, \mathrm{C}-2), 64.2\left(\mathrm{CH}_{2}\right.$, $\mathrm{C}-8), 66.0\left(\mathrm{CH}_{2}, \mathrm{C}-6\right), 73.4\left(\mathrm{CH}, \mathrm{C}-1^{\prime \prime \prime}\right), 99.0\left(\mathrm{CH},\left(\mathrm{CH}_{3}\right)_{2}\right), 125.6-129.2(\mathrm{CHx} 15, \mathrm{Ar}), 140.0\left(\mathrm{C}, \mathrm{C}_{i p s o}\right)$, $141.0\left(\mathrm{C}, \mathrm{C}_{\text {ipso }}\right), 144.2\left(\mathrm{C}, \mathrm{C}_{\text {ipso }}\right), 143.4\left(\mathrm{C}, \mathrm{C}_{\text {ipso }}\right)$.

To a solution of $4(25 \mathrm{mg}, 0.04 \mathrm{mmol})$ in $\mathrm{CH}_{2} \mathrm{Cl}_{2}(3.5 \mathrm{~mL})$, molecular sieves $(11 \mathrm{mg})$ and TPAP $(1 \mathrm{mg}, 0.004 \mathrm{mmol})$ were added. After $3 \mathrm{~h}$ of stirring, the resulting mixture was filtered through Celite-silica with $\mathrm{CH}_{2} \mathrm{Cl}_{2}$ and, after solvent evaporation, $23 \mathrm{mg}$ of $\mathbf{1 0}$ (yield $91 \%$ ) were obtained. 
Pentan-3-yl (1R,2R,5S)-2-(benzyl((R)-1-phenylethyl)amino)-5-((R)-1,3-dioxo-1-(pentan-3-yloxy)-3phenyl-propan-2-yl)cyclopentane-1-carboxylate (10): H.R.M.S.: calcd for $\mathrm{C}_{40} \mathrm{H}_{51} \mathrm{NO}_{5}$ : 626.3767; found: 626.3903. ${ }^{1} \mathrm{H}-\mathrm{NMR} \delta(\mathrm{ppm})\left(400 \mathrm{MHz}, \mathrm{CDCl}_{3}\right): 0.70\left(3 \mathrm{H}, \mathrm{t}, J=7.3 \mathrm{~Hz}, \mathrm{CH}\left(\mathrm{CH}_{2} \mathrm{CH}_{3}\right)_{2}\right), 0.77(3 \mathrm{H}, \mathrm{t}, J=$ $\left.7.3 \mathrm{~Hz}, \mathrm{CH}\left(\mathrm{CH}_{2} \mathrm{CH}_{3}\right)_{2}\right), 0.84\left(3 \mathrm{H}, \mathrm{t}, J=7.3 \mathrm{~Hz}, \mathrm{CH}\left(\mathrm{CH}_{2} \mathrm{CH}_{3}\right)_{2}\right), 0.92\left(3 \mathrm{H}, \mathrm{t}, J=7.3 \mathrm{~Hz}, \mathrm{CH}\left(\mathrm{CH}_{2} \mathrm{CH}_{3}\right)_{2}\right)$, $1.25\left(2 \mathrm{H}, \mathrm{m}, \mathrm{CH}\left(\mathrm{CH}_{2} \mathrm{CH}_{3}\right)_{2}\right), 1.31\left(3 \mathrm{H}, \mathrm{d}, J=6.9 \mathrm{~Hz}, \mathrm{H}-2^{\prime}\right) 1.41\left(2 \mathrm{H}, \mathrm{m}, \mathrm{CH}\left(\mathrm{CH}_{2} \mathrm{CH}_{3}\right)_{2}\right), 1.45(2 \mathrm{H}, \mathrm{m}$, $\left.\mathrm{CH}\left(\mathrm{CH}_{2} \mathrm{CH}_{3}\right)_{2}\right), 1.52(1 \mathrm{H}, \mathrm{m}, \mathrm{H}-4 \mathrm{~A}), 1.64\left(2 \mathrm{H}, \mathrm{m}, \mathrm{CH}\left(\mathrm{CH}_{2} \mathrm{CH}_{3}\right)_{2}\right), 1.75(2 \mathrm{H}, \mathrm{m}, \mathrm{H}-3), 1.85(1 \mathrm{H}, \mathrm{m}, \mathrm{H}-4 \mathrm{~B})$, $2.77(1 \mathrm{H}, \mathrm{m}, \mathrm{H}-5), 2.92(1 \mathrm{H}, \mathrm{dd}, J=8.6$ and $7.3 \mathrm{~Hz}, \mathrm{H}-1), 3.62(1 \mathrm{H}, \mathrm{m}, \mathrm{H}-2), 3.89(1 \mathrm{H}, \mathrm{q}, J=6.9 \mathrm{~Hz}$, H-1'), $4.3(1 \mathrm{H}, \mathrm{d}, J=10 \mathrm{~Hz}, \mathrm{H}-7), 3.90\left(1 \mathrm{H}, \mathrm{d}, J=14.5 \mathrm{~Hz}, \mathrm{H}-1^{\prime \prime} \mathrm{A}\right), 3.93\left(1 \mathrm{H}, \mathrm{d}, J=14.5 \mathrm{~Hz} \mathrm{H}-1^{\prime \prime} \mathrm{B}\right), 4.59$ $\left(1 \mathrm{H}\right.$, quintet, $\left.J=5.7 \mathrm{~Hz}, \mathrm{CH}\left(\mathrm{CH}_{2} \mathrm{CH}_{3}\right)_{2}\right), 4.69\left(1 \mathrm{H}\right.$, quintet, $\left.J=5.7 \mathrm{~Hz}, \mathrm{CH}\left(\mathrm{CH}_{2} \mathrm{CH}_{3}\right)_{2}\right)$, 7.1-7.6 $(13 \mathrm{H}$, $\mathrm{m}, \mathrm{Ar}-\mathrm{H}), 7.9(2 \mathrm{H}, \mathrm{d}, J=7.3 \mathrm{~Hz}) .{ }^{13} \mathrm{C}-\mathrm{NMR} \delta(\mathrm{ppm})\left(\mathrm{CDCl}_{3}\right): 9.5\left(\mathrm{CH}_{3}, \mathrm{CH}\left(\mathrm{CH}_{2} \mathrm{CH}_{3}\right)_{2} \times 3\right), 9.6\left(\mathrm{CH}_{3}\right.$, $\left.\mathrm{CH}\left(\mathrm{CH}_{2} \mathrm{CH}_{3}\right)_{2}\right) 15.8\left(\mathrm{CH}_{3}, \mathrm{C}-2^{\prime}\right), 25.5\left(\mathrm{CH}_{2}, \mathrm{CH}\left(\mathrm{CH}_{2} \mathrm{CH}_{3}\right)_{2}\right), 25.6\left(\mathrm{CH}_{2}, \mathrm{CH}\left(\mathrm{CH}_{2} \mathrm{CH}_{3}\right)_{2} \times 2\right), 26.1\left(\mathrm{CH}_{2}\right.$, $\left.\mathrm{CH}\left(\mathrm{CH}_{2} \mathrm{CH}_{3}\right)_{2}\right), 27.7\left(\mathrm{CH}_{2}, \mathrm{C}-3\right), 28.8\left(\mathrm{CH}_{2}, \mathrm{C}-4\right), 41.5(\mathrm{CH}, \mathrm{C}-5), 52.6(\mathrm{CH}, \mathrm{C}-1), 50.3\left(\mathrm{CH}_{2}, \mathrm{C}-1^{\prime \prime}\right), 57.0$ $\left(\mathrm{CH}_{2}, \mathrm{C}-7\right), 57.8\left(\mathrm{CH}, \mathrm{C}-1^{\prime}\right), 65.3(\mathrm{CH}, \mathrm{C}-2), 77.1\left(\mathrm{CH}, \mathrm{CH}\left(\mathrm{CH}_{2} \mathrm{CH}_{3}\right)_{2}\right), 78.5\left(\mathrm{CH}, \mathrm{CH}\left(\mathrm{CH}_{2} \mathrm{CH}_{3}\right)_{2}\right), 126.7-$ 128.8 (CHx15, Ar), 137.8 (C, $\left.\mathrm{C}_{\mathrm{ipso}}\right), 141.9$ (C, $\mathrm{C}_{\mathrm{ipso}}$ ), 144.7 (C, $\left.\mathrm{C}_{\mathrm{ipso}}\right), 169.2$ (COOR, C-8), 175.1 (COOR, C-6), 194.1 (C, C-1'").

To a solution of $4+3(29 \mathrm{mg}, 0.045 \mathrm{mmol})$ in $\mathrm{CH}_{2} \mathrm{Cl}_{2}(3 \mathrm{~mL})$ PDC (26 mg, $\left.0.0687 \mathrm{mmol}\right)$ was added. After $24 \mathrm{~h}$, saturated $\mathrm{NH}_{4} \mathrm{Cl}$ solution $(3 \mathrm{~mL}$ ) was added and the resulting mixture was filtered, dissolved in EtOAc and washed with $\mathrm{H}_{2} \mathrm{O}$ and brine. After drying over $\mathrm{Na}_{2} \mathrm{SO}_{4}$, filteration and solvent evaporated, the resulting $27 \mathrm{mg}$ were chromatographed and the desired compounds eluted with hexane/EtOAc $95: 5$ to afford $5 \mathrm{mg}$ of $\mathbf{1 0}$ (yield 18\%) and $13 \mathrm{mg}$ of $\mathbf{1 1}$ (yield $49 \%$ ).

Pentan-3-yl (1R,2S,5R)-2-((R)-1,3-dioxo-1-(pentan-3-yloxy)-3-phenylpropan-2-yl)-5-(N-((R)-1phenyl-ethyl)benzamido)cyclopentane-1-carboxylate (11) IR $\left(\mathrm{cm}^{-1}\right)$ : 2969, 1732, 1688, 1456, 1219, 1105, 702. ${ }^{1} \mathrm{H}-\mathrm{NMR} \delta(\mathrm{ppm})\left(400 \mathrm{MHz}, \mathrm{CDCl}_{3}\right): 0.60-0.93\left(12 \mathrm{H}, \mathrm{m}, \mathrm{CH}\left(\mathrm{CH}_{2} \mathrm{CH}_{3}\right)_{2}\right), 1.26(3 \mathrm{H}, \mathrm{d}, J=7.1$ $\left.\mathrm{Hz}, \mathrm{H}-2^{\prime}\right), 1.30-1.62\left(8 \mathrm{H}, \mathrm{m}, \mathrm{CH}\left(\mathrm{CH}_{2} \mathrm{CH}_{3}\right)_{2}\right), 1.52-1.99(4 \mathrm{H}, \mathrm{m}, \mathrm{H}-3$ and $\mathrm{H}-4), 2.48(1 \mathrm{H}, \mathrm{dd}, J=8.6$ and $7.3 \mathrm{~Hz}, \mathrm{H}-1), 3.15$ (1H, m, H-5), 3.17 (1H, m, H-7), 3.89 (1H, q, J = 7.1 Hz, H-1' ), 4.45 (1H, dd, J = 13.3 and $7.3 \mathrm{~Hz}, \mathrm{H}-2), 4.71\left(2 \mathrm{H}, \mathrm{m}, \mathrm{CH}\left(\mathrm{CH}_{2} \mathrm{CH}_{3}\right)_{2} \times 2\right), 7.1-7.6(11 \mathrm{H}, \mathrm{m}, \mathrm{Ar}-\mathrm{H}), 7.9(4 \mathrm{H}, \mathrm{m}, \mathrm{Ar}-\mathrm{H}) .{ }^{13} \mathrm{C}-\mathrm{NMR}$ $\delta(\mathrm{ppm})\left(\mathrm{CDCl}_{3}\right)$ : $9.4\left(\mathrm{CH}_{3}, \mathrm{CH}\left(\mathrm{CH}_{2} \mathrm{CH}_{3}\right)_{2}\right) 9.5\left(\mathrm{CH}_{3}, \mathrm{CH}\left(\mathrm{CH}_{2} \mathrm{CH}_{3}\right)_{2} \times 2\right)$, $9.7\left(\mathrm{CH}_{3}, \mathrm{CH}\left(\mathrm{CH}_{2} \mathrm{CH}_{3}\right)_{2}\right)$, 22.2 $\left(\mathrm{CH}_{3}, \mathrm{C}-2^{\prime}\right), 25.7\left(\mathrm{CH}_{2}, \mathrm{CH}\left(\mathrm{CH}_{2} \mathrm{CH}_{3}\right)_{2}\right), 26.0\left(\mathrm{CH}_{2}, \mathrm{CH}\left(\mathrm{CH}_{2} \mathrm{CH}_{3}\right)_{2}\right), 26.1\left(\mathrm{CH}_{2}, \mathrm{CH}\left(\mathrm{CH}_{2} \mathrm{CH}_{3}\right)_{2}\right)$, $26.2\left(\mathrm{CH}_{2}, \mathrm{CH}\left(\mathrm{CH}_{2} \mathrm{CH}_{3}\right)_{2}\right), 29.5\left(\mathrm{CH}_{2}, \mathrm{C}-3\right), 31.1\left(\mathrm{CH}_{2}, \mathrm{C}-4\right), 43.3(\mathrm{CH}, \mathrm{C}-5), 53.1(\mathrm{CH}, \mathrm{C}-1), 57.9\left(\mathrm{CH}_{2}\right.$, C-7), $58.1\left(\mathrm{CH}, \mathrm{C}-1^{\prime}\right), 61.5(\mathrm{CH}, \mathrm{C}-2), 78.1\left(\mathrm{CH}, \mathrm{CH}\left(\mathrm{CH}_{2} \mathrm{CH}_{3}\right)_{2}\right), 78.5\left(\mathrm{CH}, \mathrm{CH}\left(\mathrm{CH}_{2} \mathrm{CH}_{3}\right)_{2}\right), 128.8-129.5$ (CHx15, Ar), 133.7 (C, $\left.\mathrm{C}_{\mathrm{ipso}}\right), 133.8$ (C, $\left.\mathrm{C}_{\mathrm{ipso}}\right), 136.6$ (C, $\left.\mathrm{C}_{\mathrm{ipso}}\right), 168.9$ (COOR, C-8), 173.7 (COOR, C-6), 194.7 (C, C-1'"'), 195.3 (C, C-1' $\left.{ }^{\prime \prime}\right)$.

\subsection{General Tandem Domino Michael Addition Procedure: Compounds 12-20}

To a solution of $(R)-\mathbf{1}$ (2.3 equivalents) in THF at $-78{ }^{\circ} \mathrm{C}$ under $\mathrm{Ar}$ atmosphere, $1.6 \mathrm{M} n$-BuLi (2.2 equivalents) was added. After $50 \mathrm{~min}$ of reaction, 2 ( 1 equivalent) was added and, after $1 \mathrm{~h}$ of reaction, an electrophile (1.5 equivalents) in THF was added. Then, after $3 \mathrm{~h}$, the reaction was quenched with $\mathrm{NH}_{4} \mathrm{Cl}$. The resulting reaction mixture was dissolved with EtOAc and washed with $10 \%$ aqueous citric acid solution, $\mathrm{H}_{2} \mathrm{O}$ and brine. After being dried over $\mathrm{Na}_{2} \mathrm{SO}_{4}$ and filtered, the solvent was evaporated and the reaction mixture was chromatographed.

When using benzaldehyde as electrophile, $1.34 \mathrm{~g}$ of reaction mixture were obtained and chromatographed to give $63 \mathrm{mg}$ (yield 30\%) of $\mathbf{1 2}$ and $57 \mathrm{mg}$ (yield $27 \%$ ) of $\mathbf{1 3 .}$

Pentan-3-yl (1R,2R,5S)-2-(benzyl((R)-1-phenylethyl)amino)-5-((2R,3R,E)-3-hydroxy-1-oxo-1(pentan-3-yl-oxy)-5-phenylpent-4-en-2-yl)cyclopentane-1-carboxylate (12): IR ( $\left.\mathrm{cm}^{-1}\right)$ : 3503, 2967, 1724, 1458, 1177, 1105, 698. H.R.M.S.: calcd for $\mathrm{C}_{42} \mathrm{H}_{55} \mathrm{NO}_{5}$ : 653.4080; found: $653.4150 .{ }^{1} \mathrm{H}-\mathrm{NMR} \delta$ (ppm) $\left(200 \mathrm{MHz}, \mathrm{CDCl}_{3}\right): 0.60-0.96\left(9 \mathrm{H}, \mathrm{m}, \mathrm{CH}\left(\mathrm{CH}_{2} \mathrm{CH}_{3}\right)_{2}\right), 1.12\left(3 \mathrm{H}, \mathrm{m}, \mathrm{CH}\left(\mathrm{CH}_{2} \mathrm{CH}_{3}\right)_{2}\right), 1.32(3 \mathrm{H}, \mathrm{d}, J=6.8$ $\left.\mathrm{Hz}, \mathrm{H}-2^{\prime}\right), 1.35-1.58\left(8 \mathrm{H}, \mathrm{m}, \mathrm{CH}\left(\mathrm{CH}_{2} \mathrm{CH}_{3}\right)_{2}\right), 1.55-1.90(4 \mathrm{H}, \mathrm{m}, \mathrm{H}-3$ and $\mathrm{H}-4), 2.53(1 \mathrm{H}, \mathrm{d}, J=7.5, \mathrm{H}-7)$, $2.61(1 \mathrm{H}, \mathrm{m}, \mathrm{H}-5), 2.82(1 \mathrm{H}, \mathrm{t}, J=6 \mathrm{~Hz}, \mathrm{H}-1), 3.35(1 \mathrm{H}, \mathrm{d}, J=8.4 \mathrm{~Hz}, \mathrm{OH}), 3.63\left(1 \mathrm{H}, \mathrm{d}, J=4.2 \mathrm{~Hz}, \mathrm{H}-1^{\prime \prime} \mathrm{A}\right)$, $3.77(1 \mathrm{H}, \mathrm{ddd}, \mathrm{H}-2), 3.85\left(1 \mathrm{H}, \mathrm{q}, J=6.8 \mathrm{~Hz}, \mathrm{H}-1^{\prime}\right), 3.87\left(1 \mathrm{H}, \mathrm{d}, J=4.2 \mathrm{~Hz}, \mathrm{H}-1^{\prime \prime} \mathrm{B}\right), 4.42\left(1 \mathrm{H}, \mathrm{m}, \mathrm{H}-1^{\prime \prime \prime}\right)$, $4.55\left(1 \mathrm{H}, \mathrm{q}, J=6.0, \mathrm{CH}\left(\mathrm{CH}_{2} \mathrm{CH}_{3}\right)_{2}\right), 4.71\left(1 \mathrm{H}\right.$, quintet, $\left.J=6.0 \mathrm{~Hz}, \mathrm{CH}\left(\mathrm{CH}_{2} \mathrm{CH}_{3}\right)_{2}\right), 6.18(1 \mathrm{H}, \mathrm{dd}, J=14.2$ 
and $\left.5.2 \mathrm{~Hz}, \mathrm{H}-2^{\prime \prime \prime}\right), 6.60\left(1 \mathrm{H}, \mathrm{d}, J=17.4 \mathrm{~Hz}, \mathrm{H}-3^{\prime \prime \prime}\right), 7.1-7.6(10 \mathrm{H}, \mathrm{Ar}-\mathrm{H}) .{ }^{13} \mathrm{C}-\mathrm{NMR} \delta(\mathrm{ppm})\left(\mathrm{CDCl}_{3}\right): 9.4$ $\left(\mathrm{CH}_{3}, \mathrm{CH}\left(\mathrm{CH}_{2} \mathrm{CH}_{3}\right)_{2} \times 2\right), 9.8\left(\mathrm{CH}_{3}, \mathrm{CH}\left(\mathrm{CH}_{2} \mathrm{CH}_{3}\right)_{2} \times 2\right), 15.6\left(\mathrm{CH}_{3}, \mathrm{C}-2^{\prime}\right), 25.5\left(\mathrm{CH}_{2}, \mathrm{CH}\left(\mathrm{CH}_{2} \mathrm{CH}_{3}\right)_{2}\right)$, $25.9\left(\mathrm{CH}_{2}, \mathrm{CH}\left(\mathrm{CH}_{2} \mathrm{CH}_{3}\right)_{2}\right), 26.1\left(\mathrm{CH}_{2}, \mathrm{CH}\left(\mathrm{CH}_{2} \mathrm{CH}_{3}\right)_{2}\right), 27.5\left(\mathrm{CH}_{2}, \mathrm{CH}\left(\mathrm{CH}_{2} \mathrm{CH}_{3}\right)_{2}\right), 28.8\left(\mathrm{CH}_{2}, \mathrm{C}-3\right)$, $29.1\left(\mathrm{CH}_{2}, \mathrm{C}-4\right), 41.5(\mathrm{CH}, \mathrm{C}-5), 50.3\left(\mathrm{CH}_{2}, \mathrm{C}-1^{\prime \prime}\right), 52.5(\mathrm{CH}, \mathrm{C}-1), 53.8\left(\mathrm{CH}_{2}, \mathrm{C}-7\right), 57.5\left(\mathrm{CH}, \mathrm{C}-1^{\prime}\right), 65.1$ $(\mathrm{CH}, \mathrm{C}-2), 71.1\left(\mathrm{CH}, \mathrm{C}-1^{\prime \prime \prime}\right), 76.8\left(\mathrm{CH}, \mathrm{CH}\left(\mathrm{CH}_{2} \mathrm{CH}_{3}\right)_{2}\right), 77.1\left(\mathrm{CH}, \mathrm{CH}\left(\mathrm{CH}_{2} \mathrm{CH}_{3}\right)_{2}\right)$, 126.6-128.8 (CHx15, $\mathrm{Ar}), 130.4(\mathrm{CH}, \mathrm{Ph}-\mathrm{CH}=\mathrm{CH}-), 130.9(\mathrm{CH}, \mathrm{Ph}-\mathrm{CH}=\mathrm{CH}-), 136.7$ (C, $\left.\mathrm{C}_{\mathrm{ipso}}\right), 141.6\left(\mathrm{C}, \mathrm{C}_{\mathrm{ipso}}\right), 144.3\left(\mathrm{C}, \mathrm{C}_{\mathrm{ipso}}\right)$, 174.2 (COOR, C-8), 174.9 (COOR, C-6).

Pentan-3-yl (1R,2R,5S)-2-(benzyl((R)-1-phenylethyl)amino)-5-((2R,3S,E)-3-hydroxy-1-oxo-1(pentan-3-yl-oxy)-5-phenylpent-4-en-2-yl)cyclopentane-1-carboxylate (13): IR ( $\left.\mathrm{cm}^{-1}\right)$ : 3510, 2969, 1719, 1458, 1105, 698. ${ }^{1} \mathrm{H}-\mathrm{NMR} \delta$ (ppm) $\left(200 \mathrm{MHz}, \mathrm{CDCl}_{3}\right): 0.63\left(3 \mathrm{H}, \mathrm{m}, \mathrm{CH}\left(\mathrm{CH}_{2} \mathrm{CH}_{3}\right)_{2}\right), 0.73-1.01(9 \mathrm{H}, \mathrm{m}$, $\left.\mathrm{CH}\left(\mathrm{CH}_{2} \mathrm{CH}_{3}\right)_{2}\right), 1.27\left(3 \mathrm{H}, \mathrm{d}, J=6.8 \mathrm{~Hz}, \mathrm{H}-2^{\prime}\right), 1.38-1.95\left(8 \mathrm{H}, \mathrm{m}, \mathrm{CH}\left(\mathrm{CH}_{2} \mathrm{CH}_{3}\right)_{2} ; 2 \mathrm{H}, \mathrm{m}, \mathrm{H}-3 ; 2 \mathrm{H}, \mathrm{m}\right.$, $\mathrm{H}-4), 2.60(1 \mathrm{H}, \mathrm{m}, \mathrm{H}-5), 2.63(1 \mathrm{H}, \mathrm{d}, J=8 \mathrm{~Hz}, \mathrm{H}-7), 2.93(1 \mathrm{H}, \mathrm{d}, J=4.5 \mathrm{~Hz}, \mathrm{OH}), 3.10(1 \mathrm{H}, \mathrm{t}, J=6 \mathrm{~Hz}$, $\mathrm{H}-1), 3.63\left(1 \mathrm{H}, \mathrm{d}, J=4.2 \mathrm{~Hz}, \mathrm{H}-1^{\prime \prime} \mathrm{A}\right), 3.77(1 \mathrm{H}, \mathrm{m}, \mathrm{H}-2), 3.85\left(1 \mathrm{H}, \mathrm{q}, J=6.8 \mathrm{~Hz}, \mathrm{H}-1^{\prime}\right), 3.87(1 \mathrm{H}, \mathrm{d}, J=$ $\left.6.8 \mathrm{~Hz}, \mathrm{H}-1^{\prime \prime} \mathrm{B}\right), 4.31\left(1 \mathrm{H}, \mathrm{m}, \mathrm{H}-1^{\prime \prime \prime}\right), 4.64\left(2 \mathrm{H}, \mathrm{m}, \mathrm{CH}\left(\mathrm{CH}_{2} \mathrm{CH}_{3}\right)_{2} \times 2\right), 6.18\left(1 \mathrm{H}, \mathrm{dd}, \mathrm{H}-2^{\prime \prime \prime}\right), 6.53(1 \mathrm{H}, \mathrm{d}$, H-3'"), 7.1-7.6 (10H, Ar-H).

When using formaldehyde as electrophile, $155 \mathrm{mg}$ of crude product were obtained and chromatographed to give after elution with hexane/EtOAc 95:5 $140 \mathrm{mg}$ (yield 80\%) of $\mathbf{1 4}$.

Pentan-3-yl (1R,2R,5S)-2-(benzyl((R)-1-phenylethyl)amino)-5-((S)-3-hydroxy-1-oxo-1-(pentan-3yloxy)-propan-2-yl)cyclopentane-1-carboxylate (14): IR ( $\left.\mathrm{cm}^{-1}\right)$ : 3488, 2969, 1726, 1456, 1105, 748, 700. H.R.M.S.: calcd for $\mathrm{C}_{34} \mathrm{H}_{49} \mathrm{NO}_{5}$ : 551.3611; found: 551.3573 . ${ }^{1} \mathrm{H}-\mathrm{NMR} \delta$ (ppm) $\left(400 \mathrm{MHz}, \mathrm{CDCl}_{3}\right): 0.83$ $\left(9 \mathrm{H}, \mathrm{m}, \mathrm{CH}\left(\mathrm{CH}_{2} \mathrm{CH}_{3}\right)_{2}\right), 0.96\left(3 \mathrm{H}, \mathrm{t}, J=7.5 \mathrm{~Hz}, \mathrm{CH}\left(\mathrm{CH}_{2} \mathrm{CH}_{3}\right)_{2}\right), 1.30\left(3 \mathrm{H}, \mathrm{d}, J=6.8 \mathrm{~Hz}, \mathrm{H}-2^{\prime}\right), 1.4-1.6$ $\left(8 \mathrm{H}, \mathrm{m}, \mathrm{CH}\left(\mathrm{CH}_{2} \mathrm{CH}_{3}\right)_{2}\right), 1.63(2 \mathrm{H}, \mathrm{m}, \mathrm{H}-3), 1.72(2 \mathrm{H}, \mathrm{m}, \mathrm{H}-4), 2.46(1 \mathrm{H}, \mathrm{m}, \mathrm{H}-5), 2.62(1 \mathrm{H}, \mathrm{d}, J=7.5$, $\mathrm{H}-7), 2.76(1 \mathrm{H}, \mathrm{t}, J=9.5 \mathrm{~Hz}, \mathrm{H}-1), 3.60\left(1 \mathrm{H}, \mathrm{m}, \mathrm{H}-1^{\prime \prime \prime} \mathrm{A}\right), 3.64(1 \mathrm{H}, \mathrm{m}, \mathrm{H}-2), 3.65\left(1 \mathrm{H}, \mathrm{m}, \mathrm{H}-1^{\prime \prime \prime} \mathrm{B}\right), 3.71(1 \mathrm{H}$, $\left.\mathrm{d}, J=11.1 \mathrm{~Hz}, \mathrm{H}-1^{\prime \prime} \mathrm{A}\right), 3.64(1 \mathrm{H}, \mathrm{m}, \mathrm{H}-2), 3.80\left(1 \mathrm{H}, \mathrm{d}, J=11.1 \mathrm{~Hz}, \mathrm{H}-1^{\prime \prime} \mathrm{B}\right), 3.85\left(1 \mathrm{H}, \mathrm{q}, J=6.8 \mathrm{~Hz}, \mathrm{H}-1^{\prime}\right)$, $4.61\left(1 \mathrm{H}\right.$, quintet, $\left.J=6.0, \mathrm{CH}\left(\mathrm{CH}_{2} \mathrm{CH}_{3}\right)_{2}\right), 4.75\left(1 \mathrm{H}\right.$, quintet, $\left.J=6 \mathrm{~Hz}, \mathrm{CH}\left(\mathrm{CH}_{2} \mathrm{CH}_{3}\right)_{2}\right), 7.1-7.6(10 \mathrm{H}$, Ar-H). ${ }^{13} \mathrm{C}-\mathrm{NMR} \delta$ (ppm) $\left(\mathrm{CDCl}_{3}\right): 9.4\left(\mathrm{CH}_{3}, \mathrm{CH}\left(\mathrm{CH}_{2} \mathrm{CH}_{3}\right)_{2}\right), 9.5\left(\mathrm{CH}_{3}, \mathrm{CH}\left(\mathrm{CH}_{2} \mathrm{CH}_{3}\right)_{2}\right), 9.7\left(\mathrm{CH}_{3}\right.$, $\left.\mathrm{CH}\left(\mathrm{CH}_{2} \mathrm{CH}_{3}\right)_{2}\right), 9.8\left(\mathrm{CH}_{3}, \mathrm{CH}\left(\mathrm{CH}_{2} \mathrm{CH}_{3}\right)_{2}\right), 15.4\left(\mathrm{CH}_{3}, \mathrm{C}-2^{\prime}\right), 25.4\left(\mathrm{CH}_{2}, \mathrm{CH}\left(\mathrm{CH}_{2} \mathrm{CH}_{3}\right)_{2} \times 2\right), 25.6\left(\mathrm{CH}_{2}\right.$, C-3), $26.4\left(\mathrm{CH}_{2}, \mathrm{CH}\left(\mathrm{CH}_{2} \mathrm{CH}_{3}\right)_{2}\right), 26.6\left(\mathrm{CH}_{2}, \mathrm{CH}\left(\mathrm{CH}_{2} \mathrm{CH}_{3}\right)_{2}\right), 41.1(\mathrm{CH}, \mathrm{C}-5), 49.5(\mathrm{CH}, \mathrm{C}-1), 50.2\left(\mathrm{CH}_{2}\right.$, C-1" $)$, $51.8\left(\mathrm{CH}, \mathrm{C}-1^{\prime}\right), 57.8(\mathrm{CH}, \mathrm{C}-7), 61.1\left(\mathrm{CH}_{2}, \mathrm{C}-1^{\prime \prime \prime}\right), 64.5\left(\mathrm{CH}_{2}, \mathrm{C}-2\right), 77.1\left(\mathrm{CH}, \mathrm{CH}\left(\mathrm{CH}_{2} \mathrm{CH}_{3}\right)_{2}\right)$, $77.6\left(\mathrm{CH}, \mathrm{CH}\left(\mathrm{CH}_{2} \mathrm{CH}_{3}\right)_{2}\right), 126.4-128(\mathrm{CHx} 10, \mathrm{Ar}), 141.6\left(\mathrm{C}, \mathrm{C}_{\text {ipso }}\right), 144.3\left(\mathrm{C}, \mathrm{C}_{\mathrm{ipso}}\right), 173.9(\mathrm{COOR}, \mathrm{C}-8)$, 175.3 (COOR, C-6).

When using acetone as electrophile, $160 \mathrm{mg}$ were obtained and chromatographed to obtain $60 \mathrm{mg}$ (yield $27 \%$ ) of $\mathbf{1 5}$ and $100 \mathrm{mg}$ (yield $25 \%$ ) of $\mathbf{1 6}$.

Pentan-3-yl (1R,2R,5S)-2-(benzyl((R)-1-phenylethyl)amino)-5-((R)-3-hydroxy-3-methyl-1-oxo-1(pentan-3-yloxy)butan-2-yl)cyclopentane-1-carboxylate (15): ${ }^{1} \mathrm{H}-\mathrm{NMR} \delta$ (ppm) $\left(200 \mathrm{MHz}, \mathrm{CDCl}_{3}\right.$ ): 0.8-0.9 (12H, m, $\left.\mathrm{CH}\left(\mathrm{CH}_{2} \mathrm{CH}_{3}\right)_{2}\right), 1.17\left(3 \mathrm{H}, \mathrm{s}, \mathrm{CH}_{3}\right), 1.18\left(3 \mathrm{H}, \mathrm{s}, \mathrm{CH}_{3}\right), 1.26\left(3 \mathrm{H}, \mathrm{d}, J=6.8 \mathrm{~Hz}, \mathrm{H}-2^{\prime}\right)$, 1.41-1.91 (8H, m, CH $\left.\left.\left(\mathrm{CH}_{2} \mathrm{CH}_{3}\right)_{2}\right) ; 2 \mathrm{H}, \mathrm{m}, \mathrm{H}-3 ; 2 \mathrm{H}, \mathrm{m}, \mathrm{H}-4\right), 2.38(1 \mathrm{H}, \mathrm{d}, J=5.2 \mathrm{~Hz}, \mathrm{H}-7), 2.63(1 \mathrm{H}, \mathrm{m}$, $\mathrm{H}-5), 2.83(1 \mathrm{H}, \mathrm{t}, J=8.8 \mathrm{~Hz}, \mathrm{H}-1), 3.41-3.92\left(1 \mathrm{H}, \mathrm{m}, \mathrm{H}-\mathrm{1}^{\prime} ; 2 \mathrm{H}, \mathrm{m}, \mathrm{H}-1^{\prime \prime \prime} ; 1 \mathrm{H}, \mathrm{m}, \mathrm{H}-2\right), 4.50$ (1H, quintet, $\left.J=6 \mathrm{~Hz}, \mathrm{CH}\left(\mathrm{CH}_{2} \mathrm{CH}_{3}\right)_{2}\right), 4.75\left(1 \mathrm{H}\right.$, quintet, $\left.J=6 \mathrm{~Hz}, \mathrm{CH}\left(\mathrm{CH}_{2} \mathrm{CH}_{3}\right)_{2}\right), 7.1-7.6(10 \mathrm{H}, \mathrm{Ar}-\mathrm{H}) .{ }^{13} \mathrm{C}-\mathrm{NMR} \delta$ (ppm) $\left(\mathrm{CDCl}_{3}\right)$ : $9.3\left(\mathrm{CH}_{3}, \mathrm{CH}\left(\mathrm{CH}_{2} \mathrm{CH}_{3}\right)_{2} \times 2\right), 9.8\left(\mathrm{CH}_{3}, \mathrm{CH}\left(\mathrm{CH}_{2} \mathrm{CH}_{3}\right)_{2} \times 2\right), 14.7\left(\mathrm{CH}_{3}, \mathrm{C}-2^{\prime}\right), 24.7\left(\mathrm{CH}_{2}\right.$, $\left.\mathrm{CH}\left(\mathrm{CH}_{2} \mathrm{CH}_{3}\right)_{2} \times 2\right), 26.0\left(\mathrm{CH}_{2}, \mathrm{CH}\left(\mathrm{CH}_{2} \mathrm{CH}_{3}\right)_{2}\right), 26.5\left(\mathrm{CH}_{2}, \mathrm{C}-3\right), 26.6\left(\mathrm{CH}_{2}, \mathrm{CH}\left(\mathrm{CH}_{2} \mathrm{CH}_{3}\right)_{2}\right), 30.3\left(\mathrm{CH}_{2}\right.$, $\mathrm{C}-4), 39.0(\mathrm{CH}, \mathrm{C}-5), 49.5(\mathrm{CH}, \mathrm{C}-1), 50.1\left(\mathrm{CH}_{2}, \mathrm{C}-1^{\prime \prime}\right), 52.1(\mathrm{CH}, \mathrm{C}-1), 57.8(\mathrm{CH}, \mathrm{C}-7), 61.1\left(\mathrm{CH}_{2}, \mathrm{C}-1^{\prime \prime \prime}\right)$, $64.5\left(\mathrm{CH}_{2}, \mathrm{C}-2\right), 77.1\left(\mathrm{CH}, \mathrm{CH}\left(\mathrm{CH}_{2} \mathrm{CH}_{3}\right)_{2}\right), 77.6\left(\mathrm{CH}, \mathrm{CH}\left(\mathrm{CH}_{2} \mathrm{CH}_{3}\right)_{2}\right), 126.4-128(\mathrm{CHx} 10, \mathrm{Ar}), 141.6(\mathrm{C}$, $\left.\mathrm{C}_{\mathrm{ipso}}\right), 144.3\left(\mathrm{C}, \mathrm{C}_{\mathrm{ipso}}\right), 173.9$ (COOR, C -8$), 175.3$ (COOR, C-6).

Pentan-3-yl (1R,2R,5R)-2-(benzyl((R)-1-phenylethyl)amino)-5-(2-oxo-2-(pentan-3-yloxy)ethyl) cyclopentane-1-carboxylate (16): $[\alpha]_{\mathrm{D}}{ }^{26}=-30.1\left(\mathrm{CHCl}_{3}, \mathrm{c}=1.55\right) . \mathrm{IR}\left(\mathrm{cm}^{-1}\right): 2969,1728,1454,1170,974$, 698. ${ }^{1} \mathrm{H}-\mathrm{NMR} \delta(\mathrm{ppm})\left(400 \mathrm{MHz}, \mathrm{CDCl}_{3}\right): 0.78\left(3 \mathrm{H}, \mathrm{t}, J=7.5 \mathrm{~Hz}, \mathrm{CH}\left(\mathrm{CH}_{2} \mathrm{CH}_{3}\right)_{2}\right), 0.85(6 \mathrm{H}, \mathrm{t}, J=7.5$, $\left.\mathrm{CH}\left(\mathrm{CH}_{2} \mathrm{CH}_{3}\right)_{2} \times 2\right), 0.94\left(3 \mathrm{H}, \mathrm{t}, J=7.5 \mathrm{~Hz}, \mathrm{CH}\left(\mathrm{CH}_{2} \mathrm{CH}_{3}\right)_{2}\right), 1.28\left(3 \mathrm{H}, \mathrm{d}, J=6.8, \mathrm{H}-2^{\prime}\right), 1.40-1.75(10 \mathrm{H}, \mathrm{m}$, $\left.\mathrm{CH}_{2}\right), 1.75$ - $1.85(2 \mathrm{H}, \mathrm{m}), 2.17(1 \mathrm{H}, \mathrm{dd}, J=14.8$ and $9.6 \mathrm{~Hz}, \mathrm{H}-7 \mathrm{~A}), 2.39(1 \mathrm{H}, \mathrm{m}, \mathrm{H}-5), 2.50(1 \mathrm{H}, \mathrm{dd}, J=$ 9.6 and $3.6 \mathrm{~Hz}, \mathrm{H}-7 \mathrm{~B}), 2.53(1 \mathrm{H}, \mathrm{dd}, J=10.0$ and $10.0 \mathrm{~Hz}, \mathrm{H}-1), 3.71(1 \mathrm{H}, \mathrm{m}, \mathrm{H}-2), 3.75\left(2 \mathrm{H}, \mathrm{m}, \mathrm{H}-1^{\prime \prime}\right)$, 
$3.86\left(1 \mathrm{H}, \mathrm{q}, J=6.8 \mathrm{~Hz}, \mathrm{H}-1^{\prime}\right), 4.58\left(1 \mathrm{H}\right.$, quintet, $\left.J=7.5 \mathrm{~Hz}, \mathrm{CH}\left(\mathrm{CH}_{2} \mathrm{CH}_{3}\right)_{2}\right), 4.71(1 \mathrm{H}$, quintet, $J=7.5$ $\left.\mathrm{Hz}, \mathrm{CH}\left(\mathrm{CH}_{2} \mathrm{CH}_{3}\right)_{2}\right), 7.1-7.5(10, \mathrm{~m}, \mathrm{Ar}-\mathrm{H}) .{ }^{13} \mathrm{C}-\mathrm{NMR} \delta(\mathrm{ppm})\left(\mathrm{CDCl}_{3}\right): 9.4\left(\mathrm{CH}_{3}, \mathrm{CH}\left(\mathrm{CH}_{2} \mathrm{CH}_{3}\right)_{2}\right), 9.5$ $\left(\mathrm{CH}_{3}, \mathrm{CH}\left(\mathrm{CH}_{2} \mathrm{CH}_{3}\right)_{2} \times 3\right), 15.5\left(\mathrm{CH} 3, \mathrm{C}-2^{\prime}\right), 25.8\left(\mathrm{CH}_{2}, \mathrm{C}-3\right), 26.4\left(\mathrm{CH}_{2}, \mathrm{CH}\left(\mathrm{CH}_{2} \mathrm{CH}_{3}\right)_{2} \times 3\right), 26.7\left(\mathrm{CH}_{2}\right.$, $\left.\mathrm{CH}\left(\mathrm{CH} 2 \mathrm{CH}_{3}\right)_{2}\right), 31.0\left(\mathrm{CH}_{2}, \mathrm{C}-4\right), 39.0\left(\mathrm{CH}_{2}, \mathrm{C}-7\right), 39.1(\mathrm{CH}, \mathrm{C}-5), 50.0\left(\mathrm{CH}_{2}, \mathrm{C}-1^{\prime \prime}\right), 55.3(\mathrm{CH}, \mathrm{C}-1), 58.0$ $\left(\mathrm{CH}, \mathrm{C}-1^{\prime}\right), 63.7(\mathrm{CH}, \mathrm{C}-2), 76.6\left(\mathrm{CH}, \mathrm{CH}\left(\mathrm{CH}_{2} \mathrm{CH}_{3}\right)_{2} \times 2\right), 126.5$ - $128.5(\mathrm{CHx} 10, \mathrm{Ar}), 141.6\left(\mathrm{C}, \mathrm{C}_{i p s o}\right), 144.3$ (C, $\left.\mathrm{C}_{i p s o}\right), 171.7$ (COOR, C-8), 174.3 (COOR, C-6).

When using diphenylketone as electrophile, $483 \mathrm{mg}$ of crude product were obtained and chromatographed. The desired compounds were eluted with hexane/EtOAc 95:5 to afford $79 \mathrm{mg}$ (yield $52 \%$ ) of $\mathbf{1 7}$ and $99 \mathrm{mg}$ (yield $15 \%$ ) of $\mathbf{1 6 .}$.

Pentan-3-yl (1R,2R,5S)-2-(benzyl((R)-1-phenylethyl)amino)-5-((R)-1-hydroxy-3-oxo-3-(pentan-3yloxy)-1,1-diphenylpropan-2-yl)cyclopentane-1-carboxylate (17): IR $\left(\mathrm{cm}^{-1}\right): 3472,2969,1728,1192$, 1103, 746, 702. H.R.M.S.: calcd for $\mathrm{C}_{46} \mathrm{H}_{57} \mathrm{NO}_{5}$ : 703.4237; found: 704.4325. ${ }^{1} \mathrm{H}-\mathrm{NMR} \delta$ (ppm) $(400 \mathrm{MHz}$, $\left.\mathrm{CHCl}_{3}\right): 0.66\left(3 \mathrm{H}, \mathrm{t}, J=7.5 \mathrm{~Hz}, \mathrm{CH}\left(\mathrm{CH}_{2} \mathrm{CH}_{3}\right)_{2}\right), 0.72\left(3 \mathrm{H}, \mathrm{t}, J=7.5 \mathrm{~Hz}, \mathrm{CH}\left(\mathrm{CH}_{2} \mathrm{CH}_{3}\right)_{2}\right), 0.85(4 \mathrm{H}, \mathrm{m}$, $\left.\mathrm{CH}\left(\mathrm{CH}_{2} \mathrm{CH}_{3}\right)_{2}\right), 0.98\left(3 \mathrm{H}, \mathrm{t}, J=7.5 \mathrm{~Hz}, \mathrm{CH}\left(\mathrm{CH}_{2} \mathrm{CH}_{3}\right)_{2}\right), 1.06\left(3 \mathrm{H}, \mathrm{t}, J=7.5 \mathrm{~Hz}, \mathrm{CH}\left(\mathrm{CH}_{2} \mathrm{CH}_{3}\right)_{2}\right), 1.23$ $\left(3 \mathrm{H}, \mathrm{d}, J=6.8 \mathrm{~Hz}, \mathrm{H}-2^{\prime}\right), 1.40(1 \mathrm{H}, \mathrm{m}, \mathrm{H}-4 \mathrm{~A}), 1.53\left(4 \mathrm{H}, \mathrm{m}, \mathrm{CH}\left(\mathrm{CH}_{2} \mathrm{CH}_{3}\right)_{2}\right), 1.65(2 \mathrm{H}, \mathrm{m}, \mathrm{H}-3), 1.75(1 \mathrm{H}$, m, H-4B), $2.15(1 \mathrm{H}, \mathrm{m}, \mathrm{H}-5), 2.74(1 \mathrm{H}, \mathrm{t}, J=10.1 \mathrm{~Hz}, \mathrm{H}-1), 3.40(1 \mathrm{H}, \mathrm{dd}, J=11.2$ and $5.5 \mathrm{~Hz}, \mathrm{H}-7), 3.75$ $(1 \mathrm{H}, \mathrm{m}, \mathrm{H}-2), 3.67\left(1 \mathrm{H}, \mathrm{d}, J=12.4 \mathrm{~Hz}, \mathrm{H}-1^{\prime \prime} \mathrm{A}\right), 3.71\left(1 \mathrm{H}, \mathrm{d}, J=12.4 \mathrm{~Hz}, \mathrm{H}-1^{\prime \prime} \mathrm{B}\right), 3.76\left(1 \mathrm{H}, \mathrm{m}, \mathrm{H}-1^{\prime}\right), 4.58$ $\left(1 \mathrm{H}\right.$, quintet, $\left.J=5.7 \mathrm{~Hz}, \mathrm{CH}\left(\mathrm{CH}_{2} \mathrm{CH}_{3}\right)_{2}\right), 4.73\left(1 \mathrm{H}\right.$, quintet, $\left.J=5.7 \mathrm{~Hz}, \mathrm{CH}\left(\mathrm{CH}_{2} \mathrm{CH}_{3}\right)_{2}\right), 7.1$ - $7.6(2 \mathrm{H}$, Ar-H). ${ }^{13} \mathrm{C}-\mathrm{NMR} \delta$ (ppm) $\left(\mathrm{CDCl}_{3}\right): 9.3\left(\mathrm{CH}_{3}, \mathrm{CH}\left(\mathrm{CH}_{2} \mathrm{CH}_{3}\right)_{2}\right), 9.5\left(\mathrm{CH}_{3}, \mathrm{CH}\left(\mathrm{CH}_{2} \mathrm{CH}_{3}\right)_{2}\right), 9.8\left(\mathrm{CH}_{3}\right.$, $\left.\mathrm{CH}\left(\mathrm{CH}_{2} \mathrm{CH}_{3}\right)_{2}\right), 9.9\left(\mathrm{CH}_{3}, \mathrm{CH}\left(\mathrm{CH}_{2} \mathrm{CH}_{3}\right)_{2}\right), 14.3\left(\mathrm{CH}_{3}, \mathrm{C}-2^{\prime}\right), 25.4\left(\mathrm{CH}_{2}, \mathrm{CH}\left(\mathrm{CH}_{2} \mathrm{CH}_{3}\right)_{2}\right), 25.8\left(\mathrm{CH}_{2}\right.$, $\left.\mathrm{CH}\left(\mathrm{CH}_{2} \mathrm{CH}_{3}\right)_{2}\right), 25.9\left(\mathrm{CH}_{2}, \mathrm{C}-3\right), 26.1\left(\mathrm{CH}_{2}, \mathrm{CH}\left(\mathrm{CH}_{2} \mathrm{CH}_{3}\right)_{2} \times 2\right), 26.2\left(\mathrm{CH}_{2}, \mathrm{C}-3\right), 28.5\left(\mathrm{CH}_{2}, \mathrm{C}-4\right), 41.2$ $(\mathrm{CH}, \mathrm{C}-5), 50.0\left(\mathrm{CH}, \mathrm{C}-1^{\prime \prime}\right), 52.6\left(\mathrm{CH}_{2}, \mathrm{C}-1\right), 54.7(\mathrm{CH}, \mathrm{C}-7), 57.2\left(\mathrm{CH}, \mathrm{C}-1^{\prime}\right), 61.8\left(\mathrm{CH}_{2}, \mathrm{C}-2\right), 77.9(\mathrm{CH}$, $\left.\mathrm{CH}\left(\mathrm{CH}_{2} \mathrm{CH}_{3}\right)_{2}\right), 78.6\left(\mathrm{CH}, \mathrm{CH}\left(\mathrm{CH}_{2} \mathrm{CH}_{3}\right)_{2}\right), 79.3\left(\mathrm{CH}, \mathrm{C}-1^{\prime \prime \prime}\right), 125.2-128.8(\mathrm{CHx} 20, \mathrm{Ar}), 141.3\left(\mathrm{C}, \mathrm{C}_{\mathrm{ipso}}\right)$, $144.0\left(\mathrm{C}, \mathrm{C}_{\mathrm{ipso}}\right), 144.2\left(\mathrm{C}, \mathrm{C}_{\mathrm{ipso}}\right), 148.2$ (C, $\left.\mathrm{C}_{\mathrm{ipso}}\right), 174.4$ (COOR, C-8), 174.7 (COOR, C-6).

When using 1,2-epoxycyclohexane, $160 \mathrm{mg}$ of crude product were obtained and chromatographed to obtain $86 \mathrm{mg}$ (yield $43 \%$ ) of $\mathbf{1 8}$ and $17 \mathrm{mg}$ (yield 10\%) of $\mathbf{1 6}$.

Pentan-3-yl (1R,2R,5R)-2-(benzyl((R)-1-phenylethyl)amino)-5-((R)-1-((1S,2R)-2-hydroxycyclohexyl)2-oxo-2-(pentan-3-yloxy)ethyl)cyclopentane-1-carboxylate (18): IR ( $\left.\mathrm{cm}^{-1}\right)$ : 3511, 2969, 1726, 1458, $1117,748,700 .{ }^{1} \mathrm{H}-\mathrm{NMR} \delta(\mathrm{ppm})\left(200 \mathrm{MHz}, \mathrm{CDCl}_{3}\right): 0.78-0.96\left(9 \mathrm{H}, \mathrm{m}, \mathrm{CH}\left(\mathrm{CH}_{2} \mathrm{CH}_{3}\right)_{2}\right), 1.11(3 \mathrm{H}, \mathrm{m}$, $\left.\mathrm{CH}\left(\mathrm{CH}_{2} \mathrm{CH}_{3}\right)_{2}\right), 1.29\left(3 \mathrm{H}, \mathrm{d}, J=6.8 \mathrm{~Hz}, \mathrm{H}-2^{\prime}\right), 1.42-1.93\left(8 \mathrm{H}, \mathrm{m}, \mathrm{CH}\left(\mathrm{CH}_{2} \mathrm{CH}_{3}\right)_{2} ; 2 \mathrm{H}, \mathrm{m}, \mathrm{H}-3 ; 2 \mathrm{H}, \mathrm{m}\right.$, $\left.\mathrm{H}-4 ; 1 \mathrm{H}, \mathrm{H}-1^{\prime \prime \prime}\right), 2.43(1 \mathrm{H}, \mathrm{dd}, \mathrm{H}-7), 2.94(1 \mathrm{H}, \mathrm{m}, \mathrm{H}-5), 3.04(1 \mathrm{H}, \mathrm{t}, J=7.1 \mathrm{~Hz}, \mathrm{H}-1), 3.61(1 \mathrm{H}, \mathrm{q}, J=7.7$ $\mathrm{Hz}, \mathrm{H}-2), 3.67\left(1 \mathrm{H}, \mathrm{d}, J=14.3, \mathrm{H}-1^{\prime \prime} \mathrm{A}\right), 3.87\left(1 \mathrm{H}, \mathrm{d}, J=14.3, \mathrm{H}-1^{\prime \prime} \mathrm{B}\right), 3.89\left(1 \mathrm{H}, \mathrm{q}, J=6.8 \mathrm{~Hz}, \mathrm{H}-1^{\prime}\right), 4.17$ $(1 \mathrm{H},-\mathrm{OH}), 4.75\left(2 \mathrm{H}, \mathrm{m}, \mathrm{CH}\left(\mathrm{CH}_{2} \mathrm{CH}_{3}\right)_{2} \times 2\right)$, 7.1-7.6 $(10 \mathrm{H}, \mathrm{Ar}-\mathrm{H})$.

When using ClCOOEt as electrophile, $275 \mathrm{mg}$ of crude product were obtained and chromatographed. The desired product was eluted in hexane/EtOAc 95:5 to furnish $80 \mathrm{mg}$ (yield 54\%) of 19.

1-Ethyl-3-(pentan-3-yl) 2-((1S,2R,3R)-3-(benzyl((R)-1-phenylethyl)amino)-2-((pentan-3-yloxy)carbonyl)cyclopentyl)malonate (19): IR $\left(\mathrm{cm}^{-1}\right)$ : 2972, 1732, 1462, 1113, 1030, 910, 748. H.R.M.S.: calcd for $\mathrm{C}_{36} \mathrm{H}_{51} \mathrm{NO}_{6}$ : 593.3716; found: 593.3793. ${ }^{1} \mathrm{H}-\mathrm{NMR} \delta(\mathrm{ppm})\left(400 \mathrm{MHz}, \mathrm{CDCl}_{3}\right): 0.82(3 \mathrm{H}, \mathrm{m}$, $\left.\mathrm{CH}\left(\mathrm{CH}_{2} \mathrm{CH}_{3}\right)_{2}\right), 0.84\left(3 \mathrm{H}, \mathrm{t}, J=7.5 \mathrm{~Hz}, \mathrm{CH}\left(\mathrm{CH}_{2} \mathrm{CH}_{3}\right)_{2} \times 2\right), 0.86\left(3 \mathrm{H}, \mathrm{m}, \mathrm{CH}\left(\mathrm{CH}_{2} \mathrm{CH}_{3}\right)_{2}\right), 1.21(3 \mathrm{H}$, $\left.\mathrm{t}, \mathrm{COOCH}_{2} \mathrm{CH}_{3}\right) 1.31\left(3 \mathrm{H}, \mathrm{d}, J=6.8 \mathrm{~Hz}, \mathrm{H}-2^{\prime}\right), 1.35-1.47\left(4 \mathrm{H}, \mathrm{m}, \mathrm{CH}\left(\mathrm{CH}_{2} \mathrm{CH}_{3}\right)_{2}\right), 1.52-1.58(4 \mathrm{H}, \mathrm{m}$, $\left.\mathrm{CH}\left(\mathrm{CH}_{2} \mathrm{CH}_{3}\right)_{2}\right) 1.55(1 \mathrm{H}, \mathrm{m}, \mathrm{H}-4 \mathrm{~A}), 1.75(2 \mathrm{H}, \mathrm{m}, \mathrm{H}-3), 1.87(1 \mathrm{H}, \mathrm{m}, \mathrm{H}-4 \mathrm{~B}), 2.68(1 \mathrm{H}, \mathrm{m}, \mathrm{H}-5), 2.62$ $(1 \mathrm{H}, \mathrm{d}, J=7.5, \mathrm{H}-7), 2.76(1 \mathrm{H}, \mathrm{t}, J=7 \mathrm{~Hz}, \mathrm{H}-1), 3.32(1 \mathrm{H}, \mathrm{d}, J=6 \mathrm{~Hz}, \mathrm{H}-7), 3.40(1 \mathrm{H}, \mathrm{d}, J=6 \mathrm{~Hz}$, $\mathrm{H}-7), 3.68\left(1 \mathrm{H}, \mathrm{d}, J=4.2 \mathrm{~Hz}, \mathrm{H}-1^{\prime \prime} \mathrm{A}\right), 3.71\left(1 \mathrm{H}, \mathrm{d}, J=4.2 \mathrm{~Hz}, \mathrm{H}-1^{\prime \prime} \mathrm{B}\right), 3.89\left(1 \mathrm{H}, \mathrm{q}, J=6.8 \mathrm{~Hz}, \mathrm{H}-1^{\prime}\right)$,

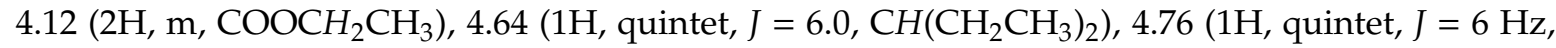
$\left.\mathrm{CH}\left(\mathrm{CH}_{2} \mathrm{CH}_{3}\right)_{2}\right)$, 7.1-7.6 (10H, Ar-H). ${ }^{13} \mathrm{C}-\mathrm{NMR} \delta(\mathrm{ppm})\left(\mathrm{CDCl}_{3}\right): 9.7\left(\mathrm{CH}_{3}, \mathrm{CH}\left(\mathrm{CH}_{2} \mathrm{CH}_{3}\right)_{2} \times 2\right), 9.8$ $\left(\mathrm{CH}_{3}, \mathrm{CH}\left(\mathrm{CH}_{2} \mathrm{CH}_{3}\right)_{2} \times 2\right), 15.4\left(\mathrm{CH}_{3}, \mathrm{C}-2^{\prime}\right), 14.3\left(\mathrm{CH}_{3}, \mathrm{COOCH}_{2} \mathrm{CH}_{3}\right), 15.6\left(\mathrm{CH}_{3}, \mathrm{C}-2^{\prime}\right), 25.6\left(\mathrm{CH}_{2}\right.$, $\left.\mathrm{CH}\left(\mathrm{CH}_{2} \mathrm{CH}_{3}\right)_{2} \times 2\right), 26.4\left(\mathrm{CH}_{2}, \mathrm{CH}\left(\mathrm{CH}_{2} \mathrm{CH}_{3}\right)_{2} \times 2\right), 27.8\left(\mathrm{CH}_{2}, \mathrm{C}-3\right), 27.9\left(\mathrm{CH}_{2}, \mathrm{C}-4\right), 27.5\left(\mathrm{CH}_{2}, \mathrm{C}-4\right)$, $41.1(\mathrm{CH}, \mathrm{C}-5), 50.2\left(\mathrm{CH}_{2}, \mathrm{C}-1^{\prime \prime}\right), 50.3\left(\mathrm{CH}_{2}, \mathrm{C}-1^{\prime \prime}\right), 52.8(\mathrm{CH}, \mathrm{C}-1), 53.0(\mathrm{CH}, \mathrm{C}-1), 54.3\left(\mathrm{CH}_{2}, \mathrm{C}-7\right)$, $55.0\left(\mathrm{CH}_{2}, \mathrm{C}-7\right), 57.7\left(\mathrm{CH}, \mathrm{C}-1^{\prime}\right), 61.3\left(\mathrm{CH}_{2}, \mathrm{COOCH}_{2} \mathrm{CH}_{3}\right), 64.5(\mathrm{CH}, \mathrm{C}-2), 64.8(\mathrm{CH}, \mathrm{C}-2), 76.6(\mathrm{CH}$, 
$\left.\mathrm{CH}\left(\mathrm{CH}_{2} \mathrm{CH}_{3}\right)_{2}\right), 76.8\left(\mathrm{CH}, \mathrm{CH}\left(\mathrm{CH}_{2} \mathrm{CH}_{3}\right)_{2}\right), 77.9\left(\mathrm{CH}, \mathrm{CH}\left(\mathrm{CH}_{2} \mathrm{CH}_{3}\right)_{2}\right), 78.3\left(\mathrm{CH}, \mathrm{CH}\left(\mathrm{CH}_{2} \mathrm{CH}_{3}\right)_{2}\right), 124.4$ $\left(\mathrm{CH} 2,{\left.\mathrm{C}-1^{\prime \prime}\right)}^{\prime \prime}, 126.1\right.$ - 128.8 (CHx10, Ar), 141.8 (C, $\left.\mathrm{C}_{i p s o}\right), 141.8$ (C, $\left.\mathrm{C}_{i p s o}\right), 144.4$ (C, C-7), 168.7 (COOR, C-8), 174.6 (COOR, C-6).

\subsection{Synthesis of Nepetalactone Derivative $\mathbf{2 6}$}

We started the synthesis similarly to the preparation of $\mathbf{1 4}$, so $49 \mathrm{mg}$ of this compound were obtained. Then, to a solution of $\mathbf{1 4}$ in benzene $(3.5 \mathrm{~mL}), \mathrm{NaOMe}(4.8 \mathrm{mg}, 0.089 \mathrm{mmol})$ was added and the reaction mixture heated up to reflux for $4 \mathrm{~h}$. Then, the mixture was extracted with ether and washed with $\mathrm{H}_{2} \mathrm{O}$ and brine. After being dried with $\mathrm{Na}_{2} \mathrm{SO}_{4}$ and filtered, the solvent was evaporated and $48 \mathrm{mg}$ (yield 100\%) of $\mathbf{2 0}$ were thus obtained. A quantitative amount of $\mathbf{2 0}$ was also obtained when treating 14 (47 mg) with $\mathrm{NaH}(2.5 \mathrm{mg}, 0.103 \mathrm{mmol})$ under an Ar atmosphere for $6 \mathrm{~h}$. Then, the reaction mixture was washed with $\mathrm{H}_{2} \mathrm{O}$ and dissolved in ether. After being dried with $\mathrm{Na}_{2} \mathrm{SO}_{4}$, filtered and the solvent evaporated, $46 \mathrm{mg}$ of 20 (100\% yield) were obtained.

Pentan-3-yl (1R,2R,5S)-2-(benzyl((R)-1-phenylethyl)amino)-5-(3-oxo-3-(pentan-3-yloxy)prop-1-en2-yl)-cyclopentane-1-carboxylate (20): IR (cm-1): 2969, 2940, 2880, 1724, 1495, 1464, 1373, 1271, 1161, $1117,1028,943,746,698 .{ }^{1} \mathrm{H}-\mathrm{NMR} \delta(\mathrm{ppm})\left(400 \mathrm{MHz}, \mathrm{CDCl}_{3}\right): 0.71\left(3 \mathrm{H}, \mathrm{t}, J=\mathrm{Hz}, \mathrm{CH}\left(\mathrm{CH}_{2} \mathrm{CH}_{3}\right)_{2}\right)$, $0.83\left(6 \mathrm{H}, \mathrm{m}, \mathrm{CH}\left(\mathrm{CH}_{2} \mathrm{CH}_{3}\right)_{2}\right), 0.90\left(3 \mathrm{H}, \mathrm{t}, J=\mathrm{Hz}, \mathrm{CH}\left(\mathrm{CH}_{2} \mathrm{CH}_{3}\right)_{2}\right), 1.30\left(3 \mathrm{H}, \mathrm{d}, J=\mathrm{Hz}, \mathrm{NCHCH}_{3}\right), 1.54$ $(4 \mathrm{H}, \mathrm{m}, \mathrm{H}-3, \mathrm{H}-4), 2.94\left(2 \mathrm{H}, \mathrm{m}, \mathrm{CH}\left(\mathrm{CH}_{2} \mathrm{CH}_{3}\right)_{2}\right), 3.03\left(2 \mathrm{H}, \mathrm{m}, \mathrm{CH}\left(\mathrm{CH}_{2} \mathrm{CH}_{3}\right)_{2}\right), 3.63\left(2 \mathrm{H}, \mathrm{m}, \mathrm{NCH}_{2} \mathrm{Ph}\right)$, $3.71\left(1 \mathrm{H}\right.$, quintet, $\left.J=\mathrm{Hz} \mathrm{NCHCH}_{3}\right), 4.57(1 \mathrm{H}, \mathrm{m}, \mathrm{H}-5), 4.79(1 \mathrm{H}, \mathrm{m}, \mathrm{H}-1), 5.53\left(1 \mathrm{H}, \mathrm{s}, \mathrm{CCH}_{2}-\mathrm{A}\right), 6.08$ $\left(1 \mathrm{H}, \mathrm{s}, \mathrm{CCH}_{2}-\mathrm{B}\right), 7.16-7.31(10 \mathrm{H}, \mathrm{Ar}) . \mathrm{RMN}{ }^{13} \mathrm{C} \delta(\mathrm{ppm})\left(\mathrm{CDCl}_{3}\right): 9.4\left(\mathrm{CH}_{3}, \mathrm{CH}\left(\mathrm{CH}_{2} \mathrm{CH}_{3}\right)_{2} \times 4,15.0\right.$ $\left(\mathrm{CH}_{3}, \mathrm{NCHCH}_{3}\right), 25.6\left(\mathrm{CH}_{2}, \mathrm{CH}\left(\mathrm{CH}_{2} \mathrm{CH}_{3}\right)_{2} \times 2\right), 26.6\left(\mathrm{CH}_{2}, \mathrm{CH}\left(\mathrm{CH}_{2} \mathrm{CH}_{3}\right)_{2} \times 2\right), 31.8\left(\mathrm{CH}_{2}, \mathrm{C}-3, \mathrm{C}-4\right)$, $45.4(\mathrm{CH}, \mathrm{C}-5), 50.2\left(\mathrm{CH}_{2}, \mathrm{NCH}_{2} \mathrm{Ph}\right), 53.5(\mathrm{CH}, \mathrm{C}-1), 57.7(\mathrm{CH}, \mathrm{NCH}), 63.6\left(\mathrm{CH}, \mathrm{NCHCH}_{3}\right), 77.9(\mathrm{CH}$, $\left.\mathrm{CH}\left(\mathrm{CH}_{2} \mathrm{CH}_{3}\right)_{2}\right), 124.4\left(\mathrm{CH}_{2}, \mathrm{CCH}_{2}\right), 141.7\left(\mathrm{C}_{\text {ipso }} \times 2\right), 144.4(\mathrm{C}, \mathrm{C}-7), 166.6(\mathrm{C}, \mathrm{COOR}), 174.5(\mathrm{C}, \mathrm{COOR})$.

To a solution of $14(38 \mathrm{mg}, 0.07 \mathrm{mmol})$ in benzene $(3 \mathrm{~mL}), \mathrm{HCl}$ gas was added at $0{ }^{\circ} \mathrm{C}$ and then, a catalytic amount of $p \mathrm{TsOH}$ was added and the reaction mixture was heated up to $60^{\circ} \mathrm{C}$ for $24 \mathrm{~h}$. Then, the resulting mixture was extracted with ether and washed with $\mathrm{NaHCO}_{3}$ saturated solution and $\mathrm{H}_{2} \mathrm{O}$. After being dried with $\mathrm{Na}_{2} \mathrm{SO}_{4}$, filtered and the solvent evaporated, the desired compound 21 was eluted with hexane/EtOAc 95:5. $7 \mathrm{mg}$ (yield 30\%) were obtained.

Pentan-3-yl (4S,4aS,7R)-7-(benzyl((R)-1-phenylethyl)amino)-1-oxooctahydrocyclopenta[c]pyran-4carboxylate (21): H.R.M.S.: calcd for $\mathrm{C}_{29} \mathrm{H}_{37} \mathrm{NO}_{4}$ : 479.3036; found: $363.2764 .{ }^{1} \mathrm{H}-\mathrm{NMR} \delta$ (ppm) (400 $\left.\mathrm{MHz}, \mathrm{CDCl}_{3}\right): 0.83\left(3 \mathrm{H}, \mathrm{t}, J=5.0 \mathrm{~Hz}, \mathrm{CH}\left(\mathrm{CH}_{2} \mathrm{CH}_{3}\right)_{2}\right), 0.86\left(3 \mathrm{H}, \mathrm{t}, J=5.0 \mathrm{~Hz}, \mathrm{CH}\left(\mathrm{CH}_{2} \mathrm{CH}_{3}\right)_{2}\right), 1.34(3 \mathrm{H}$, $\left.\mathrm{d}, J=6.9 \mathrm{~Hz}, \mathrm{H}-2^{\prime}\right), 1.51(1 \mathrm{H}, \mathrm{m}, \mathrm{H}-6 \mathrm{~A}), 1.53(1 \mathrm{H}, \mathrm{m}, \mathrm{H}-7 \mathrm{~A}), 1.55-1.79\left(4 \mathrm{H}, \mathrm{m}, \mathrm{CH}\left(\mathrm{CH}_{2} \mathrm{CH}_{3}\right)_{2}\right), 1.79(1 \mathrm{H}$, m, H-6B), 1.82 (1H, m, H-7B), $2.74(1 \mathrm{H}, \mathrm{dd}, J=11.6$ and $4.6 \mathrm{~Hz}, \mathrm{H}-9), 2.94(1 \mathrm{H}, \mathrm{m}, \mathrm{H}-5), 3.07$ (1H, ddd, $J=9.8,9.8$ and $7.5 \mathrm{~Hz}, \mathrm{H}-4), 3.73(1 \mathrm{H}, \mathrm{m}, \mathrm{H}-8), 3.76\left(1 \mathrm{H}, \mathrm{d}, J=15.1 \mathrm{~Hz}, \mathrm{H}-1^{\prime \prime} \mathrm{A}\right), 3.85\left(1 \mathrm{H}, \mathrm{m}, \mathrm{H}-1^{\prime}\right), 3.89$ $\left(1 \mathrm{H}, \mathrm{d}, J=15.1 \mathrm{~Hz}, \mathrm{H}-1^{\prime \prime} \mathrm{B}\right), 4.11(1 \mathrm{H}, \mathrm{dd}, \mathrm{J}=17.9$ and $7.5 \mathrm{~Hz}, \mathrm{H}-3 \mathrm{~B}), 4.30(1 \mathrm{H}, \mathrm{dd}, J=11.7$ and $9.8 \mathrm{~Hz}$, $\mathrm{H}-3 \mathrm{~A}), 4.74\left(1 \mathrm{H}\right.$, quintet, $\left.J=6 \mathrm{~Hz}, \mathrm{CH}\left(\mathrm{CH}_{2} \mathrm{CH}_{3}\right)_{2}\right)$ 7.1-7.6 $(10 \mathrm{H}, \mathrm{Ar}-\mathrm{H}) .{ }^{13} \mathrm{C}-\mathrm{NMR} \delta(\mathrm{ppm})\left(\mathrm{CDCl}_{3}\right): 9.5$ $\left(\mathrm{CH}_{3}, \mathrm{CH}\left(\mathrm{CH}_{2} \mathrm{CH}_{3}\right)_{2} \times 2\right), 17.3\left(\mathrm{CH}_{3}, \mathrm{C}-2^{\prime}\right), 25.9\left(\mathrm{CH}_{2}, \mathrm{CH}\left(\mathrm{CH}_{2} \mathrm{CH}_{3}\right)_{2} \times 2\right), 27.3\left(\mathrm{CH}_{2}, \mathrm{C}-7\right), 27.8\left(\mathrm{CH}_{2}\right.$, C-6), 40.0 (CH, C-5), 11540.8 (CH, C-4), $46.3(\mathrm{CH}, \mathrm{C}-8), 49.8\left(\mathrm{CH}_{2}, \mathrm{C}-1^{\prime \prime}\right), 58.2\left(\mathrm{CH}, \mathrm{C}-1^{\prime}\right), 59.8(\mathrm{CH}$, C-9), $67.3\left(\mathrm{CH}_{2}, \mathrm{C}-3\right), 77.9\left(\mathrm{CH}, \mathrm{CH}\left(\mathrm{CH}_{2} \mathrm{CH}_{3}\right)_{2}\right), 126.4-128.1(10 \mathrm{C}, \mathrm{Ar}), 142.8\left(\mathrm{C}, \mathrm{C}_{i p s o}\right), 144.8\left(\mathrm{C}, \mathrm{C}_{i p s o}\right)$, 170.8 (C, C-1), 173.9 (COOR, C-11).

Also, to a solution of $14(85 \mathrm{mg})$ in $\mathrm{CH}_{2} \mathrm{Cl}_{2}(4 \mathrm{~mL}), m \mathrm{CPBA}(66 \mathrm{mg}, 0.385 \mathrm{mmol})$ was added. After $6 \mathrm{~h}$ of stirring, saturated $\mathrm{Na}_{2} \mathrm{~S}_{2} \mathrm{O}_{3}$ solution $(4 \mathrm{~mL})$ was added and the resulting mixture was eluted with $\mathrm{CH}_{2} \mathrm{Cl}_{2}$ and washed with $\mathrm{H}_{2} \mathrm{O}, \mathrm{NaHCO}_{3}$ and saturated $\mathrm{Na}_{2} \mathrm{~S}_{2} \mathrm{O}_{3}$ solution. The resulting mixture was dried with $\mathrm{Na}_{2} \mathrm{SO}_{4}$ and, after being filtered, it was chromatographed. The desired compound was eluted with hexane/EtOAc 95:5 to give $31 \mathrm{mg}$ (yield 82\%) of 22. Also, when the reaction was performed in one pot, starting from compound 2 and adding $(R)-\mathbf{1}, \mathrm{HCHO}$ and then, directly at the reaction mixture, $m$ CPBA was added, both 22 (yield 69\%) and 23 (yield 6\%) were obtained.

Pentan-3-yl (R)-5-((S)-3-hydroxy-1-oxo-1-(pentan-3-yloxy)propan-2-yl)cyclopent-1-ene-1-carboxylate (22): $[\alpha]_{\mathrm{D}}{ }^{26}=+9.6\left(\mathrm{CHCl}_{3}, \mathrm{c}=1.00\right)$. IR $\left(\mathrm{cm}^{-1}\right): 3524,2880,1709,1098,920$. H.R.M.S.: calcd for $\mathrm{C}_{19} \mathrm{H}_{32} \mathrm{O}_{5}$ : 340.2250; found: 340.2218. ${ }^{1} \mathrm{H}-\mathrm{NMR} \delta(\mathrm{ppm})\left(400 \mathrm{MHz}, \mathrm{CDCl}_{3}\right): 0.90(12 \mathrm{H}, \mathrm{m}$, 
$\left.\mathrm{CH}\left(\mathrm{CH}_{2} \mathrm{CH}_{3}\right)_{2}\right), 1.59\left(8 \mathrm{H}, \mathrm{m}, \mathrm{CH}\left(\mathrm{CH}_{2} \mathrm{CH}_{3}\right)_{2}\right), 1.88(1 \mathrm{H}, \mathrm{m}, \mathrm{H}-4 \mathrm{~B}), 2.08(1 \mathrm{H}, \mathrm{m}, \mathrm{H}-4 \mathrm{~A}), 2.44(1 \mathrm{H}, \mathrm{m}$, H-3B), 2.49 (1H, m, H-3A), $3.29(1 \mathrm{H}, \mathrm{q}, J=4.4 \mathrm{~Hz}, \mathrm{H}-7), 3.57\left(1 \mathrm{H}, \mathrm{dd}, J=11.6\right.$ and $4.1 \mathrm{~Hz}, \mathrm{H}-1^{\prime \prime \prime}$ ), $3.62(1 \mathrm{H}, \mathrm{m}, \mathrm{H}-5), 3.80\left(1 \mathrm{H}, \mathrm{dd}, J=11.6\right.$ and $\left.8.1 \mathrm{~Hz}, \mathrm{H}-1^{\prime \prime \prime} \mathrm{B}\right), 4.83\left(2 \mathrm{H}, \mathrm{q}, J=6.0 \mathrm{~Hz}, \mathrm{CH}\left(\mathrm{CH}_{2} \mathrm{CH}_{3}\right)_{2}\right)$, $6.86(1 \mathrm{H}, \mathrm{dd}, J=2.4 \mathrm{~Hz}, \mathrm{H}-2) .{ }^{13} \mathrm{C}-\mathrm{NMR} \delta(\mathrm{ppm})\left(\mathrm{CHCl}_{3}\right): 10.1\left(\mathrm{CH}_{3}, \mathrm{CH}\left(\mathrm{CH}_{2} \mathrm{CH}_{3}\right)_{2} \times 4\right), 26.8\left(\mathrm{CH}_{2}\right.$, $\left.\mathrm{CH}\left(\mathrm{CH}_{2} \mathrm{CH}_{3}\right)_{2} \times 2\right), 26.9\left(\mathrm{CH}_{2}, \mathrm{CH}\left(\mathrm{CH}_{2} \mathrm{CH}_{3}\right)_{2} \times 2\right), 27.0\left(\mathrm{CH}_{2}, \mathrm{C}-4\right), 32.8\left(\mathrm{CH}_{2}, \mathrm{C}-3\right), 44.5(\mathrm{CH}, \mathrm{C}-5), 49.4$ $(\mathrm{CH}, \mathrm{C}-7), 60.3\left(\mathrm{CH} 2, \mathrm{C}-1^{\prime \prime \prime}\right), 76.9\left(\mathrm{CH}, \mathrm{CH}\left(\mathrm{CH}_{2} \mathrm{CH}_{3}\right)_{2}\right), 77.6\left(\mathrm{CH}, \mathrm{CH}\left(\mathrm{CH}_{2} \mathrm{CH}_{3}\right)_{2}\right), 137.2(\mathrm{C}, \mathrm{C}-1), 146.1$ (C, C-2), 165.3 (C, C-6), 175.1 (C, C-8).

Pentan-3-yl (S)-5-((S)-3-hydroxy-1-oxo-1-(pentan-3-yloxy)propan-2-yl)cyclopent-1-ene-1-carboxylate (23): IR $\left(\mathrm{cm}^{-1}\right)$ : 3493, 2969, 2880, 1711, 1632, 1462, 1383, 1267, 1202, 1101, 1047, 934, 752. ${ }^{1} \mathrm{H}-\mathrm{NMR} \delta$ (ppm) (400 MHz, $\left.\mathrm{CDCl}_{3}\right): 0.90\left(12 \mathrm{H}, \mathrm{m}, \mathrm{CH}\left(\mathrm{CH}_{2} \mathrm{CH}_{3}\right)_{2}\right), 1.51\left(8 \mathrm{H}, \mathrm{m}, \mathrm{CH}\left(\mathrm{CH}_{2} \mathrm{CH}_{3}\right)_{2}\right), 1.85(1 \mathrm{H}, \mathrm{m}, \mathrm{H}-4 \mathrm{~A})$, $2.15(1 \mathrm{H}, \mathrm{m}, \mathrm{H}-4 \mathrm{~B}), 2.44(1 \mathrm{H}, \mathrm{m}, \mathrm{H}-3 \mathrm{~A}), 2.62(1 \mathrm{H}, \mathrm{m}, \mathrm{H}-3 \mathrm{~B}), 3.05(1 \mathrm{H}, \mathrm{q}, J=4.4 \mathrm{~Hz}, \mathrm{H}-7), 3.33(1 \mathrm{H}, \mathrm{dd}$, $J=11.6$ and $\left.4.1 \mathrm{~Hz}, \mathrm{H}-1^{\prime \prime \prime} \mathrm{A}\right), 3.75(1 \mathrm{H}, \mathrm{m}, \mathrm{H}-5), 3.79\left(1 \mathrm{H}, \mathrm{dd}, J=11.6\right.$ and $\left.8.1 \mathrm{~Hz}, \mathrm{H}-1^{\prime \prime \prime} \mathrm{B}\right), 4.76(2 \mathrm{H}$, quintet, $\left.J=6.0 \mathrm{~Hz}, \mathrm{CH}\left(\mathrm{CH}_{2} \mathrm{CH}_{3}\right)_{2} \times 2\right), 6.80(1 \mathrm{H}, \mathrm{m}, \mathrm{H}-2) .{ }^{13} \mathrm{C}-\mathrm{NMR} \delta(\mathrm{ppm})\left(\mathrm{CHCl}_{3}\right): 9.82\left(\mathrm{CH}_{3}\right.$, $\left.\mathrm{CH}\left(\mathrm{CH}_{2} \mathrm{CH}_{3}\right)_{2} \times 4\right), 26.53\left(\mathrm{CH}_{2}, \mathrm{CH}\left(\mathrm{CH}_{2} \mathrm{CH}_{3}\right)_{2} \times 4\right), 27.9\left(\mathrm{CH}_{2}, \mathrm{C}-4\right), 31.8\left(\mathrm{CH}_{2}, \mathrm{C}-3\right), 43.3(\mathrm{CH}, \mathrm{C}-5), 50.3$ $(\mathrm{CH}, \mathrm{C}-7), 63.1\left(\mathrm{CH} 2, \mathrm{C}-1^{\prime \prime \prime}\right), 77.0\left(\mathrm{CH}, \mathrm{CH}\left(\mathrm{CH}_{2} \mathrm{CH}_{3}\right)_{2}\right), 77.5\left(\mathrm{CH}, \mathrm{CH}\left(\mathrm{CH}_{2} \mathrm{CH} 3\right)_{2}\right), 138.2(\mathrm{C}, \mathrm{C}-1), 145.2$ (C, C-2), 165.6 (C, C-6), 173.9 (C, C-8).

To a solution of $23(8.5 \mathrm{mg}, 0.025 \mathrm{mmol})$ in benzene $(3 \mathrm{~mL})$, a catalytic amount of $p \mathrm{TsOH}$ was added and the reaction mixture was heated up to $60^{\circ} \mathrm{C}$ for $24 \mathrm{~h}$, then, it was extracted with ether and washed with saturated $\mathrm{NaHCO}_{3}$ solution and $\mathrm{H}_{2} \mathrm{O}$. The resulting mixture was dried with $\mathrm{Na}_{2} \mathrm{SO}_{4}$, filtered and the solvent evaporated. After chromatography, $5 \mathrm{mg}$ (yield 65\%) of the desired compound 24 were eluted with hexane/EtOAc 9:1.

Pentan-3-yl (4S,4aR)-1-oxo-1,3,4,4a,5,6-hexahydrocyclopenta[c]pyran-4-carboxylate (24): IR ( $\left.\mathrm{cm}^{-1}\right)$ : $3445,2969,1728,1636,1464,1402,1256,1157,1092,1057,910,733 .{ }^{1} \mathrm{H}-\mathrm{NMR} \delta$ (ppm) $\left(400 \mathrm{MHz}, \mathrm{CDCl}_{3}\right)$ : $0.87\left(6 \mathrm{H}, \mathrm{m}, \mathrm{CH}\left(\mathrm{CH}_{2} \mathrm{CH}_{3}\right)_{2} \times 2\right), 1.55-1.62\left(4 \mathrm{H}, \mathrm{m}, \mathrm{CH}\left(\mathrm{CH}_{2} \mathrm{CH}_{3}\right)_{2}\right), 1.78(1 \mathrm{H}, \mathrm{m}, \mathrm{H}-6 \mathrm{~B}), 2.31(1 \mathrm{H}, \mathrm{m}$, H-6A), $2.48(2 \mathrm{H}, \mathrm{m}, \mathrm{H}-7), 2.69(1 \mathrm{H}, \mathrm{ddd}, J=11.5,11.5$ and $3.1 \mathrm{~Hz}, \mathrm{H}-4), 3.19(1 \mathrm{H}, \mathrm{m}, \mathrm{H}-5), 4.32(1 \mathrm{H}$, $\mathrm{dd}, J=11.9$ and $3.5 \mathrm{~Hz}, \mathrm{H}-3 \mathrm{~A}), 4.52(1 \mathrm{H}, \mathrm{dd}, J=11.9$ and $2.9 \mathrm{~Hz}, \mathrm{H}-3 \mathrm{~B}), 4.80(1 \mathrm{H}$, quintet, $J=6 \mathrm{~Hz}$ $\left.\mathrm{CH}\left(\mathrm{CH}_{2} \mathrm{CH}_{3}\right)_{2}\right), 7.07(1 \mathrm{H}, \mathrm{m}, \mathrm{H}-8) . \mathrm{RMN}^{13} \mathrm{C} \delta(\mathrm{ppm})\left(\mathrm{CDCl}_{3}\right): 9.5\left(\mathrm{CH}_{3}, \mathrm{CH}\left(\mathrm{CH}_{2} \mathrm{CH}_{3}\right)_{2} \mathrm{x} 2\right), 26.3\left(\mathrm{CH}_{2}\right.$, $\left.\mathrm{CH}\left(\mathrm{CH}_{2} \mathrm{CH}_{3}\right)_{2} \times 2\right), 31.7\left(\mathrm{CH}_{2}, \mathrm{C}-6\right), 31.9\left(\mathrm{CH}_{2}, \mathrm{C}-7\right), 45.0(\mathrm{CH}, \mathrm{C}-4), 47.0(\mathrm{CH}, \mathrm{C}-5), 69.6\left(\mathrm{CH}_{2}, \mathrm{C}-3\right), 77.9$ $\left(\mathrm{CH}, \mathrm{CH}\left(\mathrm{CH}_{2} \mathrm{CH}_{3}\right)_{2}\right), 132.4$ (C, C-9), 147.4 (C, C-8), 162.3 (C, C-1), 170.2 (COOR, C-11).

To a solution of $22(13 \mathrm{mg}, 0.038 \mathrm{mmol})$ in benzene $(3 \mathrm{~mL})$, a catalytic amount of $p \mathrm{TsOH}$ was added and the reaction flask was heated to $60{ }^{\circ} \mathrm{C}$ for $24 \mathrm{~h}$. Then, the reaction mixture was diluted with ether and washed with saturated $\mathrm{NaHCO}_{3}$ solution and $\mathrm{H}_{2} \mathrm{O}$. The resulting mixture was dried with $\mathrm{Na}_{2} \mathrm{SO}_{4}$ and, after being filtered and the solvent evaporated, it was chromatographed and the desired compound was eluted with hexane/EtOAc 9:1 to give $6 \mathrm{mg}$ (yield 77\%) of 25 .

Pentan-3-yl (4S,4aR)-1-oxo-1,3,4,4a,5,6-hexahydrocyclopenta[c]pyran-4-carboxylate (25): $[\alpha]_{\mathrm{D}}{ }^{26}=-0.56$ $\left(\mathrm{CHCl}_{3}, \mathrm{c}=0.89\right)$. IR $\left(\mathrm{cm}^{-1}\right)$ : 2969, 1728, 1256, 1157, 910, 733. H.R.M.S.: calcd for $\mathrm{C}_{14} \mathrm{H}_{20} \mathrm{O}_{4}$ : 252.3062 ; found: $252.2386 . \mathrm{RMN}^{1} \mathrm{H} \delta$ (ppm) $\left(400 \mathrm{MHz}, \mathrm{CDCl}_{3}\right): 0.84\left(3 \mathrm{H}, \mathrm{t}, J=7.4 \mathrm{~Hz}, \mathrm{CH}\left(\mathrm{CH}_{2} \mathrm{CH}_{3}\right)_{2}\right), 0.87$ $\left(3 \mathrm{H}, \mathrm{t}, J=7.4 \mathrm{~Hz}, \mathrm{CH}\left(\mathrm{CH}_{2} \mathrm{CH}_{3}\right)_{2}\right), 1.53-1.60\left(4 \mathrm{H}, \mathrm{m}, \mathrm{CH}\left(\mathrm{CH}_{2} \mathrm{CH}_{3}\right)_{2}\right), 1.91(1 \mathrm{H}, \mathrm{m}, \mathrm{H}-6 \mathrm{~A}), 2.31(1 \mathrm{H}, \mathrm{m}$, H-6B), $2.51(1 \mathrm{H}, \mathrm{m}, \mathrm{H}-7 \mathrm{~A}), 2.51(1 \mathrm{H}, \mathrm{m}, \mathrm{H}-7 \mathrm{~B}), 3.02(1 \mathrm{H}, \mathrm{ddd}, J=11.5,11.5$ and $3.1 \mathrm{~Hz}, \mathrm{H}-4), 3.31(1 \mathrm{H}, \mathrm{m}$, $\mathrm{H}-5), 4.35(1 \mathrm{H}, \mathrm{dd}, J=11.9$ and $3.5 \mathrm{~Hz}, \mathrm{H}-3 \mathrm{~A}), 4.55(1 \mathrm{H}, \mathrm{dd}, J=11.9$ and $2.9 \mathrm{~Hz}, \mathrm{H}-3 \mathrm{~B}), 4.80(1 \mathrm{H}$, quintet, $\left.J=6 \mathrm{~Hz} \mathrm{CH}\left(\mathrm{CH}_{2} \mathrm{CH}_{3}\right)_{2}\right), 6.98(1 \mathrm{H}, \mathrm{m}, \mathrm{H}-8) . \mathrm{RMN}^{13} \mathrm{C} \delta(\mathrm{ppm})\left(\mathrm{CDCl}_{3}\right)$ : $9.2\left(\mathrm{CH}_{3}, \mathrm{CH}\left(\mathrm{CH}_{2} \mathrm{CH}_{3}\right)_{2}\right), 9.8$ $\left(\mathrm{CH}_{3}, \mathrm{CH}\left(\mathrm{CH}_{2} \mathrm{CH}_{3}\right)_{2}\right), 26.4\left(\mathrm{CH}_{2}, \mathrm{CH}\left(\mathrm{CH}_{2} \mathrm{CH}_{3}\right)_{2 \times}\right.$ 2), $29.3\left(\mathrm{CH}_{2}, \mathrm{C}-6\right), 31.9\left(\mathrm{CH}_{2}, \mathrm{C}-7\right), 43.1(\mathrm{CH}, \mathrm{C}-4)$, $43.8(\mathrm{CH}, \mathrm{C}-5), 69.8\left(\mathrm{CH}_{2}, \mathrm{C}-3\right), 78.2\left(\mathrm{CH}, \mathrm{CH}\left(\mathrm{CH}_{2} \mathrm{CH}_{3}\right)_{2}\right), 132.7(\mathrm{C}, \mathrm{C}-9), 145.3(\mathrm{C}, \mathrm{C}-8), 163.2(\mathrm{C}, \mathrm{C}-1)$, 170 (COOR, C-11).

Finally, when it comes to the syntheses of nepetalactone derivative 26, first, a lithium dimethyl-cuprate solution was prepared by adding MeLi $1.6 \mathrm{M}(0.13 \mathrm{~mL}, 0.21 \mathrm{mmol})$ to a solution of $\mathrm{CuI}(20 \mathrm{mg}, 0.103 \mathrm{mmol})$ in ether $(2.50 \mathrm{~mL})$ at $0{ }^{\circ} \mathrm{C}$. Then, $25(13 \mathrm{mg}, 0.05 \mathrm{mmol})$ in ether $(1.50 \mathrm{~mL})$ was added and, after $90 \mathrm{~min}$ of stirring, $3.00 \mathrm{~mL}$ of $\mathrm{NH}_{4} \mathrm{Cl}$ saturated solution were added. The resulting reaction mixture was diluted with ether and dried with $\mathrm{Na}_{2} \mathrm{SO}_{4}$. After being filtered, $11 \mathrm{mg}$ of crude 
product were obtained and chromatographed. The desired nepetalactone was eluted in hexane/EtOAc 95:5 To afford $10 \mathrm{mg}$ (yield 80\%) of $\mathbf{2 6}$.

Pentan-3-yl (4S,4aS,7S,7aR)-7-methyl-1-oxooctahydrocyclopenta[c]pyran-4-carboxylate (26): $\mathrm{IR}\left(\mathrm{cm}^{-1}\right)$ : 2967, 1736, 1196, 1034, 909. H.R.M.S.: calcd for $\mathrm{C}_{15} \mathrm{H}_{24} \mathrm{O}_{4}$ : 268.1675; found: 269.1768. ${ }^{1} \mathrm{H}-\mathrm{NMR} \delta$ (ppm) $\left(400 \mathrm{MHz}, \mathrm{CDCl}_{3}\right): 0.88\left(3 \mathrm{H}, \mathrm{t}, J=5.0 \mathrm{~Hz}, \mathrm{CH}\left(\mathrm{CH}_{2} \mathrm{CH}_{3}\right)_{2}\right), 0.89\left(3 \mathrm{H}, \mathrm{t}, J=5.0 \mathrm{~Hz}, \mathrm{CH}\left(\mathrm{CH}_{2} \mathrm{CH}_{3}\right)_{2}\right), 1.20$ $(3 \mathrm{H}, \mathrm{d}, J=6.4 \mathrm{~Hz}, \mathrm{H}-10), 1.50(1 \mathrm{H}, \mathrm{m}, \mathrm{H}-6 \mathrm{~A}), 1.54-1.60\left(4 \mathrm{H}, \mathrm{m}, \mathrm{CH}\left(\mathrm{CH}_{2} \mathrm{CH}_{3}\right)_{2}\right), 1.88(1 \mathrm{H}, \mathrm{m}, \mathrm{H}-6 \mathrm{~B}), 1.98$ $(1 \mathrm{H}, \mathrm{m}, \mathrm{H}-7 \mathrm{~A}), 2.07(1 \mathrm{H}, \mathrm{m}, \mathrm{H}-8), 2.26(1 \mathrm{H}, \mathrm{m}, \mathrm{H}-7 \mathrm{~B}), 2.51(1 \mathrm{H}, \mathrm{dd}, J=10.5$ and $10.3 \mathrm{~Hz}, \mathrm{H}-9), 2.94(1 \mathrm{H}$, m, H-5), $3.06(1 \mathrm{H}, \mathrm{ddd}, J=10.4,6.1$ and $4.6 \mathrm{~Hz}, \mathrm{H}-4), 4.37(1 \mathrm{H}, \mathrm{m}, \mathrm{H}-3 \mathrm{~A}), 4.41(1 \mathrm{H}, \mathrm{m}, 1.5 \mathrm{~Hz}, \mathrm{H}-3 \mathrm{~B})$, $4.78\left(1 \mathrm{H}\right.$, quintet, $\left.J=6 \mathrm{~Hz}, \mathrm{CH}\left(\mathrm{CH}_{2} \mathrm{CH}_{3}\right)_{2}\right) .{ }^{13} \mathrm{C}-\mathrm{NMR} \delta(\mathrm{ppm})\left(\mathrm{CDCl}_{3}\right): 9.8\left(\mathrm{CH}_{3}, \mathrm{CH}\left(\mathrm{CH}_{2} \mathrm{CH}_{3}\right)_{2} \times 2\right)$, $19.0\left(\mathrm{CH}_{3}, \mathrm{C}-10\right), 26.4\left(\mathrm{CH}_{2}, \mathrm{CH}\left(\mathrm{CH}_{2} \mathrm{CH}_{3}\right)_{2} \times 2\right), 28.1\left(\mathrm{CH}_{2}, \mathrm{C}-7\right), 35.3\left(\mathrm{CH}_{2}, \mathrm{C}-6\right), 37.4(\mathrm{CH}, \mathrm{C}-8), 42.1$ $(\mathrm{CH}, \mathrm{C}-5), 42.7(\mathrm{CH}, \mathrm{C}-4), 50.5(\mathrm{CH}, \mathrm{C}-9), 64.7(\mathrm{CH} 2, \mathrm{C}-3), 78.0\left(\mathrm{CH}, \mathrm{CH}\left(\mathrm{CH}_{2} \mathrm{CH}_{3}\right)_{2}\right), 170.4(\mathrm{C}, \mathrm{C}-1)$, 173.2 (COOR, C-11).

Supplementary Materials: The following are available online. Characterization data, IR, ${ }^{1} \mathrm{H} N M R,{ }^{13} \mathrm{C} N M R$ and HRMS Spectra of products and HMQC, HMBC, COSY and ROESY, where it has been carried out.

Author Contributions: Conceptualization, N.M.G., methodology, N.M.G. and D.D., experiments, A.M. and V.E.R., writing-original draft preparation, N.M.G., writing-review and editing, D.D. and A.M. All authors have read and agreed to the published version of the manuscript.

Funding: We are indebted to European Regional Development Fund (FEDER), Spanish Ministerio de Economía y Competitividad (MINECO) for its support (CTQ 2015-68175-R), Junta de Castilla y León (UIC 21), and the Universidad de Salamanca. A.M.C. thanks European Social Fund (FSE) and USAL for his grant.

Acknowledgments: The authors thank also for support from Servicios de la Universidad de Salamanca (Nucleus): A.M. Lithgow for the NMR spectra and César Raposo for the mass spectra.

Conflicts of Interest: The authors declare no conflict of interest.

\section{References}

1. Dinda, B.; Debnath, S.; Harigaya, Y. Naturally Occurring Secoiridoids and Bioactivity of Naturally Occurring Iridoids and Secoiridoids. A Review, Part 2. Chem. Pharm. Bull. 2007, 55, 689-728. [CrossRef]

2. Dinda, B.; Roy Chowdhury, D.; Mohanta, B.C. Naturally Occurring Iridoids, Secoiridoids and Their Bioactivity. An Updated Review, Part 3. Chem. Pharm. Bull. 2009, 57, 765-796. [CrossRef]

3. Dinda, B.; Debnath, S.; Harigaya, Y. Naturally Occurring Iridoids. A Review, Part 1. Chem. Pharm. Bull. 2007, 55, 159-222. [CrossRef] [PubMed]

4. Xiong, J.; Wu, X.Y.; Wang, P.P.; Lau, C.; Fan, H.; Ma, G.L.; Tang, Y.; Li, J.; Hu, J.F. Acylated Iridoid Diglycosides from the Cultivated Endangered Ornamental Tree Gmelina Hainanensis. Phytochem. Lett. 2018, 25, 17-21. [CrossRef]

5. Lee, D.H.; Shin, J.S.; Kang, S.Y.; Lee, S.B.; Lee, J.S.; Ryu, S.M.; Lee, K.T.; Lee, D.; Jang, D.S. Iridoids from the Roots of Patrinia Scabra and Their Inhibitory Potential on LPS-Induced Nitric Oxide Production. J. Nat. Prod. 2018, 81, 1468-1473. [CrossRef] [PubMed]

6. $\quad$ Lee, D.H.; Shin, J.S.; Lee, J.S.; Kang, S.Y.; Han, H.S.; Ryu, S.M.; Lee, K.T.; Lee, D.; Jang, D.S. Non-Glycosidic Iridoids from the Roots of Patrinia Scabra and Their Nitric Oxide Production Inhibitory Effects. Arch. Pharm. Res. 2019, 42, 766-772. [CrossRef]

7. Fu, N.; Yang, Z.-L.; Pauchet, Y.; Paetz, C.; Brandt, W.; Boland, W.; Burse, A. A Cytochrome P450 from the Mustard Leaf Beetles Hydroxylates Geraniol, a Key Step in Iridoid Biosynthesis. Insect Biochem. Mol. Biol. 2019, 113, 103212. [CrossRef]

8. Vedachalam, S.; Murugesh, N.; Chakraborty, P.; Karvembu, R.; Liu, X.W. NHC Catalyzed Enantioselective Coates-Claisen Rearrangement: A Rapid Access to the Dihydropyran Core for Oleuropein Based Secoiridoids. New J. Chem. 2018, 42, 1832-1839. [CrossRef]

9. Morikawa, T.; Xie, H.; Pan, Y.; Ninomiya, K.; Yuan, D.; Jia, X.; Yoshikawa, M.; Nakamura, S.; Matsuda, H.; Muraoka, O. A Review of Biologically Active Natural Products from a Desert Plant Cistanche Tubulosa. Chem. Pharm. Bull. (Tokyo) 2019, 67, 675-689. [CrossRef] 
10. Win, N.N.; Kodama, T.; Lae, K.Z.W.; Win, Y.Y.; Ngwe, H.; Abe, I.; Morita, H. Bis-Iridoid and Iridoid Glycosides: Viral Protein R Inhibitors from Picrorhiza Kurroa Collected in Myanmar. Fitoterapia 2019, 134, 101-107. [CrossRef]

11. Luca, S.V.; Miron, A.; Ignatova, S.; Skalicka-Woźniak, K. An Overview of the Two-Phase Solvent Systems Used in the Countercurrent Separation of Phenylethanoid Glycosides and Iridoids and Their Biological Relevance. Phytochem. Rev. 2019, 18, 377-403. [CrossRef]

12. Pang, X.; Zhao, J.-y.; Yu, H.-y.; Yu, L.-y.; Wang, T.; Zhang, Y.; Gao, X.-m.; Han, L.-f. Secoiridoid Analogues from the Fruits of Ligustrum Lucidum and Their Inhibitory Activities against Influenza A Virus. Bioorganic Med. Chem. Lett. 2018, 28, 1516-1519. [CrossRef] [PubMed]

13. Dimitrova, P.; Alipieva, K.; Grozdanova, T.; Simova, S.; Bankova, V.; Georgiev, M.I.; Popova, M.P. New Iridoids from Verbascum Nobile and Their Effect on Lectin-Induced T Cell Activation and Proliferation. Food Chem. Toxicol. 2018, 111, 605-615. [CrossRef] [PubMed]

14. de Souza, A.d.S.L.; Peret, A.C.; Hamoy, M.; de Souza, R.A.L.; Torres, M.F.; Barbas, L.A.L. Propofol and Essential Oil of Nepeta Cataria Induce Anaesthesia and Marked Myorelaxation in Tambaqui Colossoma Macropomum: Implications on Cardiorespiratory Responses. Aquaculture 2019, 500, 160-169. [CrossRef]

15. Boros, C.A.; Stermitz, F.R. Iridoids. An Updated Review, Part II. J. Nat. Prod. 1991, 54, 1173-1246. [CrossRef]

16. Aydin, S.; Beis, R.; Öztürk, Y.; Hüsnü, K.; Baser, C. Nepetalactone: A New Opioid Analgesic from Nepeta Caesarea Boiss. J. Pharm. Pharmacol. 1998, 50, 813-817. [CrossRef]

17. Valimehr, S.; Sanjarian, F.; Sabouni, F.; Hashemi, H.; Sharafi, A. Anti-Inflammatory Effects of Essential Oil, Aerial Parts and Hairy Roots Extracts of Nepeta Pogonosperma on Rat Brain Mixed Cells. Res. J. Pharmacogn. 2015, 2, 5-10.

18. Salehi, B.; Valussi, M.; Jugran, A.K.; Martorell, M.; Ramírez-Alarcón, K.; Stojanović-Radić, Z.Z.; Antolak, H.; Kręgiel, D.; Mileski, K.S.; Sharifi-Rad, M.; et al. Nepeta Species: From Farm to Food Applications and Phytotherapy. Trends Food Sci. Technol. 2018, 80, 104-122. [CrossRef]

19. Süntar, I.; Nabavi, S.M.; Barreca, D.; Fischer, N.; Efferth, T. Pharmacological and Chemical Features of Nepeta, L. Genus: Its Importance as a Therapeutic Agent. Phyther. Res. 2018, 32, 185-198. [CrossRef]

20. Calixto, J.B.; Beirith, A.; Ferreira, J.; Santos, A.R.S.; Filho, V.C.; Yunes, R.A. Naturally Occurring Antinociceptive Substances from Plants. Phyther. Res. 2000, 14, 401-418. [CrossRef]

21. Lenardão, E.J.; Savegnago, L.; Jacob, R.G.; Victoria, F.N.; Martinez, D.M. Antinociceptive Effect of Essential Oils and Their Constituents: An Update Review. J. Braz. Chem. Soc. 2016, 27, 435-474. [CrossRef]

22. Birkett, M.A.; Hassanali, A.; Hoglund, S.; Pettersson, J.; Pickett, J.A. Repellent Activity of Catmint, Nepeta Cataria, and Iridoid Nepetalactone Isomers against Afro-Tropical Mosquitoes, Ixodid Ticks and Red Poultry Mites. Phytochemistry 2011, 72, 109-114. [CrossRef] [PubMed]

23. Takenaka, Y.; Tanahashi, T.; Shintaku, M.; Sakai, T.; Nagakura, N.; Parida. Secoiridoid Glucosides from Fraxinus Americana. Phytochemistry 2000, 55, 275-284. [CrossRef]

24. Wei, X.; Xie, H.; Ge, X.; Zhang, F. Iridoids from Dunnia Sinensis. Phytochemistry 2000, 53, 837-840. [CrossRef]

25. Candish, L.; Lupton, D.W. The Total Synthesis of (-)-7-Deoxyloganin via N-Heterocyclic Carbene Catalyzed Rearrangement of $\alpha, \beta$-Unsaturated Enol Esters. Org. Lett. 2010, 12, 4836-4839. [CrossRef] [PubMed]

26. Davies, S.G.; Smith, A.D.; Price, P.D. The Conjugate Addition of Enantiomerically Pure Lithium Amides as Homochiral Ammonia Equivalents: Scope, Limitations and Synthetic Applications. Tetrahedron Asymmetry 2005, 16, 2833-2891. [CrossRef]

27. Davies, S.G.; Fletcher, A.M.; Roberts, P.M.; Thomson, J.E. The Conjugate Addition of Enantiomerically Pure Lithium Amides as Chiral Ammonia Equivalents Part II: 2005-2011. Tetrahedron Asymmetry 2012, 23, 1111-1153. [CrossRef]

28. Davies, S.G.; Fletcher, A.M.; Roberts, P.M.; Thomson, J.E. The Conjugate Addition of Enantiomerically Pure Lithium Amides as Chiral Ammonia Equivalents Part III: 2012-2017. Tetrahedron Asymmetry 2017, 28, 1842-1868. [CrossRef]

29. Nieto, C.T.; Eames, J.; Garrido, N.M. 8.1.6 Product Subclass 6: Lithium Amides. Sci. Synth. Knowl. Updates 2019, 1, 66. [CrossRef] 
30. Urones, J.G.; Garrido, N.M.; Díez, D.; Dominguez, S.H.; Davies, S.G. Conjugate Addition to $(\alpha, \beta)(\alpha, \beta)$-Diendioate Esters by Lithium ( $\alpha$ - Methylbenzyl)Benzylamide: Tandem Addition-Cyclisation versus Double Addition. Tetrahedron Asymmetry 1999, 10, 1637-1641. [CrossRef]

31. Rubia, A.G.; Salgado, M.M.; Nieto, C.T.; Manchado, A.; Díez, D.; Sanz, F.; Garrido, N.M. Asymmetric Synthesis of Tert-Butyl ((1R,4aR,8R,8aR)-1-Hydroxyoctahydro-1H-Isochromen-8-Yl)Carbamate. Tetrahedron Asymmetry 2017, 28, 1394-1400. [CrossRef]

32. Nieto, C.T.; González-Núñez, V.; Rodríguez, R.E.; Diez, D.; Garrido, N.M. Design, Synthesis, Pharmacological Evaluation and Molecular Dynamics of $\beta$-Amino Acids Morphan-Derivatives as Novel Ligands for Opioid Receptors. Eur. J. Med. Chem. 2015, 101, 150-162. [CrossRef] [PubMed]

33. Garrido, N.M.; Díez, D.; Domínguez, S.H.; García, M.; Sánchez, M.R.; Davies, S.G. Asymmetric Synthesis of Pent-3-Yl (R)-6-Methyl-Cyclohex-1-Ene Carboxylate. Tetrahedron Asymmetry 2006, 17, 2183-2186. [CrossRef]

34. Garrido, N.M.; Nieto, C.T.; Diez, D. Enantioselective Synthesis of a $(1 R, 5 R, 9 R)-2-A z a b i c y c l o$ [3.3.1]Nonane-9-Carboxylic Acid with an Embedded Morphan Motif: A Multipurpose Product. Synlett 2013, 24, 169-172. [CrossRef]

35. Urones, J.G.; Garrido, N.M.; Díez, D.; Domínguez, S.H.; Davies, S.G. Asymmetric Synthesis of (R)- and (S)-Methyl (2-Methoxy-Carbonylcyclopent-2-Enyl)Acetate and (R)- and (S)-2-(2-HydroxyMethyl-Cyclopent-2-Enyl)Ethanol. Tetrahedron: Asymmetry 1997, 8, 2683-2685. [CrossRef]

36. Urones, J.G.; Garrido, N.M.; Díez, D.; El Hammoumi, M.M.; Dominguez, S.H.; Casaseca, J.A.; Davies, S.G.; Smith, A.D. Asymmetric Synthesis of the Stereoisomers of 2-Amino-5-CarboxymethylCyclopentane-1-Carboxylate. Org. Biomol. Chem. 2004, 2, 364-372. [CrossRef]

37. Garrido, N.M.; El Hammoumi, M.M.; Díez, D.; García, M.; Urones, J.G. A Novel Strategy towards the Asymmetric Synthesis of Orthogonally Funtionalised 2-N-Benzyl-N- $\alpha$-MethylbenzylAmino-5-Carboxymethyl- Cyclopentane-1-Carboxylic Acid. Molecules 2004, 9, 373-382. [CrossRef]

38. Garrido, N.M.; Sánchez, M.R.; Díez, D.; Sanz, F.; Urones, J.G. Enantioselective Synthesis of Cis-(2S,3R)and Trans-(2S,3S)- Piperidinedicarboxylic Acids Using Domino: Allylic Acetate and Ireland-Claisen Rearrangements and Michael Addition as the Key Steps. Tetrahedron Asymmetry 2011, 22, 872-880. [CrossRef]

39. Garrido, N.M.; Garcia, M.; Sánchez, M.R.; Diez, D.; Urones, J.G. Enantioselective Synthesis of (+)-L-733,060 and (+)-CP-99,994: Application of an Ireland-Claisen Rearrangement/Michael Addition Domino Sequence. Synlett 2010, 2010, 387-390. [CrossRef]

40. Garrido, N.M.; García, M.; Díez, D.; Sánchez, M.R.; Sanz, F.; Urones, J.G. Diastereoselective Synthesis of $\delta$-Aminoacids through Domino Ireland-Claisen Rearrangement and Michael Addition. Org. Lett. 2008, 10, 1687-1690. [CrossRef]

41. Salgado, M.M.; Manchado, A.; Nieto, C.T.; Díez, D.; Garrido, N.M. Asymmetric Synthesis of 2,3,6-Trisubstituted Piperidines via Baylis-Hillman Adducts and Lithium Amide through Domino Reaction. Synlett 2019, 30 A-E, 1-5. [CrossRef]

42. Manchado, A.; García, M.; Salgado, M.M.; Díez, D.; Garrido, N.M. A Novel Barton Decarboxylation Produces a 1,4-Phenyl Radical Rearrangement Domino Reaction. Tetrahedron 2018, 74, 5240-5247. [CrossRef]

43. Nieto, C.T.; Díez, D.; Garrido, N.M. To Be or Not to Be Butterfly: New Mechanistic Insights in the Aza-Michael Asymmetric Addition of Lithium (R)-N-Benzyl-N-( $\alpha$-Methylbenzyl)Amide. J. Comput. Chem. 2014, 35, 1846-1853. [CrossRef] [PubMed]

44. Costello, J.F.; Davies, S.G.; Ichihara, O. Origins of the High Stereoselectivity in the Conjugate Addition of Lithium( $\alpha$-Methylbenzyl)Benzylamide to t-Butyl Cinnamate. Tetrahedron: Asymmetry 1994, 5, 1999-2008. [CrossRef]

45. Davies, S.G.; Garrido, N.M.; Ichihara, O.; Iain, A.S.W. Asymmetric Syntheses of $\beta$-Phenylalanine, $\alpha$-Methyl- $\beta$-Phenylalanines and Derivatives. J. Chem. Soc., Chem. Commun. 1993, 8, 1153-1155. [CrossRef]

46. Taylor, R.J.K. Organocopper Conjugate Addition-Enolate Trapping Reactions. Synth. (Stuttg). 1985, 1985, 364-392. [CrossRef]

47. Sakan, T.; Murai, F.; Isoe, S.; Hayash, S.B. The Biologically Active C9-, C10-, and C11-Terpenes from Actinidia Polygama Miq., Boschniakia Rossica Hult, and Menyanthes Trifoliata, L. J. Chem. Soc. Jpn. Pure Chem. Sect. 1969, 90, 507-528. [CrossRef] 
48. Ohta, H.; Kobori, T.; Fujisawa, T. Regiospecific Introduction of Two Carbon Moieties into the Vicinal Positions of Cyclopentadiene and Synthesis of C9-Terpene Lactones. J. Org. Chem. 1977, 42, 1231-1235. [CrossRef]

Sample Availability: Samples of the compounds are available from the authors. article distributed under the terms and conditions of the Creative Commons Attribution (CC BY) license (http://creativecommons.org/licenses/by/4.0/). 